University of Tennessee Health Science Center UTHSC Digital Commons

\title{
$5-2008$
}

\section{Golgi Specificity and Development of Autoreactive B Cells}

\author{
Fazlullah Salar Khan Nawazi \\ University of Tennessee Health Science Center
}

Follow this and additional works at: https://dc.uthsc.edu/dissertations

Part of the Cells Commons

\section{Recommended Citation}

Nawazi, Fazlullah Salar Khan , "Golgi Specificity and Development of Autoreactive B Cells" (2008). Theses and Dissertations (ETD). Paper 176. http://dx.doi.org/10.21007/etd.cghs.2008.0224.

This Dissertation is brought to you for free and open access by the College of Graduate Health Sciences at UTHSC Digital Commons. It has been accepted for inclusion in Theses and Dissertations (ETD) by an authorized administrator of UTHSC Digital Commons. For more information, please contact jwelch30@uthsc.edu. 


\title{
Golgi Specificity and Development of Autoreactive B Cells
}

\begin{abstract}
$B$ cell receptors with certain heavy/light chain pairs predominate in the periphery of anti-DNA heavy chain transgenic mice while certain other B cell receptors are absent. We wanted to know why. To answer this, we re-constructed $B$ cell receptors represented in the anti-DNA transgenic mice by cloning them as single chain variable fragments (scFv) to analyze their affinity to dsDNA. scFv representing receptors that were recovered from the spleens of transgenic mice had very little to no affinity to dsDNA. scFv representing receptors that were absent in the spleen had high affinity to dsDNA. We therefore concluded that receptors with low or no affinity to DNA successfully passed central tolerance in the bone marrow while receptors that had high affinity to DNA were likely altered by receptor editing or deleted. We also encountered $B$ cell receptors that did not fit this conclusion. These were receptors that had anti-DNA heavy chains paired with the light chain Vk38c. They were an exception because, though they were present in the spleen, they bound DNA very well. How did these anti-DNA B cells escape central tolerance? In our effort to answer this puzzle, we discovered that receptors with the Vk38c light chain may have specificity to a Golgi antigen in addition to DNA. This suggested that such anti-DNA receptors may bind to an intracellular Golgi antigen and remain hidden inside the cell. Thus B cell receptor-cloistering in immature $B$ cells, during development in the bone marrow, may allow the escape of anti-DNA B cells from central tolerance. After escaping from the bone marrow, these $B$ cells become sequestered in the marginal zone of the spleen. Splenic B cells when immortalized as hybridomas show B cell receptor (IgM) accumulation in the Golgi. Receptor accumulates in the Golgi only when the heavy chain is paired with the Vk38c light chain. The location of $B$ cell receptor accumulation in these hybridomas ranges from the cis-Golgi to medial- and trans-Golgi with the bulk of the receptor being concentrated in the medial-Golgi. Fragmenting the Golgi architecture with nocodazole, caused the intracellular IgM accumulation to disperse to the same locations as the Golgi, further confirming the location. A novelty about these B cells is that they have an uncommon way of antibody secretion. Instead of secreting IgM pentamers as B cells normally do, these cells extrude IgM as aggregates of up to $2 \mu \mathrm{m}$. We named the aggregates "spherons" because of their appearance. The spherons do not have a membrane and are likely composed of the $\mathrm{m}$ heavy chain and the $\mathrm{k}$ light chain. In addition to extruding spherons, the B cells secrete soluble IgM as monomers and hemimers possibly as a consequence of IgM retention in the Golgi. Spherons have a high similarity to immune complexes deposited in kidneys of mice suffering from lupus nephritis. Spherons may be relevant in autoimmune nephritis and immunoglobulin deposition diseases. Understanding the mechanism of production of spherons could help design treatment strategies for patients suffering from autoimmune disease.
\end{abstract}

Document Type

Dissertation

Degree Name

Doctor of Philosophy (PhD)

\section{Program}

Molecular Sciences

Research Advisor

Marko Z. Radic, Ph.D.

\section{Keywords}

B cells, Golgi, Autoreactivity, IgM secretion 


\section{Subject Categories}

Cells | Medicine and Health Sciences

This dissertation is available at UTHSC Digital Commons: https://dc.uthsc.edu/dissertations/176 


\title{
GOLGI SPECIFICITY AND DEVELOPMENT OF
} AUTOREACTIVE B CELLS

\author{
A Dissertation \\ Presented for \\ The Graduate Studies Council \\ The University of Tennessee \\ Health Science Center \\ In Partial Fulfillment \\ Of the Requirements for the Degree \\ Doctor of Philosophy \\ From The University of Tennessee
}

By

Fazlullah Salar Khan Nawazi

May 2008 
Chapter 2 (C) 2008 by Proceedings of the National Academy of Sciences, USA All other material (C) 2008 by Fazlullah Salar Khan Nawazi

All rights reserved 


\section{DEDICATION}

This dissertation is dedicated to my parents

for the endless support they have provided me throughout my career. 


\section{ACKNOWLEDGEMENTS}

I would like to thank my mentor Dr.Marko Z Radic for his enormous support, guidance, and encouragement during my studies. His clear thinking, critical analysis, perseverance, and optimism will always be a huge influence on me. Next I would like to thank my committee members, Dr.Linda Hendershot, Dr.Monica Jablonski, Dr.John Cox, Dr.Elizabeth Fitzpatrick and Dr.Martha Howe for their suggestions and comments to improve many areas during my Ph.D. I appreciate and thank my committee members for taking their time from their busy schedules for me. I wish to thank Linda for her generosity in allowing me to use her labs' resources for a major part of my work. I thank Drs. Nina Luning Prak, Esther Witsch, Marvin Fritzler and Martin Weigert with whom we have had productive collaborations. I wish to thank Tim Higgins, Sue Farmer and Janie Van Prooijen as their help in the department made life a lot easier. I would like to thank my excellent friend and colleague Amy Cline for her tremendous help and goodwill in my personal and professional life and for making the lab a delightful work place. I would also like to thank my colleagues Indira, Nishanth, and Mahsa for their help and support. I would like to thank all the members in Linda's lab for their help during my time at St Jude. I am greatly indebted to all my friends and would like to offer thanks in particular to JP, Jeetu, Kishore and Karthik for their support during my Ph.D. I would like to thank my sister for all her support during my studies. Lastly, I believe that my parent's blessings, my wife's sacrifices and affection had held me together and made all this possible. 


\begin{abstract}
B cell receptors with certain heavy/light chain pairs predominate in the periphery of anti-DNA heavy chain transgenic mice while certain other B cell receptors are absent. We wanted to know why. To answer this, we re-constructed B cell receptors represented in the anti-DNA transgenic mice by cloning them as single chain variable fragments $(\mathrm{scFv})$ to analyze their affinity to dsDNA. scFv representing receptors that were recovered from the spleens of transgenic mice had very little to no affinity to dsDNA. $\mathrm{scFv}$ representing receptors that were absent in the spleen had high affinity to dsDNA. We therefore concluded that receptors with low or no affinity to DNA successfully passed central tolerance in the bone marrow while receptors that had high affinity to DNA were likely altered by receptor editing or deleted. We also encountered B cell receptors that did not fit this conclusion. These were receptors that had anti-DNA heavy chains paired with the light chain Vк38c. They were an exception because, though they were present in the spleen, they bound DNA very well. How did these anti-DNA B cells escape central tolerance? In our effort to answer this puzzle, we discovered that receptors with the Vк38c light chain may have specificity to a Golgi antigen in addition to DNA. This suggested that such anti-DNA receptors may bind to an intracellular Golgi antigen and remain hidden inside the cell. Thus B cell receptor-cloistering in immature B cells, during development in the bone marrow, may allow the escape of anti-DNA B cells from central tolerance. After escaping from the bone marrow, these B cells become sequestered in the marginal zone of the spleen. Splenic B cells when immortalized as hybridomas show B cell receptor (IgM) accumulation in the Golgi. Receptor accumulates in the Golgi only when the heavy chain is paired with the $\mathrm{V} \kappa 38 \mathrm{c}$ light chain. The location of $\mathrm{B}$ cell receptor accumulation in these hybridomas ranges from the cis-Golgi to medialand trans-Golgi with the bulk of the receptor being concentrated in the medial-Golgi. Fragmenting the Golgi architecture with nocodazole, caused the intracellular IgM accumulation to disperse to the same locations as the Golgi, further confirming the location. A novelty about these B cells is that they have an uncommon way of antibody secretion. Instead of secreting IgM pentamers as B cells normally do, these cells extrude IgM as aggregates of up to $2 \mu \mathrm{m}$. We named the aggregates "spherons" because of their appearance. The spherons do not have a membrane and are likely composed of the $\mu$ heavy chain and the $\kappa$ light chain. In addition to extruding spherons, the B cells secrete soluble IgM as monomers and hemimers possibly as a consequence of $\operatorname{IgM}$ retention in the Golgi. Spherons have a high similarity to immune complexes deposited in kidneys of mice suffering from lupus nephritis. Spherons may be relevant in autoimmune nephritis and immunoglobulin deposition diseases. Understanding the mechanism of production of spherons could help design treatment strategies for patients suffering from autoimmune disease.
\end{abstract}




\section{TABLE OF CONTENTS}

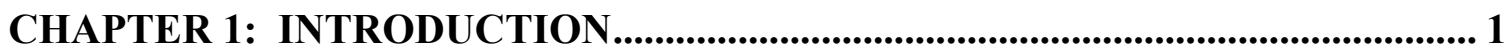

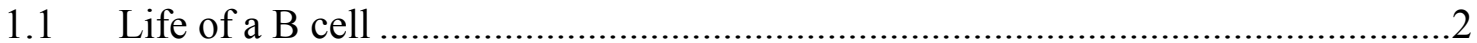

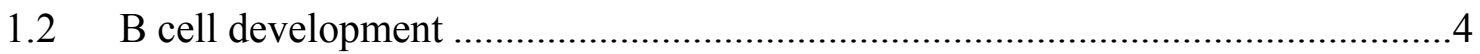

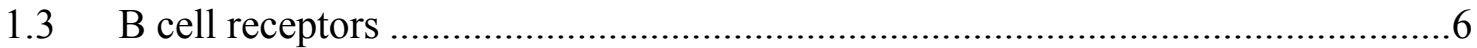

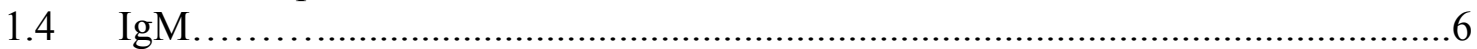

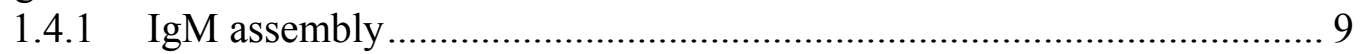

1.4.2 Importance of J chain for assembly into polymers .............................. 10

1.4.3 Glycosylation of IgM H chains........................................................ 10

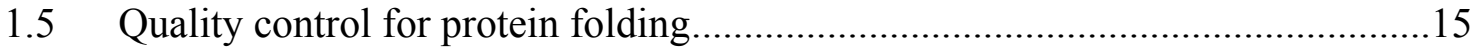

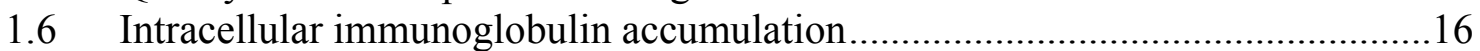

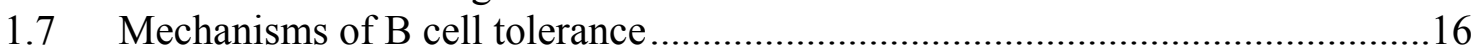

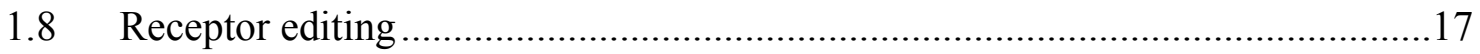

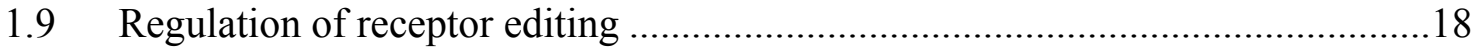

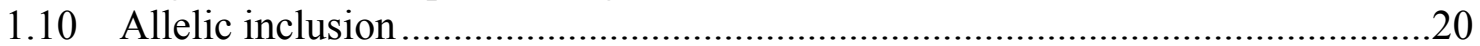

1.11 BCR signaling threshold in B cell development and tolerance induction ...........21

1.12 B cells may be positively selected …………................................................23

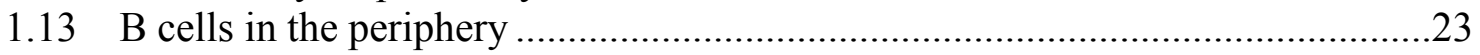

1.14 Check points to prevent autoreactivity ………..........................................24

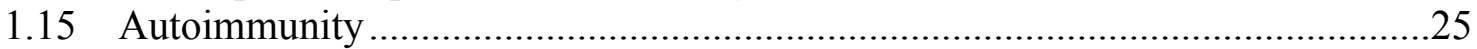

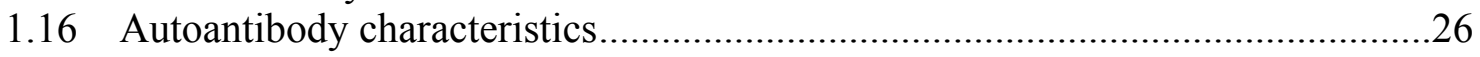

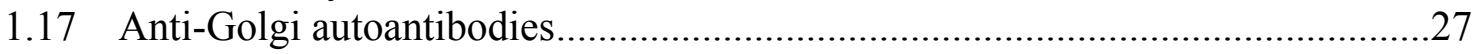

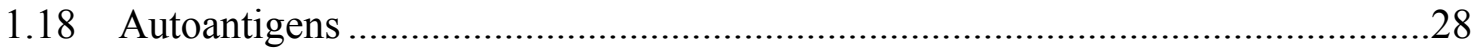

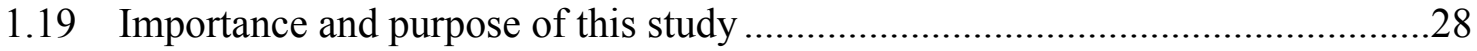

\section{CHAPTER 2: EDITING AND ESCAPE FROM EDITING IN ANTI-DNA B}

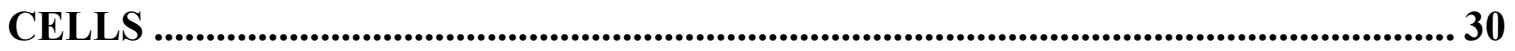

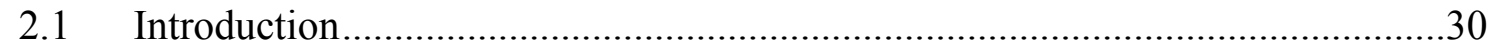

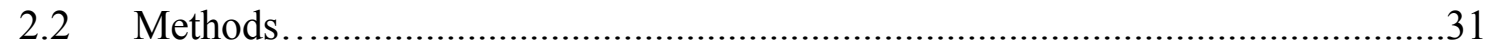

2.2.1 Construction of bivalent variable fragments ........................................ 31

2.2.2 Bacterial expression and purification of $\mathrm{scFv}$........................................ 31

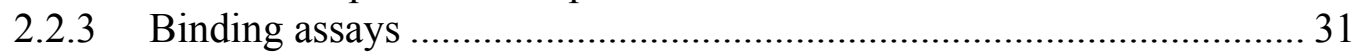

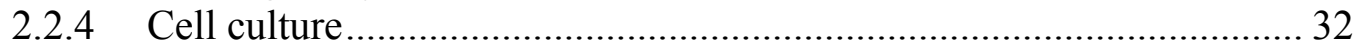

2.2.5 Immunofluorescence and confocal microscopy................................... 32

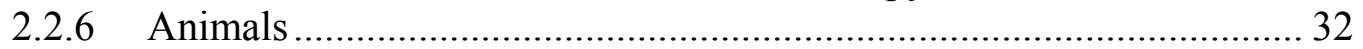

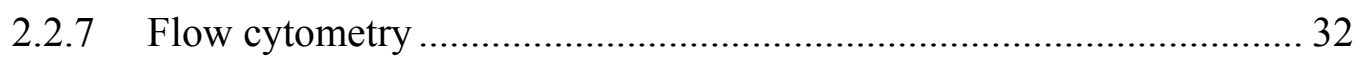

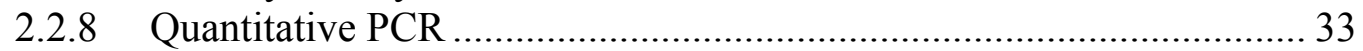

2.2.9 Single cell sorting, cDNA synthesis and PCR .................................... 33

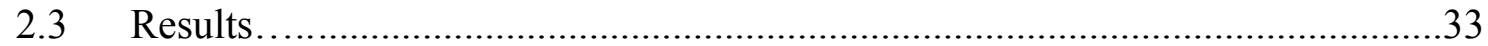

2.3.1 Analysis of autoreactivity ...................................................................... 33

2.3.2 DNA and PS binding of $\mathrm{V} \kappa 38 \mathrm{c}$ combinations......................................... 36

2.3.3 Specificity of $\mathrm{VH} / \mathrm{VL}$ pairs for intracellular antigens............................ 38

2.3.4 Distribution of Ig in hybridomas........................................................ 38

2.3.5 Cell surface expression of VH56R+Vк38c ……………………….... 43 
2.3.6 VH56R $+\mathrm{V} \kappa 38 \mathrm{c}$ receptors are enriched in marginal zone B cells......... 43

2.4 Discussion

\section{CHAPTER 3: EXTRUSION OF IgM POLYMER AGGREGATES "SPHERONS" AND SECRETION OF IgM HEMIMERS FROM B CELL} HYBRIDOMAS THAT ACCUMULATE IgM IN THE GOLGI ................................5 51

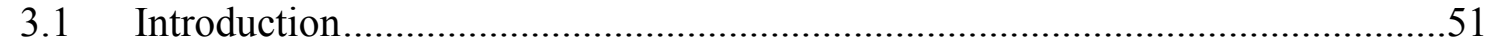

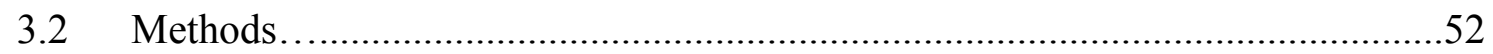

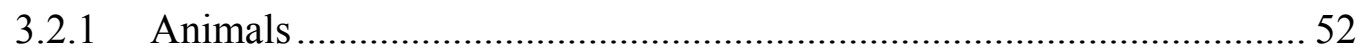

3.2.2 Cell culture ............................................................................ 52

3.2.3 Immunofluorescence and confocal microscopy................................ 52

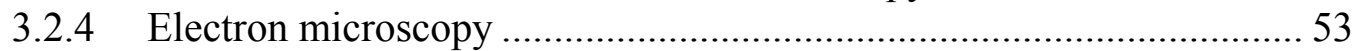

3.2.5 Metabolic labeling and analysis of cell fractions.............................. 53

3.2.6 Pulse and chase ......................................................................... 54

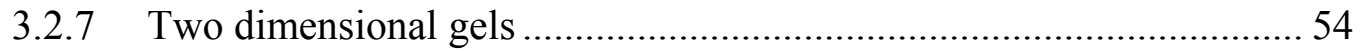

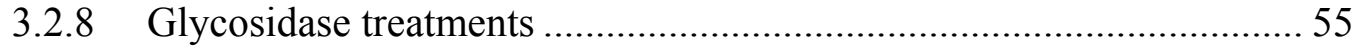

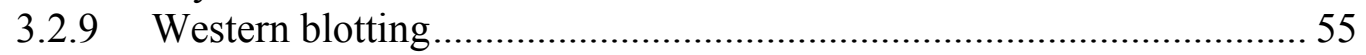

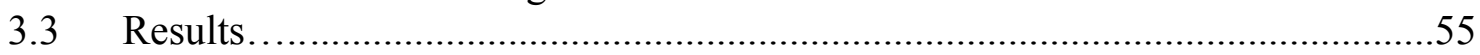

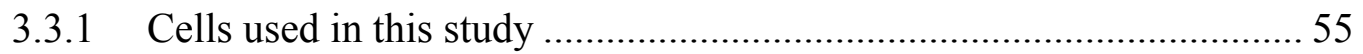

3.3.2 The VH56R+Vк38c IgM accumulates in the Golgi ........................... 56

3.3.3 Distribution and separation of IgM aggregates from the cell .............. 61

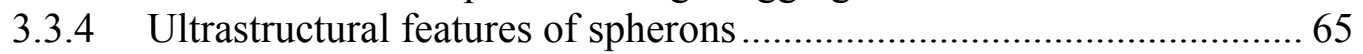

3.3.5 Characteristics of spheron production by H160.3 hybridoma .............. 65

3.3.6 Analysis of IgM under non-reducing conditions ..............................6 68

3.3.7 IgM fractions analyzed under reducing conditions............................6 68

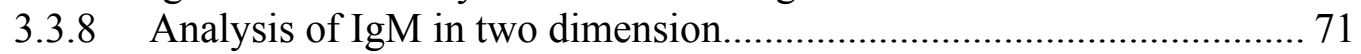

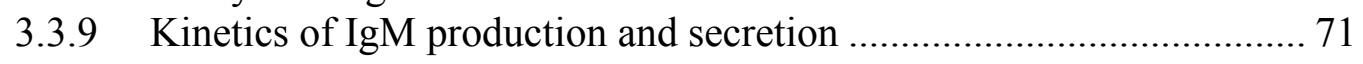

3.3.10 Hybridomas differ in glycosylation patterns.................................... 74

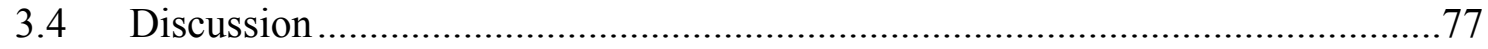

CHAPTER 4: GENERAL DISCUSSION ............................................................... 83

4.1 Our search for rules of receptor editing uncovers an exception ..........................83

4.2 Our efforts to identify the mechanism for escape of VH56R+Vk38c cells.........83

4.3 Determining the location of $\mathrm{VH} 56 \mathrm{R}+\mathrm{V} \kappa 38 \mathrm{c}$ accumulation ................................84

4.4 Our proposed mechanism for the production of hemimers and spherons ...........86

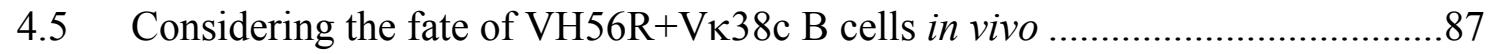

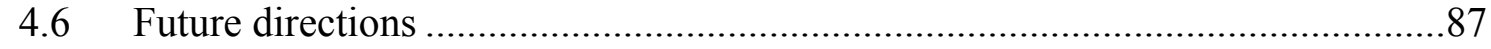

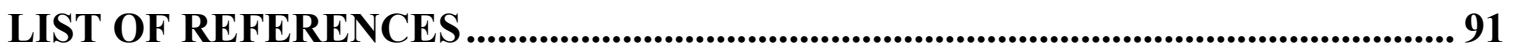

VITA 


\section{LIST OF FIGURES}

Figure 1.1

B cell development. ................................................................................... 3

Figure 1.2 Structure of immunoglobulin. ............................................................ 7

Figure 1.3 Structure of IgM pentamer. ……………............................................ 8

Figure 1.4 Glycosylation of the immunoglobulin Hc in the ER.............................. 11

Figure 1.5 Modification of the N-linked glycans on the immunoglobulin

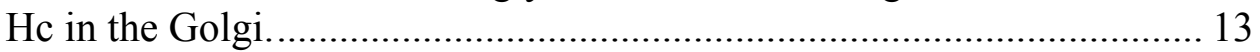

Figure 1.6 Receptor editing at the Lc locus........................................................... 19

Figure 2.1 Construction of bivalent variable fragments. ........................................... 34

Figure 2.2 $\mathrm{H}$ chain Tg BALB/c mice show drastic differences in their $\mathrm{L}$ chain

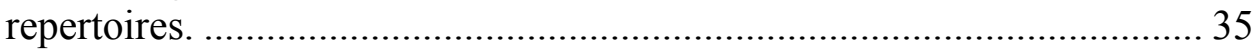

Figure 2.3 Binding of bivalent scFv to dsDNA and PS in ELISA. .......................... 37

Figure 2.4 Analysis of scFv binding to Jurkat cells................................................. 39

Figure 2.5 Distribution of IgM in B cell hybridomas and primary B cells................. 41

Figure 2.6 Surface $\kappa$ staining intensity in $\operatorname{IgM}^{\mathrm{a}}$ versus $\operatorname{IgM}^{\mathrm{b}}$ gated $\mathrm{B}$ cell populations from VH56R Tg C57BL/6 mice.............................................. 44

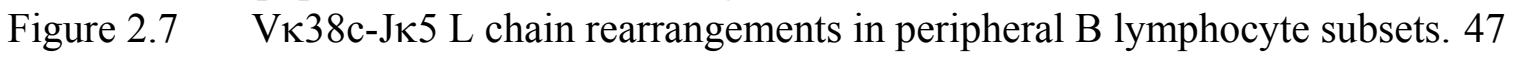

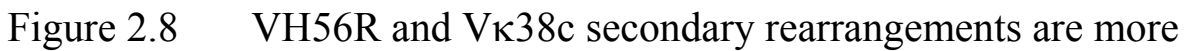
abundant in surface IgM ${ }^{\mathrm{a}}$-bright $\mathrm{B}$ cells. ................................................. 50

Figure 3.1 Distribution of IgM in B cell hybridomas and lymphomas.......................5 57

Figure 3.2 IgM accumulates in the Golgi in hybridomas. ........................................ 58

Figure 3.3 Golgi fragments after nocodazole treatment. ........................................... 62

Figure 3.4 Analysis of hybridomas and spherons by microscopy.............................. 63

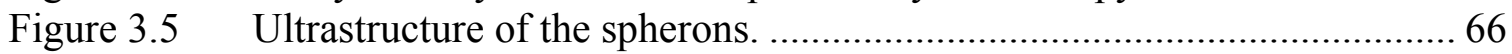

Figure 3.6 Rate of secretion, size and composition of the spherons............................ 67

Figure 3.7 Resolution and identification of intracellular IgM, secretory

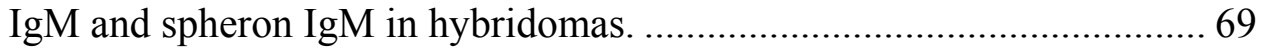

Figure 3.8 2-D Analysis of intracellular and secreted IgM. .................................... 72

Figure 3.9 Pulse-chase analysis of IgM synthesis. ................................................ 75

Figure 3.10 IgM fractions after glycosidase treatment................................................ 78 


\section{LIST OF ABBREVIATIONS}

\begin{tabular}{|c|c|}
\hline$\beta$-COP & $\beta$ Coat protein \\
\hline$\beta$-ME & $\beta$ mercaptoethanol \\
\hline $\mathrm{Ab}$ & Antibody \\
\hline $\mathrm{AF}$ & Alexa Fluor \\
\hline AGA & Anti-Golgi complex antibody \\
\hline AID & Activation induced deaminase \\
\hline ANA & Antinuclear antibody \\
\hline AP & Alkaline phosphatase \\
\hline APC & Allophycocyanin \\
\hline Apc & Antigen presenting cell \\
\hline ATF & Activating transcription factor \\
\hline $\mathrm{B} / \mathrm{W}$ & New Zealand black/New Zealand White mice \\
\hline $\mathrm{BCR}$ & B cell receptor \\
\hline BiP & Binding protein \\
\hline Blnk & $\mathrm{B}$ cell linker protein \\
\hline BM & Bone marrow \\
\hline BSAc & Acetylated bovine serum albumin \\
\hline $\mathrm{C}$ & Constant region \\
\hline $\mathrm{CD}$ & Cluster of differentiation \\
\hline cDNA & Cyclic DNA \\
\hline CDR & Complementarity determining region \\
\hline CGN & Cis-Golgi network \\
\hline $\mathrm{C}_{\mathrm{H}}$ & Heavy chain constant region \\
\hline CLL & Chronic lymphocytic leukemia \\
\hline CSR & Class Switch recombination \\
\hline $\mathrm{D}$ & Diversity region \\
\hline $\mathrm{D}_{\mathrm{H}}$ & Heavy chain diversity region \\
\hline DMSO & Dimethyl sulfoxide \\
\hline DSP & Dithiobis succinimidyl propionate \\
\hline DTT & Dithio threitol \\
\hline EDEM & ER degradation enhancing alpha mannosidase like protein \\
\hline EDTA & Ethelene diamine tetra acetic acid \\
\hline ELISA & Enzyme linked immunosorbent assay \\
\hline Endo $\mathrm{H}$ & Endoglycosidase $\mathrm{H}$ \\
\hline ER & Endoplasmic reticulum \\
\hline ERAD & ER associated degradation \\
\hline ERdj & ER DNAj like proteins \\
\hline ERGIC & ER Golgi intermediate compartment \\
\hline ERp & ER resident protein \\
\hline Fc $\gamma$ RIIb & Crystallizable fragment gamma receptor IIb \\
\hline FITC & Fluorescein isothiocyanate \\
\hline FO & Follicle \\
\hline GAGs & Glycosaminoglycans \\
\hline
\end{tabular}




\begin{tabular}{|c|c|}
\hline GlcNAc & $\mathrm{N}$-acetyl glucosamine \\
\hline GRP & Glucose regulated protein \\
\hline $\mathrm{H}$ & Heavy \\
\hline HBSS & Hanks basal salt solution \\
\hline $\mathrm{Hc}$ & Heavy chain \\
\hline HEL & Hen egg lysozyme \\
\hline HEPES & N-2-Hydroxyethylpiperazine-N'-2-ethanesulfonic acid \\
\hline HRP & Horse radish peroxidase \\
\hline Ig & Immunoglobulin \\
\hline IRE & Inositol requiring \\
\hline ITAM & Immunoreceptor tyrosine-based activation motif \\
\hline $\mathrm{J}$ & Joining \\
\hline $\mathrm{J}_{\mathrm{H}}$ & Heavy chain joining region \\
\hline $\mathrm{L}$ & Light \\
\hline Lc & Light chain \\
\hline LMP2A & Latent membrane protein $2 \mathrm{~A}$ \\
\hline LPS & Lipopolysaccharide \\
\hline MBP & Myelin basic protein \\
\hline MHC II & Major histocompatibility complex II \\
\hline MTOC & Microtubule organizing center \\
\hline $\mathrm{MZ}$ & Marginal zone \\
\hline OS9 & Amplified in osteosarcoma 9 \\
\hline PBS & Phosphate buffered saline \\
\hline PCR & Polymerase chain reaction \\
\hline $\mathrm{PE}$ & Phycoerythrin \\
\hline PERK & PKR like ER kinase \\
\hline PMSF & Phenylmethylsulphonyl fluoride \\
\hline PNGase F & Peptide: N-Glycosidase F \\
\hline PS & Phosphatidylserine \\
\hline $\mathrm{PtC}$ & Phosphatidyl choline \\
\hline RAG & Recombination activation gene \\
\hline RPMI & Roswell Park Memorial Institute medium \\
\hline RT-PCR & Reverse transcriptase Polymerase chain reaction \\
\hline $\mathrm{scFv}$ & Single chain variable fragment \\
\hline SDS-PAGE & Sodium dodecyl sulfate-polyacrylamide gel electrophoresis \\
\hline SHM & Somatic hypermutation \\
\hline SHP & Src homology region-2 domain phosphatase \\
\hline SLC & Surrogate light chain \\
\hline SLE & Systemic lupus erythematosus \\
\hline$S r c$ & Sarcoma \\
\hline Syk & Spleen tyrosine kinase \\
\hline TGF $\beta$ & Transforming growth factor $\beta$ \\
\hline $\mathrm{T}_{\mathrm{H}}$ cells & $\mathrm{T}$ helper cells \\
\hline UDP & Uridine diphosphate \\
\hline UGGT & UDP glucose glycoprotein glucosyltransferase \\
\hline UPR & Unfolded protein response \\
\hline
\end{tabular}


V

$\mathrm{V}_{\mathrm{H}}$

$\mathrm{V}_{\mathrm{L}}$

Variable region

Heavy chain variable region

Light chain variable region 


\section{CHAPTER 1: INTRODUCTION}

The autoimmune response is similar to the body's humoral immune response except that in autoimmunity, instead of tackling foreign antigens, the immune system targets the body's tissues themself. Thus the major components, B cells, T cells, macrophages and the dendritic cells, participate in both the normal and the abnormal immune responses. B cell receptors (BCR) are perhaps the most important elements that influence the fate of B cells from the early developmental stages in the bone marrow (BM) to the mature stages in the periphery. It is the BCR that are secreted as antibodies during the humoral immune response and during autoimmune disease as pathogenic autoantibodies. Therefore understanding B cell regulation and BCR is extremely important to illuminate the origins of autoimmunity and its pathogenesis once it is established. Mechanisms like clonal deletion, anergy and receptor editing prevent autoreactive B cells from reaching the periphery. In spite of this, some autoreactive B cells emerge out of the BM into the peripheral organs. One possible way for this to happen is by retention of the harmful receptor intracellularly by some mechanism while a harmless receptor in the same cell aids the B cell to move to the periphery. Nevertheless once an autoreactive $\mathrm{B}$ cell is out of the BM, it migrates to the lymph nodes and to the spleen. In autoimmune disease, these B cells get activated and become plasma cells to produce pathogenic autoantibodies.

The studies described in this dissertation have addressed the regulation of autoreactive B cells in mice. We started by constructing BCR analogs and used them to assess their affinity and specificity to auto antigens. The aim was to correlate this knowledge with possible fates of developing B cells that carry these receptors. During the course of the study we came across a very interesting receptor which in spite of being autoreactive was present in the periphery. Naturally, we were attracted towards the B cells that made these receptors. We have analyzed and tracked these B cells to the splenic marginal zone (MZ) and propose reasons for their presence in the periphery. B cells bearing this receptor showed another interesting property. They resembled 'Mott cells' with intracellular antibody accumulation. We extended our studies with hybridomas that synthesize these receptors as IgM antibodies. In these hybridomas, we discovered a spectacular new way of antibody secretion which might offer the explanation for the pathogenesis of autoantibodies in many autoimmune diseases. The general introduction in this dissertation reflects this progression of events, starting with role of B cells in humoral immune response. This is followed by the type of antibodies B cells secrete when they get activated in the periphery. The importance of BCRs for B cell survival and development is dealt with next. Following this, where autoreactive B cells might be present in the periphery is discussed. The subsequent sections deal with the mechanisms that prevent autoreactive B cells from migrating to the periphery. Finally the characteristics of systemic autoimmune disorders are discussed. 


\subsection{Life of a B cell}

B-lymphocytes are cells that play a crucial role in humoral immunity. B cells are produced throughout the lifespan of an individual in the bone marrow (1) (Figure 1.1). $2 \mathrm{X} 10^{7}$ cells are produced every day in mice and the cells circulate in the blood and lymph(2). B cells arise from common lymphoid progenitors and the earliest B cell precursors require contact with the stromal cells in the BM $(3,4)$, in addition to specific cytokines, particularly IL-7 $(5,6)$. B cells at later stages of development do not require cell contact, but need cytokines for further differentiation. Cell surface proteins such as the $200 \mathrm{kDa}$ protein, B220, are useful cell surface 'markers' and aid in identification of B cells (7). Each B cell also has a unique cell surface receptor called the BCR. The BCR along with co-receptors named $\operatorname{Ig} \alpha$ and $\operatorname{Ig} \beta$ form a complex $(8,9)$ which recognizes foreign antigens. Binding to antigen is the key initiating event that leads to antigenspecific B-cell differentiation and the development of humoral immunity.

B cells that complete development in the BM exit as immature B cells that migrate to the peripheral lymphoid organs - the spleen and lymph nodes. The majority of these immature B cells have a short life in the spleen (4 to 6 days), while some go on to the longer-lived recirculating B-cell compartment ( $\sim 60$ days). The mature recirculating B cells make up the naïve B cells found in periphery. These naïve B cells are referred to as conventional B cells or B-2 B cells. B-2 B cells, with help from another cell type called $\mathrm{T}_{\mathrm{H}}$ cells, generate responses to most foreign protein antigens. Two other distinct subsets, the B-1 B cells (10) and the MZ B cells (11) make up the long-lived B cells in the spleen.

A class of cells called dendritic cells is required for the induction of B- and T-cell responses in vitro and in vivo. Dendritic cells, upon interaction with antigen, become activated and migrate to the lymph nodes. Dendritic cells are a type of antigen presenting cells. Dendritic cells take up antigen, process the antigen and load the antigenic peptide fragments onto a molecule called the major histocompatibility complex II (MHC II) (12, 13). Naïve antigen-specific $T_{H}$ cells and dendritic cells form an immune synapse. Costimulatory molecules, adhesion molecules and molecules involved in antigen stimulation on both cell types form the synapse. Activated $\mathrm{T}_{\mathrm{H}}$ cells then expand clonally and migrate to the secondary lymphoid organs(14). Antigen-specific naïve B cells are also recruited into this pathway. To receive cognate T-cell help, antigen-specific B cells must have contacted their specific antigen, internalized, processed, and presented antigenic peptides on their cell surface in association with MHC class II (15). Once the B cells are activated, they too relocate to the secondary lymphoid organs to increase the likelihood of acquiring cognate T-cell help. Thus, at around 5 to 7 days after initial antigen contact, both the antigen-activated-effector $\mathrm{T}_{\mathrm{H}}$ cells and antigen-primed $\mathrm{B}$ cells are translocated to the same microenvironment.

When the antigen activated B cells in the follicles of secondary lymphoid organs come in contact with the antigen specific $\mathrm{T}_{\mathrm{H}}$ cells, an immune synapse forms here and the $\mathrm{B}$ cells begin to divide rapidly and form secondary follicles. One group of B cells become short lived IgM secreting plasma cells while the other group initiates what is called the germinal center reaction (16). Plasma cells are terminally differentiated and secrete 


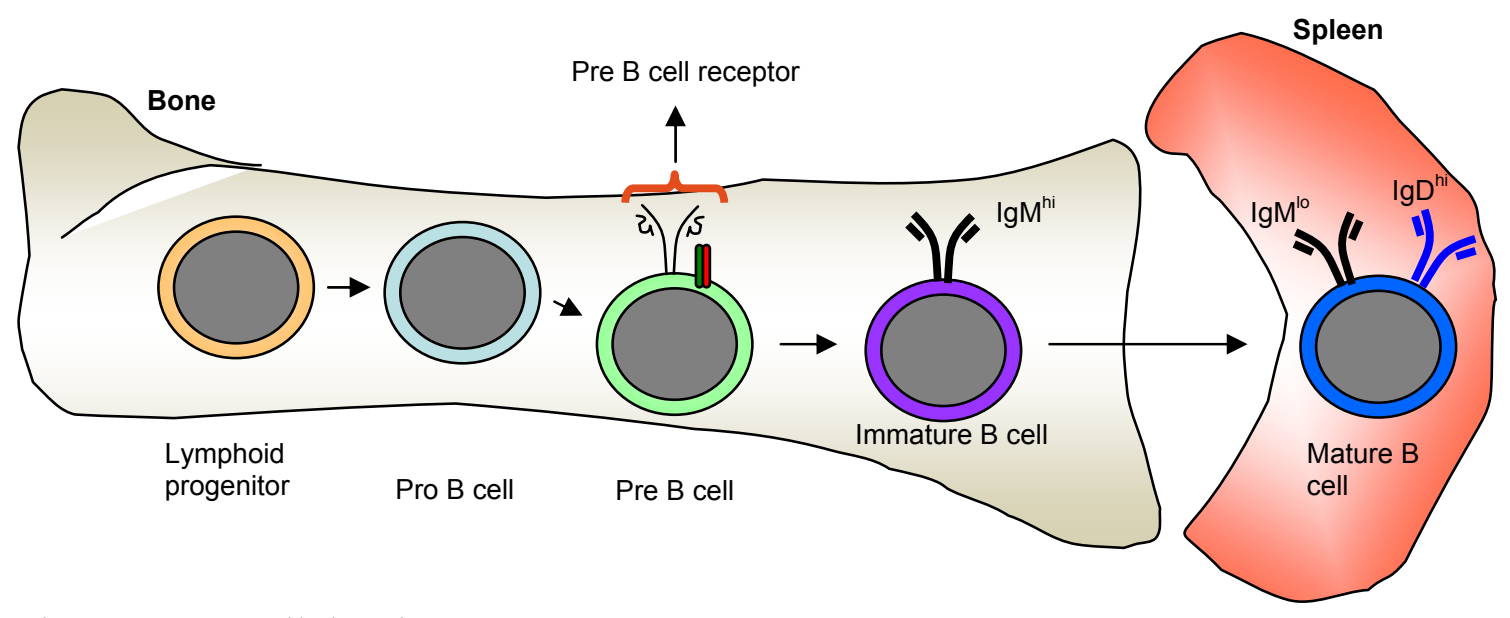

Figure 1.1 B cell development. 
antigen specific antibody in large amounts (17) while B cells in the germinal center reaction undergo immunoglobulin (Ig) gene diversification processes called class switch recombination (CSR) and somatic hypermutation (SHM) (18).

B-lymphocytes are cells that play a crucial role in humoral immunity. B cells are produced throughout the life span of an individual in the BM (1) (Figure 1.1). In the presence of CD40, a co-stimulatory molecule and its ligand (CD-40L) (19) there is a class switch in antibody secretion from IgM to IgG, IgE or IgA (20). A soluble T cell derived factor IL-4 also has a role in Ig CSR (21). CSR replaces an initially expressed constant region of an Ig heavy chain with an alternate heavy chain constant region. Therefore after class switching the antibody has the same antigen specificity but a different heavy chain isotype. In CSR specialized regions of DNA named switch regions that exist 5 ' to each constant region gene in the $\mathrm{B}$ cells are cut and rejoined after deleting the intervening region (22). This process is aided by a DNA editing cytidine deaminase named activation induced deaminase (AID) (23). Other proteins involved in DNA repair (24) and DNA joining (25) play important roles in Ig class switching.

B cells undergo affinity maturation and SHM to generate high affinity antibodies. SHM introduces non templated mutations in the variable region of rearranged Ig heavy and light chain genes. Point mutations occur predominantly during SHM and SHM is influenced by the primary sequence of DNA (26). SHM is initiated by the deamination of cytidines by AID. The resulting $\mathrm{U} \bullet \mathrm{G}$ mismatch if left un-repaired can result in transition mutations. However, error prone repair processes themselves can introduce mutations (27).

\subsection{B cell development}

Early B cell developmental stages are BCR independent and precede antibody gene recombination (28). The earliest committed B cell precursors are pre-pro B cells (Figure 1.1). These cells have not rearranged their Ig gene loci. Pre-pro B cells differentiate to pro-B cells (29). Ig gene recombination is initiated at the pro-B cell stage with the $\mathrm{D}_{\mathrm{H}^{-}} \mathrm{J}_{\mathrm{H}}$ rearrangement. Recombination is brought about by Recombination activation gene 1 (RAG1) and RAG 2 genes (30). Due to a property of random nucleotide addition or loss, the $\mathrm{D}_{\mathrm{H}}$ segments can combine with $\mathrm{J}_{\mathrm{H}}$ in any of the three available reading frames. Usually $\mathrm{D}_{\mathrm{H}^{-}} \mathrm{J}_{\mathrm{H}}$ rearrangements in reading frame 2 are truncated, nonfunctional and incapable of supporting further $\mathrm{B}$ cell differentiation, in the absence of continuing recombinations (31). When $\mathrm{D}_{\mathrm{H}^{-}} \mathrm{J}_{\mathrm{H}}$ recombination is complete the $\mathrm{V}_{\mathrm{H}}$ genes become accessible to be combined with D-J, to give rise to the VDJ part of the heavy chain $(\mathrm{Hc})(32,33)$. The resulting RNA transcript has the VDJ along with $\mathrm{C}$ region and a splicing step gives rise to VDJC to give the complete Hc (34).

IgM Hc expressed on the cell membrane marks the transition from the pro-B to the pre-B cell stage. Two components named $\mathrm{V}$ pre $\mathrm{B}$ and $\lambda 5$ come together to form the surrogate light chain $(\psi L c)$ to associate with the Hc (35). The pre-BCR has the membrane $\operatorname{Ig} \mu, \psi \operatorname{Lc}, \operatorname{Ig} \alpha$ and $\operatorname{Ig} \beta$ (36). The pre-BCR exists as a complex with calnexin. 
$\operatorname{Ig} \alpha$ and $\operatorname{Ig} \beta$ are signaling components that associate with the $\mathrm{BCR}$ as an immature complex $(37,38)$ and are required for the surface expression of membrane $\operatorname{IgM}(39,40)$. Ig $\alpha$ and $\operatorname{Ig} \beta$ in turn activate B cell signaling pathways through ITAMs by recruiting $\mathrm{Src}$ and $S y k$ family kinases $(41,42)$. The pre-BCR triggers B cell differentiation and clonal expansion. Deleting pre-BCR components blocks B cell development at the pro-B cell stage (43). However inactivation by targeted gene disruption of $\lambda 5$, one of the component $\psi \mathrm{Lc}$ associated with the $\mu \mathrm{Hc}$, produces a leaky phenotype that allows some B cell development (44). This probably can be explained by the substitution of surrogate light chains by early Lc rearrangements (45). Receptor aggregation probably is required in addition to the non-redundant signaling components $S y k$, Blnk and PI3K for full B cell development (46-48). The exact mechanism of pre-BCR signaling is not clear. The current model is that the pre-BCR signaling is similar to "tonic" signaling in mature B cells. That is, just the assembly of the complex may be sufficient for the cell to pass the pre-B cell developmental checkpoint (49).

Allelic exclusion of immunoglobulin genes ensures the expression of a single antibody molecule in B cells. In allelic exclusion, after an initial rearrangement event occurs, simultaneous recombination of the second allele is prevented by different feedback mechanisms. First, this feedback mechanism brings about the down regulation of RAG1, RAG2 and TdT (50). Pre-B cell signaling brings about reorganization of the heavy chain locus resulting in the prevention of further rearrangements at the second DJH allele (51). After pro-B cells undergo rearrangement at the heavy chain locus, they enter into a proliferative stage that is aided by IL-7 signaling. During the transition to small pre-B cells, this pathway is down regulated, leading to histone deacetylation at the IgH locus (52). This then leads to a decrease in accessibility of Hc genes, which serves to prevent any further recombination even though these same cells are actively undergoing IgL rearrangement.

Hc genes are arranged linearly on the chromosome and certain proximal $\mathrm{VH}$ genes are over-represented in B cells at the fetal stages. However, in adults there is selection against proximal VH genes and those with long complementarity determining regions (CDR3s) because Hc with long CDR3s have a higher probability of being autoreactive (53). Lc genes start undergoing recombination at the pre-B cell stage and the newly expressed Lc replace the $\psi \mathrm{Lc}$ on the pre-BCR (54). There are two Ig Lc isotypes encoded by separate gene families, $\kappa$ and $\lambda$, but normally B cell progenitors functionally rearrange and express only one Lc gene to generate B cells which are either $\kappa$ or $\lambda$ (5557). In the mouse genome, the $\kappa$ Lc family is substantially more complex than the $\lambda$ gene family and therefore there is a bias towards the expression of the $\kappa$ isotype such that approximately $95 \%$ of the B cells will be $\kappa^{+}(58) . \kappa$ Lc are often rearranged in $\lambda$ producers but not vice versa which means that $\kappa$ genes are generally rearranged before $\lambda$. $\lambda$ rearrangements are increased when one or both $\kappa$ loci are inactivated. However $\lambda$ genes do rearrange in $\kappa^{+/+}$cells (59). 


\subsection{B cell receptors}

Antibodies neutralize foreign bacterial and viral antigens. Antibodies prevent pathogens from entering into cells, aid in the removal of pathogens and initiate the destruction of the pathogens via the complement pathways $(60,61)$. In mammals there are antibody classes or isotypes and they differ in their biological properties, location and capacity to deal with different antigens. Antibodies can be monomeric, dimeric or pentameric depending on the number of Ig units present. Depending on the type of $\mathrm{Hc} \mathrm{Ig}$ belong to five different classes, $\operatorname{IgM}, \operatorname{IgD}, \operatorname{IgG}, \operatorname{IgA}$ and $\mathrm{IgE}$ (Figure 1.2). The heavy chains are named by Greek letters and they differ in size and composition. $\alpha$ and $\gamma$ have about 450 amino acids, while $\mu$ and $\varepsilon$ have about 550 amino acids (62-64).

The BCR is an Ig embedded in the cell membrane. The Ig or the antibody is unique for each B cell and it is a hetero tetramer composed of two Hc and two Lc. The variable regions of each $\mathrm{Hc}+\mathrm{Lc}$ form an antigen binding site. The genes specifying the $\mathrm{H}$ and the L chains are found at the Hc locus and the Lc locus. The Hc is encoded by four types of gene segments. They are variable (V), diversity (D), joining (J) and constant (C) region genes (65). Multiple copies of the $\mathrm{V}, \mathrm{D}$, and $\mathrm{J}$ genes are arranged tandemly in the genome.

The Lc is encoded by the V, J and the C genes. Mouse $\kappa$ locus has 197 functional $\mathrm{V} \kappa$ and four functional $\mathrm{J} \kappa$ segments. There are two types of Lc, also named by Greek letters, the $\kappa$ and $\lambda$. Lc are made up of only two Ig domains which make up the constant and variable regions. As there are multiple gene segments that can be used to form the Hc and the Lc, different rearrangements can theoretically generate an antibody repertoire of $>10^{7}$ combinations.

Each Hc has a variable region and a constant region. The variable region is different for every BCR but it is the same for all the antibodies produced by a single B cell or its clones. A single domain makes up the variable region (66). The part of the antibody that binds to antigens is called Fab' (67) and the part which modulates immune cell function is called the Fc region (68). The Fc region binds to Fc receptors on other cells, and to complement.

\section{$1.4 \operatorname{IgM}$}

$\operatorname{IgM}$ is important for combating infectious diseases because of its high capacity for activating the complement pathway. IgM must be polymerized into pentamers of the basic heterotertamers (Figure 1.3) if it is to activate complement (69). IgM also mediates opsonization. Opsonization is the coating of the antigen by antibody that facilitates uptake of the antigen by phagocytosis. The polymers have more avidity for antigens than the monomers. The $\mathrm{CH} 3$ domain of IgM binds to the $\mathrm{C} 1 \mathrm{q}$ complement component. IgM's $\mathrm{CH} 1$ domain interacts with $\mathrm{C} 3 \mathrm{~b}$ complement component and helps in phagocytosis of opsonized antigens (70). 


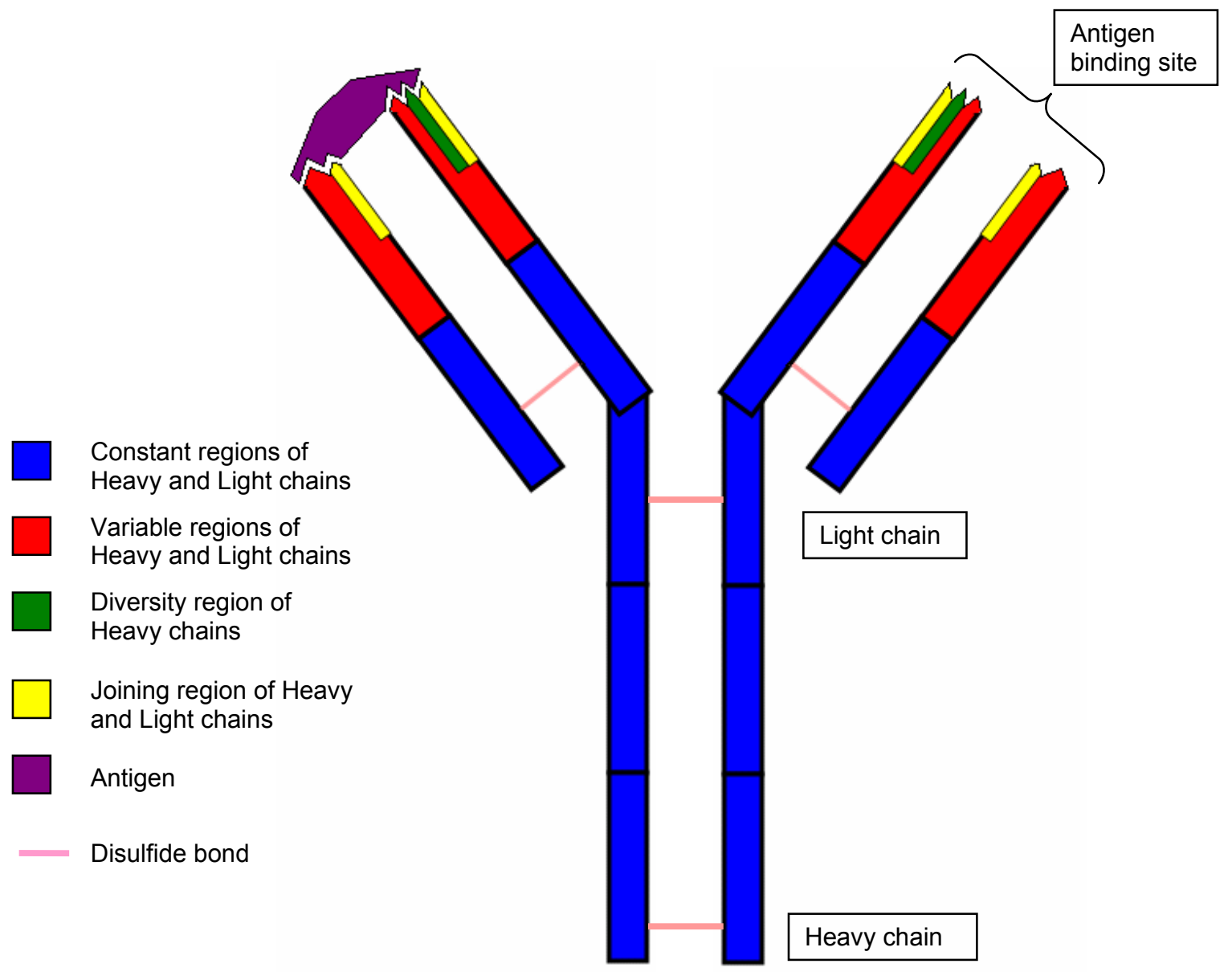

Figure 1.2 Structure of immunoglobulin. 


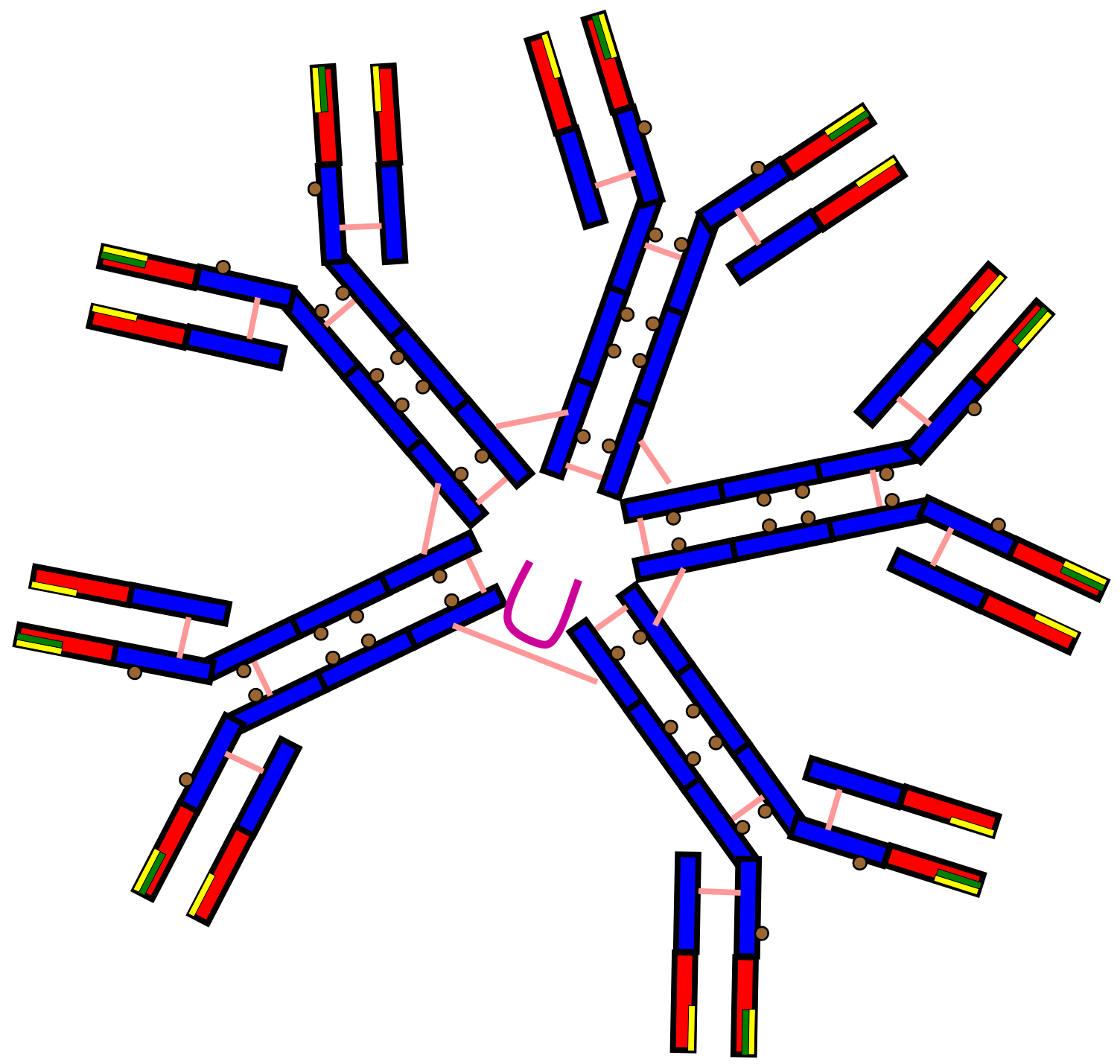

Constant regions of Heavy and Light chains

Variable regions of Heavy and Light chains

Diversity region of Heavy chains

Joining region of Heavy and Light chains

\section{Disulfide bond}

$\int J$ chain

Location of carbohydrate side chains

Figure 1.3 Structure of IgM pentamer. 
The $\mu$ Hc can be produced in two forms, one $\left(\mu_{\mathrm{m}}\right)$ which gets inserted into the B cell membrane (71) because of a hydrophobic C-terminal portion and the other $\left(\mu_{\mathrm{s}}\right)$ with a shorter hydrophilic $\mathrm{C}$ terminal that gets secreted by the plasma cells (72). The two forms are generated by distinct mRNAs that arise due to alternate splicing/processing of the primary $\mu$ gene transcript. The secreted $\mu_{\mathrm{s}}$ terminates with a tail piece that has 20 hydrophilic residues and includes a glycosylation site at asparagine-563 and a cysteine 575 residue that is used in the formation of a disulfide bond. This is replaced in $\mu_{\mathrm{m}}$ by 41 amino acids that include the hydrophobic transmembrane domain (73). In plasma cells monomers of $\operatorname{sIgM}\left(\mu_{2} \mathrm{~L}_{2}\right)$ are assembled into polymers $\left(\mu_{2} \mathrm{~L}_{2}\right)_{5}$ and only polymers are secreted (74).

\subsubsection{IgM assembly}

Human Burkitt lymphoma line Ramos was used as a model to study the assembly and intracellular transport of the BCR complex (75). The cells are mature IgM bearing cells. Ig are glycoproteins produced on the rough ER and transported into the ER lumen. The order of assembly of the components was determined by labeling the cells for 3 minutes and chasing for an hour. In this cell line, $\mu \mathrm{Hc}$ is made in excess compared to Lc and association of the Hc with the Lc occurs independently of interaction with the $\operatorname{Ig} \alpha / \operatorname{Ig} \beta . \mu \mathrm{Hc}$ associate with the Lc almost immediately after they are synthesized. $\mathrm{H} / \mathrm{L}$ dimers (also called hemimers), at $110 \mathrm{kD}$ are also observed on non-reducing gels. The $\mathrm{H}_{2} / \mathrm{L}_{2}$ tetramer (also Ig monomer) formation occurs within the first few minutes after biosynthesis and is complete after about $2 \mathrm{hrs}$. The tetramer becomes Endo H resistant only after about $4 \mathrm{hrs}$. Resistance to the enzyme Endoglycosidase $\mathrm{H}$ indicates that secreted protein has passed through the Golgi. This implies that assembly of the $\mathrm{H}_{2} / \mathrm{L}_{2}$ tetramer precedes exit out of the ER. $\mu \mathrm{Hc}$ associated with Ig $\alpha$ appears to be more efficiently transported out of the ER into the Golgi (75).

The Golgi apparatus is a series of flattened, hollow cisternae composed of membranes forming the secretory pathway. These cisternae, unlike the endoplasmic reticulum, do not fuse into one continuous reticular structure, but are present as a stack. The Golgi stack is a polarized structure with a cis-face exchanging proteins and lipids with the endoplasmic reticulum (ER), and a trans-face communicating with the plasma membrane and compartments of the endocytic pathway. Complex networks referred to as the cis-Golgi network (CGN) and the trans-Golgi network (TGN) respectively are found at each of these faces $(76,77)$. Secretory proteins pass through the Golgi cis to trans and their N-linked glycans become post-translationally modified in a sequential order before being sorted at the TGN for delivery to their final destination within the cell. Golgi is a highly dynamic organelle despite its highly organized structure (76). Compartment identification can be done by anti-ER antibodies or anti-Golgi antibodies. 


\subsubsection{Importance of $\mathrm{J}$ chain for assembly into polymers}

Antigen and the successive cytokine signals trigger resting B cells to secrete IgM pentamers. Synthesis of J chain is a critical step in IgM pentamer secretion (78). The J chain links two monomer subunits (Figure 1.3) through disulfide bonds between cysteines at positions 14 and 68 of the $J$ chain and the penultimate cysteines of opposing Hc to form the pentamers (79). The tail piece of the IgM has this penultimate cysteine and there is also an asparagine which is glycosylated. This glycosylation is also important for $J$ chain incorporation and pentamer formation in $\operatorname{IgM}$. The $\mu$ tail piece must have the $\mathrm{CH} 3, \mathrm{CH} 4$ domains for the $\mathrm{J}$ chain to be incorporated. In the absence of the J chain, an additional monomer closes this circle to form the hexamer (80). Similarly, elimination of carbohydrate in the $\mu \mathrm{Hc}$ leads to formation of higher order polymers (81). Since IgM assembly occurs through stepwise addition of Hc/Lc hemimers, it limits the accessibility of glycosyl transferases in the $\mu$ tail piece. Therefore incorporation of $\mathrm{J}$ chain into polymeric Igs is not only regulated by the amount of $\mathrm{J}$ chain but also features of the constant domains and the $\mu$ tail piece. The production of IgM hexamers has been shown to activate complement-dependent cytolysis 17 - to 20 -fold more efficiently than J-chaincontaining pentamers and thus may have an important role in immune responses (82). Presence of IgM hexamers in the body can be detrimental. An example to support this is the presence of significant amount of IgM hexamers in the sera of patients with cold agglutinin disease, an IgM mediated autoimmune condition (82). The normal B splenocyte is a resting cell of the B2 subset that synthesizes only the membrane form of $\mu$ chain $\left(\mu_{m}\right)(83)$ and requires antigen and lymphokine stimulation to undergo a shift to the secreted form $\left(\mu_{\mathrm{s}}\right)$. Lipopolysaccharide activated splenic MZ B cells show that the shift is initiated within $24-48 \mathrm{hr}$ after stimulation and proceeds to completion at $72 \mathrm{hr}$. J chain can be detected within 48-72 hr after LPS stimulation (84).

\subsubsection{Glycosylation of IgM H chains}

Glycosidases play a vital role in the development of hybrid and complex types of $\mathrm{N}$-linked oligosaccharides (85). N-linked oligosaccharides are initially assembled in the endoplasmic reticulum by the stepwise addition of various sugars to a lipid carrier, named dolichol-P (Figure 1.4). This oligosaccharide part gets transferred to specific asparagine residues on the polypeptide chain as the protein is being synthesized on the ribosomes. After the oligosaccharide has been transferred to protein, it undergoes processing. As a result some sugars are removed and while others are added. The initial reactions occur in the endoplasmic reticulum (86). This involves the removal of all three glucose residues by two membrane-bound $\alpha$-glucosidases. Glucosidase I removes the outermost $\alpha 1,2$ linked glucose. The second $\alpha$-glucosidase, called glucosidase II, removes the other two $\alpha$ 1,3-linked glucose residues (87). ER $\alpha$-mannosidase acts on high-mannose oligosacchanides. The high-mannose structure that results from these reactions may remain as a high mannose glycoprotein and avoid further trimming or it may be processed further as the glycoprotein travels through the various Golgi stacks (Figure 1.5) (88). In proteins where the oligosaccharide remains as a high-mannose structure and escapes further processing, it is likely that the carbohydrate chain is buried within the 
Figure 1.4 Glycosylation of the immunoglobulin Hc in the ER.

Biosynthesis of the N-linked core oligosaccharide. Synthesis starts on the cytosolic surface of the ER membrane by the addition of sugars, one by one, to dolicholphosphate. When two $\mathrm{N}$-acetylglucosamines and five mannoses have been added, the oligosaccharide is flipped to the lumenal side of the membrane, and seven further sugars are added from lipid precursors. After the last of the three glucoses have been added, the oligosaccharyltransferase enzyme complex catalyzes the transfer of the core oligosaccharide to the asparagine residues of nascent, growing polypeptide chains. The three glucoses are trimmed away by glucosidase I and II, and terminal mannoses by one or more different ER mannosidases. The ER also contains a glucosyltransferase that can reglucosylate glucose-free chains and thus establish, with glucosidase II, a deglucosylation- reglucosylation cycle. (Adapted with permission from Helenius A and Aebi M. (2001) Intracellular functions of N-linked glycans. Science Mar 23 (291) 5512:2364-9).

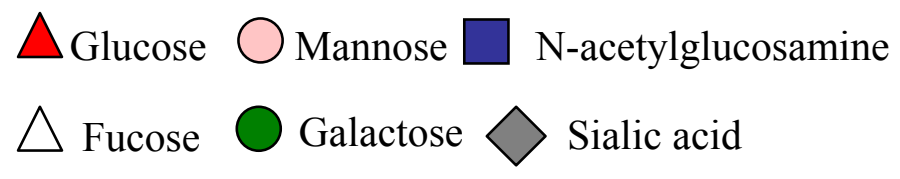

\footnotetext{
$\longrightarrow$ Glucosidase I, $\longrightarrow$ Glucosidase II, $\longrightarrow$ Endomannosidase $\longrightarrow$ ER $\alpha$-Mannosidase, $\longrightarrow$ Endoglycosidase $\mathrm{H}$
} 


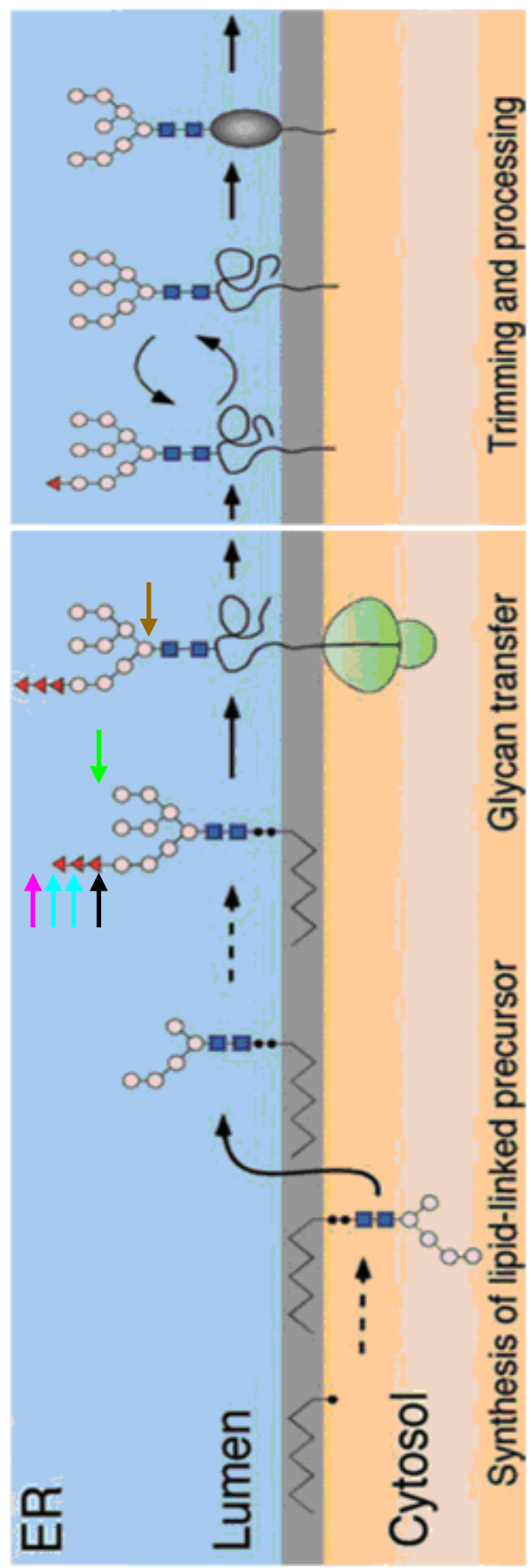


Figure 1.5 Modification of the N-linked glycans on the immunoglobulin Hc in the Golgi.

When the glycoprotein has folded (gray oval) and reached the Golgi complex, further mannose trimming occurs. The addition of a GlcNAc residue is followed by trimming of two additional mannoses. During subsequent terminal glycosylation there is addition of new terminal sugars including GlcNAc, galactose, sialic acid, and fucose. Of the original core glycan, just five sugars remain. Only one of many possible terminal glycosylation pathways is shown; the number of branches generated is variable, as are the number and identity of sugars added. Whereas the glycoforms in the ER are homogeneous, the Golgigenerated forms are highly diverse and differ widely between species. (Adapted with permission from Helenius A and Aebi M. (2001) Intracellular functions of N-linked glycans. Science Mar 23 (291) 5512:2364-9).

\section{$\triangle$ Glucose $\bigcirc$ Mannose $\square$ N-acetylglucosamine \\ $\triangle$ Fucose $\bigcirc$ Galactose $\diamond$ Sialic acid}




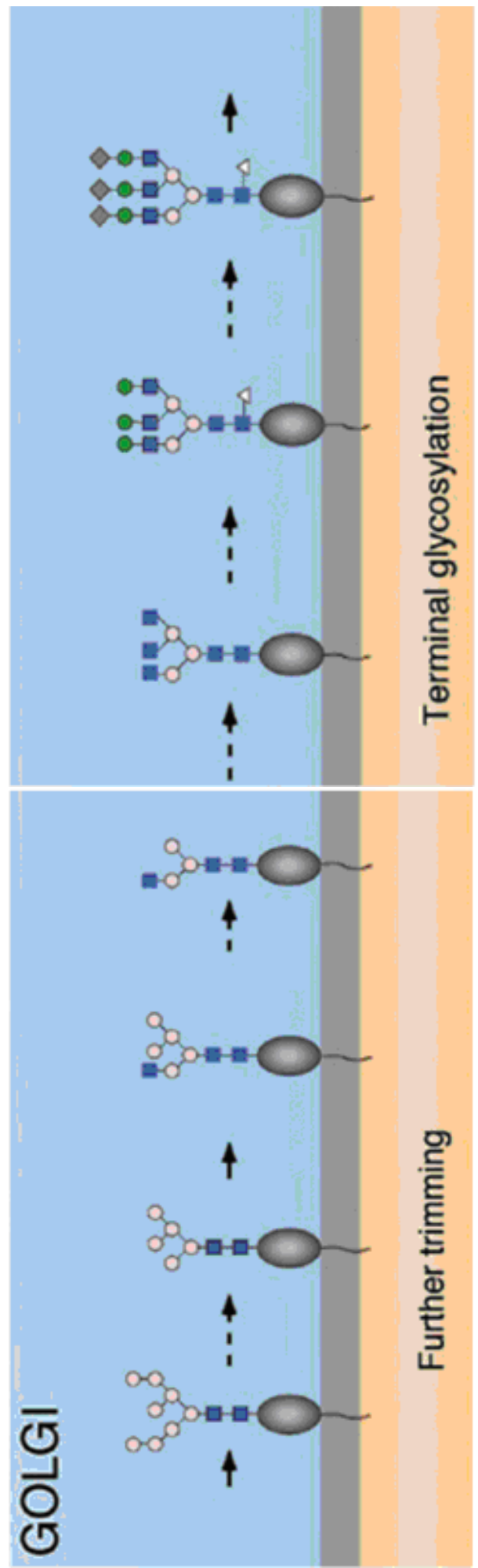


protein folds and/or is somehow inaccessible to the processing mannosidases. The glycoproteins present in the ER bear immature N-linked glycans that are sensitive to Endo $\mathrm{H}$ digestion. Resistance to Endoglycosidase $\mathrm{H}$ is acquired after transport to the medial-Golgi.

\subsection{Quality control for protein folding}

Proteins are covalently modified in the ER by cleavage of the signal peptide, Nglycosylation, formation of disulfide bonds and acquisition of their proper tertiary and, in some cases, also quaternary structures (89). N-glycosylation allows for quality control of glycoproteins in the ER. After synthesis the enzyme UDP glucose glycoprotein glucosyltransferase (UGGT) adds a glucose residue (90) to N-glycans positioned near misfolded regions thus acting as folding sensor that produces monoglucosylated proteins that can bind to ER chaperones calnexin and calreticulin (91). Calnexin and calreticulin retain misfolded proteins in the ER, prevent their aggregation and promote folding via interactions with ERp57 (92). The enzyme Glucosidase II removes this glucose from the protein for it to be inspected by UGGT again. Glucose addition can be prevented by removing the terminal mannose residues. Mannose trimming (by ER Mannosidase I and II, EDEMs, and Golgi mannosidases) thus acts as a timer that allows proteins either to be secreted or degraded (93). Further mannose trimming in the ER may allow retrotranslocation of the proteins out of the ER for degradation (94). Binding protein (BiP), a chaperone belonging to the Hsp70 family is another folding pathway that aids in protein folding (95). BiP has ATPase activity and substrate binding depends on ATP binding (96). ATPase cycle in BiP is regulated by ER localized DnaJ homologues (ERdj 1 to 5). The ERdj family of proteins stabilizes the interaction of BiP with unfolded substrates (97). Efficient folding may also require disulfide transfer from protein disulfide isomerases (PDI) to nascent cargo proteins. Disulfide bond formation is very important for folding and quality control of secretory proteins as this increases the stability of the protein's native conformation (98). Another protein with lectin activity, ERGIC-53 that cycles between the ER and Golgi, transports high mannose cargoes (example; IgM polymers) allowing their secretion (99). ERp44, another scaffold protein, accumulates in the ERGIC and cis-Golgi and mediates the retention of proteins (eg; IgM assembly intermediates) that have passed the earlier BiP dependent folding stages (100).

If protein folding in the ER is disrupted, as during a chemical insult or nutrient deprivation, it activates a stress signaling pathway known as the unfolded protein response (UPR). UPR induction results in both an initial decrease in general protein synthesis, to reduce the influx of newly synthesized proteins into the ER, and increased transcription of ER resident chaperones, folding enzymes, and components of the proteasomal pathway to prevent the aggregation of the accumulating misfolded proteins (101). These misfolded proteins are recognized by ER quality control mechanisms and retained in the ER, preventing them from proceeding further through the protein maturation process (102). In mammalian cells, three ER transmembrane components, IRE1, PERK, and ATF6, monitor protein folding in ER and initiate UPR activation (103). Terminally misfolded proteins escape the ER and are sent to ER associated degradation 
(ERAD). The misfolded proteins are recognized by EDEM 1, 2 and 3 (mannosidase like proteins) (104), OS-9 and XTP3-B which then bind to other molecules involved in ERAD. This results in retrotranslocation of the proteins marked for ER followed by their ubiquitination in the cytosol. ERAD is sometimes unable to clear mutant proteins possibly because the rate of synthesis exceeds the degradation capacity of a cell (105).

\subsection{Intracellular immunoglobulin accumulation}

Mutant Ig accumulation in ER is referred to as Russell bodies. Cells that harbor Russell bodies are called Mott cells (106). The Mott cell phenotype has been recreated in lymphoid and non-lymphoid cells by overexpressing $\mu \mathrm{Hc}$ that lack the first constant domain. Presence or absence of Lc however appears to determine the intracellular location of Russell bodies in cells. In the presence of Lc, the mutant $\mu \mathrm{Hc}$ aggregate in the rough ER (rER) and in the absence of Lc the mutant $\mu \mathrm{Hc}$ aggregate in vesicles associated with ERGIC-53. Spontaneous autoimmune viable motheaten $\left(m e^{v} / m e^{v}\right)$ mice have Mott cells that contain Ig accumulation in the rER. 31\% of the hybridomas constructed from cervical lymph node cells from motheaten mice had Russell bodies. Intracellular Ig remained insoluble in $0.5 \% \mathrm{NP}-40$. This intracellular Ig was able to be solublized by homogenizing the material in 1\% SDS. The intracellular Ig was Endo $\mathrm{H}$ sensitive consistent with localization of Ig to the rER.

\subsection{Mechanisms of B cell tolerance}

BCRs are generated by random rearrangements of the Ig genes. Since the receptors are generated randomly, it is impossible to predetermine which receptors will lead to anti-self activity. The B cells acquire tolerance to self components during their development by sampling the antigens in their environment. 'Tolerance' is defined as a physiologic state in which the immune system does not react against self antigens. Failure of tolerance mechanisms during B cell development leads to autoimmunity characterized by production of autoantibodies that react against self components. About $75 \%$ of newly made immature $\mathrm{B}$ cells in the $\mathrm{BM}$ are autoreactive and face a rigorous negative selection process (107). The evidence for autoreactive $\mathrm{B}$ cells undergoing negative regulation came from pioneering experiments by Nemazee and Burki when they constructed a transgenic mouse expressing a cell surface IgM (sIgM) BCR reactive against MHC class I molecules $\mathrm{H} 2 \mathrm{~K}^{\mathrm{k}}$ and $\mathrm{H} 2 \mathrm{~K}^{\mathrm{b}}$. In the absence of the autoantigen, B cells expressing class I MHC BCR were observed in the periphery, but in the presence of autoantigen B cells expressing class I MHC BCR disappeared. The site of negative selection was inferred to be the BM because the transgenic receptor bearing $\mathrm{B}$ cells were not detected in the spleen $(108,109)$. Hence clonal deletion was proposed as a mechanism of negative selection of autoreactive B cells.

Goodnow developed transgenic mice expressing IgM BCRs against hen egg lysozyme (HEL). Anti-HEL transgenic mice models confirmed Nemazee and Burki's results that B cells are clonally deleted, but Goodnow demonstrated that the B cells are 
deleted only when the antigens are membrane bound (110). Goodnow's experiments uncovered another mechanism called functional anergy when B cells expressing antiHEL receptors mature in mice that had soluble self antigens instead of a membrane bound form (111). The anti-HEL B cells were not deleted but were temporarily inactivated and were in a state of maturational arrest. Maintaining tolerance may be influenced by genetic background (112) and these experiments confirm that exposure to antigen is generally deleterious to developing B cells.

In both the systems described above, the self reactive transgenic B cells expressed receptors against 'non-disease-associated antigens'(113). Erikson et al, created the first anti-DNA transgenic mice on a BALB/c (non autoimmune) background using the $3 \mathrm{H} 9$ Hc. The $3 \mathrm{H} 9$ antibody has features of typical lupus autoantibodies in that it binds to nuclei, chromatin, DNA and phospholipids. The $3 \mathrm{H} 9 \mathrm{H}$ chain was repeatedly encountered in spontaneously arising anti-nuclear antibodies (114). Arginine mutations occur frequently in the $\mathrm{H}$ chain genes of anti-DNA antibodies in the MRL/lpr mice and additional arginines in the $3 \mathrm{H} 9 \mathrm{H}$ chain contributed to an increase in the $\mathrm{H}$ chain affinity to DNA and phospholipids (115). The productively rearranged 3H9 VDJ region from a $\mathrm{MRL} / \mathrm{lpr}$ (autoimmune prone mouse strain) anti-DNA hybridoma was joined to the $\mathrm{C} \mu$ fragment from a $\mathrm{BALB} / \mathrm{c}$ mouse. The entire construct was microinjected into pronuclei of fertilized embryos to obtain founder animals. $3 \mathrm{H} 9 \mathrm{H}$ chain only transgenic mouse is the progeny of one of the founder animals (114). These mice produced B cells with receptors specific for DNA, a main target antigen in systemic lupus erythematosus (SLE). These are Hc only transgenic mice and therefore B cells would express the anti-DNA Hc but any endogenous Lc can associate with the Hc. However the L chains have to be such that, they in association with the anti-DNA Hc must be able to prevent autoreactivity. The 3H9

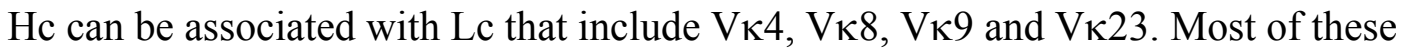
antibodies can bind to both ssDNA and dsDNA while $3 \mathrm{H} 9 / \mathrm{V} \kappa 8$ binds to ssDNA only. Thus due to the dominant anti-DNA nature of the $3 \mathrm{H} 9 \mathrm{Hc}$ the regulation of different kinds of anti-DNA antibodies was studied here. In addition to the Hc only transgenic mice, $3 \mathrm{H} 9 \mathrm{Hc} / \mathrm{V} \kappa 8 \mathrm{Lc} \mathrm{H} / \mathrm{L}$ transgenic mice were also created to analyze how anti-DNA B cells are regulated in normal mice. Site directed $3 \mathrm{H} 9 \mathrm{H}$ chain $\mathrm{Tg}$ mice have also been created (116). High numbers of anti-DNA B cells were found in the $3 \mathrm{H} 9 / \mathrm{V} \kappa 8$ mice but the anti-DNA serum titres were not higher compared to control mice. This suggested that anti-DNA B cells do not differentiate into Plasma cells and have become anergic. In contrast to B cells in the HEL and MHC models the anergic B cells have a high density of surface IgM. In the $3 \mathrm{H} 9 \mathrm{Hc}$ only transgenic mice there were no B cells that bound dsDNA. This suggests that anti- dsDNA B cells are deleted. About $60 \%$ of the B cells bound ssDNA but anti-DNA serum antibodies were not increased. These results showed that anti-DNA antibodies are regulated in non-autoimmune animals and that autoimmunity would result in the event of a dysregulation in autoantibody production (113).

\subsection{Receptor editing}

One important B cell tolerance mechanism by which the autoreactive B cells are prevented from making autoantibodies was elucidated by transgenic mice studies. 
Autoreactive B cells expressing anti-DNA receptors in transgenic mice are either anergized or deleted. However, B cell numbers appeared to be normal. Analysis of B cells showed that the autoreactive BCR has been replaced with a non-autoreactive receptor (117). This is termed "receptor editing".

Receptor editing alters antigen receptors by ongoing rearrangements in the BCR encoding elements (Figure 1.6). Receptor editing is a mechanism by which immature bone BM B cells become self tolerant by undergoing secondary Ig gene rearrangements that replace an autoreactive B cell receptor with a non-autoreactive receptor (118). Replacing productively rearranged Lc or Hc genes can serve to eliminate production of an antibody that was autoreactive. Variable region of the Ig $\mathrm{H}$ and $\mathrm{L}$ chain $\left(\mathrm{V}_{\mathrm{H}} / \mathrm{V}_{\mathrm{L}}\right)$ regions determine the specificity of a receptor, and replacing either $\mathrm{V}_{\mathrm{H}}$ or $\mathrm{V}_{\mathrm{L}}$ can change receptor specificity (119-121).

Three articles in the same issue of the Journal of Experimental Medicine were the first to demonstrate receptor editing $(117,118,122)$. B cell hybridomas generated after LPS stimulation from the VH3H9 Hc only transgenic mice were analyzed for Lc by Radic et al. It was found that the Lc from the Vк12/13 gene family was observed most frequently and $\mathrm{J}_{\kappa 5} 5$ was used most of the time (122). This suggested that, certain light chains are used more frequently because they in association with the Hc do not bind to autoantigens. In another experiment, Gay et al generated transgenic mice $(\mathrm{H} / \mathrm{L})$ by introducing a cloned $\mathrm{V} \kappa 4 \mathrm{~J}_{\kappa} 4 \mathrm{C} \kappa$ (L only) gene segment in a $3 \mathrm{H} 9$ ( $\mathrm{H}$ only) transgenic mouse. The transgene was expressed in spleen and BM. B cells from spleen and BM were analyzed by flow cytometry with anti-idotypic antibodies named 1.209 and $1.3 \mathrm{H} 9$. 1.209 binds to $3 \mathrm{H} 9$ heavy chain and binds to $3 \mathrm{H} 9$ antibodies with a wide variety of light chains. $1.3 \mathrm{H} 9$ bound only to $3 \mathrm{H} 9+\mathrm{V} \kappa 4.1 .209$ bound to about $90 \%$ of adult splenic $\mathrm{IgM}^{+}$ cells from both $\mathrm{H}$ and $\mathrm{H} / \mathrm{L}$ transgenic animals. 1.3H9 did not bind any cells from the adult mice. This suggested that $3 \mathrm{H} 9 \mathrm{~V} \kappa 4$ cells were deleted in the animals. However the numbers of $\operatorname{IgM}^{+} \mathrm{B}$ cells in adult cells were normal. Moreover, spleen cells made into hybridomas did not produce antibody that had ANA patterns and also did not bind to DNA by ELISA. Primer extension analysis showed that the Hc was intact in these hybrids but there was expression of endogenous Lc rather than the transgenic Lc. Sequence analysis showed that only a limited set of Lc were expressed and members from the same group showed a high degree of clonal relatedness. This indicated that the autoreactive $3 \mathrm{H} 9+\mathrm{V} \kappa 4$ receptor is replaced by receptor editing (117).

\subsection{Regulation of receptor editing}

The discovery of receptor editing was followed by papers that attempted to explain this mechanism. Many reports have suggested that self antigen binding to the $\mathrm{BCR}$ of immature $\mathrm{B}$ cells in the BM stimulates receptor editing. Hertz and Nemazee tested this by using immature BM B cells and exposing them to BCR ligands. They showed that stimulating immature BM B cells with anti-idiotypic antibodies did not cause cell death, but instead the receptor cross linking caused an increase in RAG-2 mRNA levels and endogenous light chain gene rearrangements. The BM microenvironment 


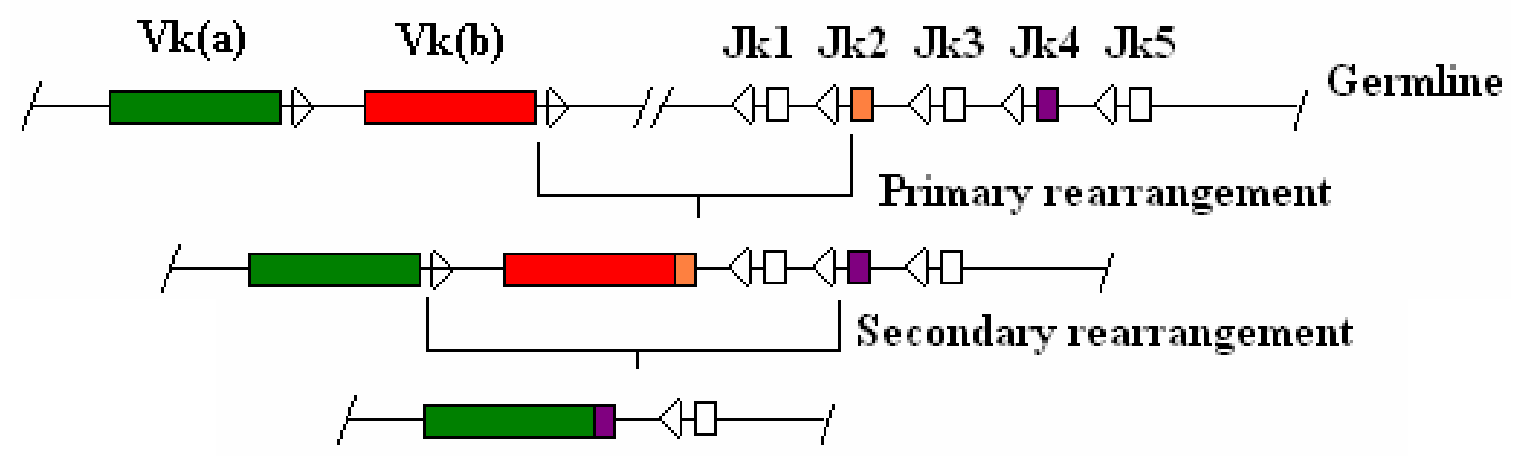

Figure 1.6 Receptor editing at the Lc locus. 
appeared to be essential for editing to occur because transfer of BM derived immature B cells into spleen cell cultures failed to show receptor editing. However if help was provided in the BM in the form of an anti CD-40 moclonal antibody, receptor editing was inhibited and B cells proliferated rapidly. This suggests that T-cell help can cause autoreactive B cells to escape tolerance mechanisms (59). Sandel and Monroe extended the observation that editing occurs in the context of BM environment and proposed that the site where the antigen is encountered first determines whether the cell undergoes receptor editing or deletion by apoptosis. They showed that cell to cell contact between $\mathrm{BM}$ and immature $\mathrm{B}$ cells protects them from BCR induced deletion and induced receptor editing (123).

It was observed that Lc replacement is the major mechanism by which receptor editing occurs. One of the papers that dealt with this phenomenon was by Prak et al. They used $3 \mathrm{H} 9$ transgenic mice that are $\kappa^{+/-}$(Only one functional $\kappa$ locus). $\kappa^{+/-} \mathrm{B}$ cells can survive by rearrangements either at the functional $\kappa$ locus or the $\lambda$ locus. It has been found that such mice have a higher $\lambda$ expressing B cells in the BM and the spleen. If $\kappa^{+/-}$ animals also have anti-DNA transgene then it becomes even more difficult for these cells to stave off autoreactivity. This in turn will cause more rearrangements at the available $\kappa$ locus and then at the $\lambda$ locus. $3 \mathrm{H} 9 \kappa^{+/-}$animals show a high percentage of cells that expressed $V \kappa 12 / 13$ and also had significantly higher rearrangements to $\mathrm{J}_{\kappa} 5$ (124). In $\kappa^{+/-}$ $3 \mathrm{H} 9$ Heavy chain transgenic mice, many $\mathrm{B}$ cells had the $\lambda \mathrm{x}$ light chain rather than $\lambda 1$ or $\lambda 2$ because $\lambda 1$ or $\lambda 2$ with $3 \mathrm{H} 9$ is autoreactive (120). The $V \lambda x$ is one of the three functional $\mathrm{V} \lambda$ genes in mice the other two being $\mathrm{V} \lambda 1$ and $\mathrm{V} \lambda 2$. The $\mathrm{V} \lambda \mathrm{x}$ Lc is considered an editor because this when combined with $3 \mathrm{H} 9$ or 56R Hc does not bind DNA (125).

In $3 \mathrm{H} 9 \kappa^{-/-}$mice, rearrangements to $\lambda \mathrm{x}$ is favored rather than to $\lambda 1$ or $\lambda 2$, and even when $\lambda 1$ is rearranged it often is non productive. $\mathrm{B}$ cells with productive $\mathrm{V} \lambda 1$ when present had the $3 \mathrm{H} 9 \mathrm{Hc}$ replaced by an endogenous Hc. Hc replacement is therefore another mechanism of receptor editing (126). Secondary rearrangements can continue until such time there are $\mathrm{J}_{\kappa}$ segments available but the rearrangements may or may not be productive. The B cell attempts to rectify its autoreactive receptor by secondary rearrangements at its $\kappa$ locus first and then if unsuccessful rearranges the gene segments at the $\lambda$ locus. Thus, after the production of an autoreactive $\operatorname{IgM}$ receptor (with a $\kappa \mathrm{L}$ chain) receptor editing can take three forms -1 ) recombination between an upstream $V \kappa$ to a downstream $\mathrm{J} \kappa$ on the same chromosome (55) 2) recombination can occur on the other chromosome 3 ) recombination can occur on the light chain $\lambda$ locus (124). V gene replacement by $\mathrm{H}$ chain is also observed (126).

\subsection{Allelic inclusion}

Receptor editing brings allelic inclusion with it. Allelic inclusion is the expression of two different productive Lc rearrangements. This has been shown in the $56 \mathrm{R}^{+}$ transgenic mice that express a $\kappa$ Lc and a $\lambda \operatorname{Lc}(127,128)$. Wabl and Gerdes demonstrated the presence of two $\kappa$ Lc in a nuclear transfer mouse (129). Nucleus from 
terminally differentiated B cells fron peripheral lymph node was injected into enucleated oocytes to derive cloned blastocysts and subsequently embryonic stem cells. The embryonic stem cells were injected into tetraploid blastocysts to generate nuclear transfer mice. In this method the placenta is derived from the tetraploid host cells and the embryo from the injected embryonic stem cells (130). Casellas et al. used gene targeting to replace the mouse IgK constant region $(\mathrm{mC \kappa})$ with its human counterpart $(\mathrm{hC} \kappa)$ and created transgenic mice. When B cells undergo receptor editing it is more easily identified by the presence of human $\kappa$ Lc constant region. They showed that $3-5 \%$ of $B$ lymphocytes express equal amounts of cell surface $\mathrm{mC} \kappa$ and $\mathrm{hC} \kappa$ light chains and that $10 \%$ of mature B cells coexpressed two Igk alleles. They suggest that these cells arise through receptor editing. They also demonstrate that B lymphocytes carrying two BCRs are recruited to germinal center reactions, and participate in humoral immune responses (131).

Like regular Ig gene rearrangements receptor editing is also due to random rearrangements and therefore could result in the production of BCRs that might be autoreactive (132). The idea of receptor editing is to rescue autoreactive B cells by replacing autoreactive BCRs. It was thought that expression of a new Lc leading to a new BCR halts the expression of the previously expressed Lc to dilute out the old BCR. However there are numerous examples in the literature now that this does not always happen. B cells have been shown to contain two productive rearrangements and also express two different receptors $(116,127,133)$. One area that still remains unclear is whether one receptor (probably non-autoreactive) takes up the normal functions while the other receptor (probably autoreactive) just remains inside. It is possible that the autoreactive receptor binds to an internal antigen while the other receptor carries out the normal duties of a cell surface BCR to move the B cell forward through its developmental stages and lead it to the peripheral lymphoid organs.

\subsection{BCR signaling threshold in B cell development and tolerance induction}

The BCR is the single most important component that is required for survival of $\mathrm{B}$ cells irrespective of what developmental stage they are. This is seen in mice which are either IgH or IgL knock-ins encoding the 3-83 BCR. The receptor in the absence of antigen unexpectedly showed $\mathrm{B}$ cells that underwent extensive receptor editing. It was proposed that insufficient receptor expression on the B cell surface may be responsible for the lack of developmental progression (134). Therefore, a certain level of receptor expression may be required to promote $\mathrm{B}$ cell maturation. The importance of continued expression of the BCR was demonstrated more dramatically in mature B cells by Rajewsky's group where they showed that removing surface Ig from mature B cells by cre/lox gene targeting kills the cell (135).

The BCRs must be present on the cell membrane for them to play a role in tolerance induction. This was shown in experiments that utilized the $3 \mathrm{H} 9+\mathrm{V} \kappa 4$ autoantibody of the IgG2a class. In this particular experiment, these antibodies had the secretory domain but did not have a transmembrane domain in its Hc. Therefore the 
IgG2a antibodies were secreted while endogenously expressed antibodies were both membrane bound and also secreted. Negative regulation of the secreted autoantibody is not effective and the sera from transgene positive animals showed elevated levels of antiDNA and anti-cardiolipin antibodies (136). When the Lc of the antibodies were analyzed it was found that the secreted-only IgG2a Hc paired with a wide range of L chains (121). This shows that for a receptor to be effective in maintaining tolerance it should be present in the proper context on the cell membrane.

The presence of BCR alone may not be sufficient for B cell survival if the receptor is unable to signal. Experiments have shown that basal signaling is necessary for $\mathrm{B}$ cell developmental progression at the immature stages. Interruption of basal IgM signaling in immature B cells, either by the inducible deletion of surface Ig via Cremediated excision or by incubating cells with the tyrosine kinase inhibitor herbimycin A or the phosphatidylinositol 3-kinase inhibitor wortmannin, led to a "backdifferentiation" of cells to an earlier stage in B cell development. This was characterized by the expression of pro-B cell genes. Cells undergoing this reversal in development also showed evidence of new LC gene rearrangements, suggesting an important role for basal Ig signaling in the maintenance of light chain allelic exclusion (137).

A basal level of signaling can be maintained by Hc alone. However, it has been shown that the surrogate Lc (SLC) is required for assembly of the Hc. Both the Hc and $\lambda 5$ (a component of the SLC) must be present during B cell development because mutations preventing the expression of either the Ig Hc or $\lambda 5$ results in developmental arrest (43). To understand the precise roles of the various components of the pre-BCR, Shaffer and Schlissel used mice transgenic for a surface-expressed, but truncated, form of heavy chain that cannot associate with surrogate light chains, to study the role of SLC in $\mathrm{B}$ cell development. The truncated $\mathrm{Hc}$ lacked the $\mathrm{VH}$ and $\mathrm{CH} 1$ domains and yet escaped ER retention to be expressed on the cell surface. They found that expression of the truncated heavy chain transgene did not result in changes in surface marker expression, germline $\kappa$ locus transcription, and V(D)J recombinase. Therefore they concluded that surrogate light chains, are necessary for the assembly of the wild-type pre-BCR, but are not directly involved in pre-BCR signaling and are not required for early B cell development (138).

Lang et al wanted to test if the affinity of an autoantigen to its receptor has any bearing on B cell tolerance. 3-83 antibody is anti-MHC I. MHC I alloforms that had very low but detectable affinity were identified. Mice expressing these low affinity autoantigens were crossed with 3-83 expressing mice. It was shown that the low affinity antigens induced effective receptor editing and also autoreactive B cells were absent in the periphery. This showed that immature B cells might be very sensitive to even low affinity self antigens in the BM (139).

The role of quantity of self antigen required for regulating autoreactive $\mathrm{B}$ cells was studied in a HEL-anti HEL system. A homogeneous population of Anti-HEL antibody expressing $B$ cells was allowed to exist in the presence of different numbers of cells that express HEL in irradiated RAG2 $2^{-/}$mice. HEL antibody specific B cells were 
increased in the periphery when the HEL expressing cells were rare. If the HEL expressing cells were very low then normal anti-HEL B cell numbers were observed in the periphery. B cells expressing low levels of surface receptor in the presence of B cells secreting very low levels of autoantigen were shown to be positively selected (140).

\subsection{B cells may be positively selected}

Role of negative selection for B2 B cell repertoire is well documented but the evidence for positive selection is mostly indirect. If a $\mathrm{B}$ cell is unable to make a receptor at any developmental stage, or if it is unable to transmit signals intracellularly, it is blocked from further differentiation. This is considered as evidence for positive selection. The nature of this positive selection signal is unclear. Loder et al, show that in CD45 and Btk mutant mice the B cells are arrested at the T2 cell stage in the spleen and in Ig $\alpha$ cytoplasmic tail mutants (signaling defective) the development of T1, T2, MZ and mature $\mathrm{B}$ cells is affected causing a decrease in cell numbers (141). Monroe and colleagues have demonstrated that just the cytoplasmic domains of $\operatorname{Ig} \alpha$ and $\operatorname{Ig} \beta$ in the absence of BCR are sufficient for $\mathrm{B}$ cell differentiation to a splenic $\mathrm{B}$ cell. This argues that positive selection at the pre- $\mathrm{B}$ and immature $\mathrm{B}$ cell stages may require only tonic signaling from the preBCR and BCR (142). Schlomchik at al., looked at differences in the BCR repertoire of immature and mature cells by flow cytometry and cloned PCR products into a library for screening. They found that in-frame $\kappa$ repertoire of immature $B$ cells was diverse but in

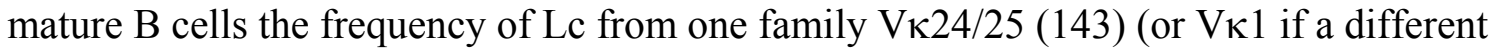
mice strain was used) was significantly enhanced. Since only a few H/L pairs are enriched in the mature $\mathrm{B}$ cell population they consider this to be proof for positive selection (144). Cyster et al., showed that immature anti-HEL B cells lacking CD45, despite their signaling handicap, go on to become mature B cells if HEL is available during development (145). Wang and Clarke used the VH12 B cells that either bind or do not bind to phosphatidylcholine (PtC) to show that $\mathrm{PtC}$ non binders 6-1/Vк1A B cells are present as transitional B cells but do not differentiate further. They interpret this developmental arrest as evidence for a ligand mediated positive signal (146).

\section{$1.13 \mathrm{~B}$ cells in the periphery}

Immature $\mathrm{B}$ cells exit the BM and migrate to the spleen and lymph nodes. For example, B cells can be present in the follicles or the MZ in the spleen. MZ B cells are interesting because of their association with autoreactivity and also their ability to react quickly to bacterial antigens.

MZ B cells constitute only $5-10 \%$ of total splenic B cells. The accessibility of MZ B cells $\left(\operatorname{IgM}^{\text {high }} \operatorname{IgD}{ }^{\text {low }} \mathrm{CD} 21^{\text {high }} \mathrm{CD} 23^{\text {low }}\right.$ ) to the bloodstream allows this subset to initiate rapid $\mathrm{T}$-independent IgM responses to blood-borne antigens. In contrast, follicular $\mathrm{B}$ (FO B) cells ( $\left(\mathrm{IgM}^{\text {low }} \mathrm{IgD}{ }^{\text {high }} \mathrm{CD} 21^{\text {int }} \mathrm{CD} 23^{\text {high }}\right.$ ) reside in the splenic follicle bordering the periarteriolar $\mathrm{T}$ cell zone, a position that facilitates interaction with $\mathrm{T}$ cells and participation in T-dependent immune responses. The MZ B cells differentiate quickly 
into IgM producing plasma cells or sometimes function as antigen presenting cells (147). The absence of the MZ in humans in early life coincides with susceptibility to blood borne pathogens (148). These cells exist in a partially activated state and also express high levels of $\operatorname{IgM}$ that facilitate high avidity interactions with repetitive capsular antigens. MZ can also secrete class switched antibodies efficiently (149). Once activated, MZ B cells differentiate into plasma cells within the extrafollicular compartments of secondary lymphoid organs (147). The MZ B cell IgM response can be observed in vitro using the bacterial component LPS as a stimulant. LPS treated MZ B cells respond rapidly, exhibiting robust $\mathrm{Ab}$ secretion, proliferation, and up-regulation of costimulatory molecules (150). LPS treatment of FO B cells induces these same events, but they do not occur as quickly or as strongly as in MZ B cells (151). It has been reported that a molecule named Notch-2 is important for B cells to become residents of the MZ (152, 153). Lower levels of another transcription factor named E2A also results in MZ B cell phenotype (154). Others have reported that strength of signaling through the BCR is responsible for mature B cells becoming either the MZ B cells or follicular B cells. The signal strength model has been proposed in the context of a transcription factor named Aiolos and another kinase, the Bruton's tyrosine kinase. In this model, strong signaling through the BCR leads to development of follicular B cells whereas weak signaling through the BCR results in MZ B cell formation (155). Rajewsky's group used the Epstein Barr virus protein LMP2A as a BCR surrogate in BCR deficient mice. LMP2A has an immunoreceptor tyrosine-based activation motif similar to that found in the $\operatorname{Ig} \alpha$ and $\operatorname{Ig} \beta$ signaling subunits of the BCR, in its $\mathrm{N}$-terminal intracellular region (156). LMP2A protein may sequester Src family protein tyrosine kinases and Syk from the BCR signaling complex and inhibit BCR-induced intracellular calcium mobilization (157), providing the infected B cells with a BCR-independent survival signal at the same time. LMP2A expressed under a strong or weak promoter leading to high or low receptor expression. Low expression presumably leads to low signal strength and B cells in this case migrate to the MZ (158). These results are in direct contrast to the observations by Kanayama et al. This group made a quasimonoclonal Hc transgenic mice that express BCRs having different affinities for nitrophenyl acetyl in association with different light chains. They found that receptors that had a higher affinity for the antigen were present in the MZ. The MZ B cell development also has been proposed exclusively based on BCR density or based on multi reactivity of the receptor (159). Moreover, it has been proposed that constraints during the developing stages of B cells (160) or just the presence of multireactive receptors (11) on B cells lead to MZ development. Also, there is no easy way to determine if and why cells that are in the MZ migrate to the follicle or the vice versa.

\subsection{Check points to prevent autoreactivity}

The actual number of autoreactive B cells that arise during B cell development is not known and also when and where these B cells are regulated is not clear. To get a better idea about these questions Wardemann et al., cloned antibodies from single B cells derived from the BM and blood. Early immature B cells and immature B cells were analyzed. Cells that had a surface phenotype of pre-B cells (but express Igא or Ig $\lambda$ ) were 
designated early immature B cells $\left(\mathrm{CD} 34^{-} \mathrm{CD} 19^{+} \mathrm{CD} 10^{+} \mathrm{IgM} \mathrm{M}^{-}\right)$. Immature $\mathrm{B}$ cells were cells that expressed surface $\operatorname{IgM}\left(\mathrm{CD} 34^{-} \mathrm{CD} 19^{+} \mathrm{CD} 10^{+} \operatorname{IgM}^{+}\right)$. It was shown that about $76 \%$ of the antibodies from early immature B cells had high reactivity in HEp-2 ELISAs. The frequency of reactive antibodies from the immature B cells stage dropped to about $43 \%$ but remained at about $40 \%$ in newly emigrated B cells $\left(\mathrm{CD} 27^{-} \mathrm{CD} 19^{+} \mathrm{CD} 10^{+} \operatorname{IgM}^{+}\right)$ found in the blood. Further reduction of autoantibodies was noticed in mature naïve $\mathrm{B}$ cell clones $\left(\mathrm{CD} 27^{-} \mathrm{CD} 19^{+} \mathrm{CD} 10^{-} \mathrm{IgM}{ }^{+}\right)$. They concluded that most self-reactive B cells are removed at the immature $\mathrm{B}$ cell stage in the $\mathrm{BM}$ and also transition stage from the newly migrated to the mature naïve B cell stages (107). Yurasov et al., later showed that in SLE patients autoreactive antibodies were not effectively removed at the transition between new emigrant $\left(\mathrm{CD} 19^{+} \mathrm{CD} 10^{+} \mathrm{IgM}^{+} \mathrm{CD} 27^{-}\right)$and mature naïve $\mathrm{B}$ cells $\left(\mathrm{CD} 19^{+} \mathrm{CD} 10^{-}\right.$ $\left.\operatorname{IgM}^{+} \mathrm{CD} 27^{-}\right)(161)$. The autoantibodies were polyreactive and in one case even increased in the mature naïve B cells in a SLE patient. Therefore it was concluded that SLE patients have a significant defect in the early B cell tolerance ckeckpoint between the BM and the periphery (162).

\subsection{Autoimmunity}

Autoimmunity is an immune response to "self". Although many cell types contribute to autoimmune disorders, lymphocytes are key effectors for the initiation and propagation of autoimmunity (163). Autoimmunity results from failed establishment and maintenance of tolerance to self-antigens. Immunological tolerance is the ability of the individual to differentiate "self "from "non-self". Normally the immune system does not attack components that make up the organism. The abnormal autoantibody responses are similar to the normal immune responses and use B and T-cell genes. B-T cooperation is essential for autoantibody responses. $\mathrm{T}$ lymphocytes are activated when their receptors bind to antigen presented by an antigen presenting cell (Apc). B cells and macrophages can function as Apc. Autoimmunity can be systemic or organ specific. Systemic autoimmunity is autoreactivity that is not restricted to a specific organ. The main diseases under this group are SLE and chronic graft-versus-host disease (cGVH) among many others. The presumption that a disease is autoimmune is mainly based on the presence of autoantibodies. Autoimmune disease differs from person to person and the disease can flare up and subside periodically (164). Some patients display attributes of multiple systemic autoimmune disorders.

SLE, an important systemic autoimmune disease, is characterized by the presence of autoantibodies against DNA and/or nucleosomes. The anti-nuclear antibodies (ANAs) form immune complexes with free DNA present in the body (165). It is thought that these complexes accumulate in the kidney glomeruli (166) and joints to initiate a type III hypersensitivity reaction that manifests as glomerulonephritis, arthritis, vasculitis and various other possible SLE-associated clinical findings (167). Free DNA and histones at sites of high cell turnover are cleared by DNase I, a major nuclease present in serum. Therefore in DNase I knockout mice there are classical symptoms of SLE like glomerulonephritis (165). It has also been shown that serum DNase I levels are lower in SLE patients (168). Anti-DNA antibodies in SLE patients are directed mainly against 
dsDNA. dsDNA antibodies recognize DNA base-pairs rather than the backbone. Antibodies against all histone classes occur in SLE. A main target of anti-histone antibodies is the H2A-H2B DNA complex (169). Anti-histone antibodies in immunofluorescence assays show homogenous chromosomal staining in dividing cells. Some SLE patients exhibit anti-phospholipid antibodies. Cardiolipin and phosphatidylserine are the preferred antigens (169). Binding to phospholipids appears to be dependent on a serum cofactor called $\beta 2$-GPI (170). Antinuclear antibodies often show binding to multiple antigens. It was suggested that multi-reactivity may either be due to changes in conformation of the antibody combining site or the combining site has different areas each capable of binding to a different antigen. Anti-DNA antibodies binding to nucleosomes may be called multi-reactive because one part of the antibody combining site may interact with the DNA and the other with the protein histones. AntiDNA antibodies often have mutations to amino acids arginine (R) or asparagine $(\mathrm{N})$ which are residues that favor dsDNA binding. Anti-histone antibodies acquire mutations to aspartic acid (D) or glutamic acid (E). Thus, negatively charged residues in the antibody combining sites are thought to bind to the positively charged lysine $(\mathrm{K})$ and arginine $(\mathrm{R})$ in the extended portion of the histones. Anti dsDNA antibodies belonging to the IgG class cause more severe disease symptoms and tissue damage than IgM antibodies because IgG arise after SHM and affinity maturation $(171,172)$ and are more specific to tissue autoantigens.

The presence of Ig in serum or urine prompts the consideration of multiple myeloma, Waldenstrom's macroglobulinemia and the relatively common and asymptomatic monoclonal gammopathy of undetermined significance (MGUS). Even a small B cell clone, as seen in MGUS, may synthesize a harmful monoclonal protein that can cause devastating systemic organ damage and dysfunction through its aggregation and deposition or through its antibody activity against autoantigens (173).

In transgenic mice models, increased B cell activation leads to autoantibodies and systemic autoimmunity as demonstrated when CD19, a costimulatory signaling component associated with the BCR, was overexpressed. CD19 overexpression leads to autoantibody production probably due to a lower threshold of B cell activation (174, $175)$. Genes that constitutively down regulate $B$ cell function, appear to play a part in preventing autoimmunity. Therefore animals deficient in or lacking CD22 (176), TGF $\beta$, CD45, Fc $\gamma$ RIIb (177) and SHP-1 (178) suffer from progressive autoimmune disease. IgM antibodies to DNA are produced when B cells are activated by LPS leading to some degree of systemic autoimmunity. It is the MZ B cells that are activated first by LPS and it has been suggested that autoreactive B cells reach the MZ. LPS is the major component of Gram negative bacteria. This might be a good model for autoimmunity that arises due to infections (179).

\subsection{Autoantibody characteristics}

Anti-DNA antibodies in individuals are usually clonally related which implies that autoantibody generation is "antigen-driven" and involves clonal expansion. In 
general, autoantibodies are produced after a process of SHM (180). Autoantibodies from patients with systemic diseases usually bind with greater avidity to antigens from the same species. This implies that autoantibody generation is self-antigen dependent and comes about after affinity maturation. Epitope mapping studies have shown that autoantibodies recognize multiple epitopes of nuclear proteins (181). The anti nuclear antibodies are directed mainly against dsDNA and histones (182). Anti-dsDNA antibodies bind nucleosomes also (183). Moreover, anti-histone antibodies bind mainly to $\mathrm{H} 1$ and H2B (184), the most accessible histones in the nucleosome particle which shows that anti-Histone antibodies also target nucleosomes (185). Nucleosomes are released by hybridoma cells undergoing apoptosis (186) and are also exposed at the cell surface of apoptotic cells (187). Immune complexes form between anti-nuclear antibodies and nucleosomes and get deposited in the glomerular basement membrane in lupus.

Many sera with antibodies to cytoplasmic antigens may be reported as 'antinuclear antibody (ANA) negative', if the antibodies have specificity for cytoplasmic auto antigens (188). ANA negative sera do contain autoantibodies that react with cytoplasmic antigens such as the Golgi complex, centrosomes (189), lysosomes, and proteasomes (169).

\subsection{Anti-Golgi autoantibodies}

Anti-Golgi antibodies (AGA) were first identified from a patient with Sjogren's syndrome with lymphoma (190). Subsequently AGA have been reported in SLE (191), Raynaud's phenomenon (192), Wegener's granulomatosis (193) and viral infections including Epstein Barr virus (194), Hepatitis B (195) and HIV (192). Representative human serum containing AGA were used to screen a HEpG2 expression library and the purified AGA from the serum recreated the Golgi pattern of immunofluorescence on HEp-2 cells. The AGA did not have reactivity to microtubules, nucleus or the plasma membrane. Patient sera react with Golgi autoantigens that range from 35 to 260kDa (191, 196) as seen by immunoblotting studies. However, AGA antibodies are predominantly against five Golgi autoantigens which are giantin, golgin-95, golgin-97, golgin-160 and golgin-245. The Golgins have $\alpha$-helical coiled coils except for the $\mathrm{N}$ - and $\mathrm{C}$ - terminal domains. Giantin is the most common autoantigen against which AGA were present. Incidentally giantin is also the highest molecular weight Golgi antigen. Epitope mapping of giantin using recombinant fragments showed that epitopes at the $\mathrm{C}$-terminal transmembrane domain on the cytoplasmic face of the Golgi were the most reactive to AGA in the sera (197). Usually the human sera react to only one autoantigen which suggests that the immune response is not directed only to the coiled coils. This view is reinforced because AGAs do not react against other coiled coil domain containing autoantigens such as nuclear mitotic apparatus protein (198), lamin B (199), myosin Hc (200), SS-A/Ro (201) and Ku (202). It is thought that the Golgi autoantigens may be released into the immune system after cell lysis which could be responsible for stimulating the production of AGA (197). 


\subsection{Autoantigens}

Nuclear protein complexes arguably are the most important autoantigens. Components of the nucleus particularly chromatin and RNA associated proteins are recognized as autoantigens. Nucleosome, the fundamental unit of chromatin likely plays a key role in autoimmune diseases like SLE. Two copies each of histones H2A, H2B, H3 and H4 make up the nucleosome core particle around which helical DNA made up of 146 base pairs is wrapped. Adjacent nucleosome particles are linked like beads on a string by histone free DNA and a molecule of histone $\mathrm{H} 1$ is present at the point where the DNA starts wrapping the core histone particle (182).

Most proteins are post-translationally modified. However some proteins with post-translational modifications function as SLE autoantigens (203). RA patients show antibodies to citrullinated proteins in the inflamed synovium (204). Likewise SLE associated nuclear autoantigens get modified or cleaved during apoptosis (205). The presence of antigen is required for autoimmunity to develop but other factors likely play a role. For example, injecting mice with apoptotic bodies results in the production of a low level of anti-nuclear antibodies and the response is muted and short lived (206). Injecting unaltered DNA does not provoke an autoimmune response. However, when it is coupled to other proteins or if the DNA strands are damaged then there is a response (207). Interestingly it has been shown that there is a small amount of circulating DNA conjugated to histones in SLE patients (208). It has been proposed that apoptotic blebs may harbor autoantigens (209). However, it appears that, for blebs to be effective autoantigens, clearance of apoptotic cell debris by macrophages must be defective (210). Apoptotic blebs might serve as autoantigens in the presence of infectious agents, as shown by generation of T-cell responses and secretion of inflammatory cytokines by macrophages upon injection of apoptotic bodies plus LPS (211).

\subsection{Importance and purpose of this study}

The current knowledge about $\mathrm{B}$ cell regulatory mechanisms has been derived from transgenic mice producing B cells against either non-disease associated antigens or DNA. In the case of non-disease associated antigens, though we can control the amount, type and location of antigen, this still does not permit us to study B cell regulation as it would occur normally. With anti-DNA transgenic mice, there is the assumption that developing B cells encounter DNA in the BM. In what form is the DNA and how strong the binding between the BCR and the DNA is not known. Therefore to study these aspects of $\mathrm{B}$ cell regulation we made recombinant receptors and analyzed their binding properties to disease associated autoantigens. Moreover, in spite of the B cell tolerance mechanisms and various checkpoints it is not clear how autoreactive B cells break tolerance and get activated to produce autoantibodies in the periphery. While studying the receptor properties, we came across an interesting anti-DNA receptor that led us to propose a new mechanism for B cells to escape tolerance mechanisms and migrate to the periphery. These findings are dealt with and discussed in the next chapter. 
One important criterion that defines SLE is the detection of autoantibodies in the patient serum. Though the titres of autoantibodies can be assessed, it is not clear which autoantibodies are responsible for disease. In fact, it was recently shown that in spite of having substantial serum anti-DNA antibodies, noticeable clinical disease was limited in mice (212) that were B cell activating factor (BAFF) deficient. These data suggests that anti-DNA antibodies may not be solely responsible for autoimmune disease. Also, the exact $\mathrm{B}$ cells that produce autoantibodies cannot be identified and where they reside in the periphery is not clear. Autoreactive B cells must be activated by antigen because IgG are involved in pathogenesis and the B cells undergo SHM (172). Though it is considered in general that anti-DNA antibodies are responsible for pathogenesis in SLE it is not clear which autoantibodies are precisely responsible for disease. SLE is defined by various types of glomerulonephritis and mostly all of them are due to deposition of immune complexes on the glomerular basement membrane. Do the autoantibodies form an immune complex while in the serum and then get deposited at the basement membrane? This scenario appears to be unlikely because immune complexes are not readily detectable in SLE patients $(213,214)$. More recently it was shown that anti-nucleosomal antibodies binding to histones of nucleosomes get deposited more readily on the heparan sulfate present in the glomerular basement membrane (166). Clearly, heparan sulfate cannot be considered an autoantigen because the anti-nucleosomal antibodies do not bind to it directly. Therefore, the target antigen in the glomerular basement membrane for the autoantibodies has not been demonstrated conclusively.

In addition to SLE, Ig deposition diseases are another class of diseases where antibodies or components of an antibody are deposited in tissues. Either Hc or Lc can be exclusively deposited in tissues to cause pathogenesis. Lc deposition disease is referred to as primary amyloidosis. Here, insoluble Lc fragments are produced that polymerize into fibrils and get deposited extracellularly causing visceral organ dysfunction. Amyloidosis is associated with nephritis, cardiomyopathy, neuropathy and hepatomegaly (215). In addition, there are deposits that consist of apparently normal HL pairs (216). Why do certain cells make antibodies that get deposited? Are these antibodies autoreactive? Why do Lc from certain cells gets secreted in higher quantities? There is no definite answer to any of these questions yet. Our studies uncovered a B cell that secretes antibody potentially_capable of tissue deposition. Characteristics of these B cells and the autoantibody they secrete are dealt in detail in the third chapter of this thesis. 


\section{CHAPTER 2: EDITING AND ESCAPE FROM EDITING IN ANTI-DNA B CELLS*}

\subsection{Introduction}

Receptor editing replaces autoreactive BCRs with non-autoreactive BCR (217, 218). It is accomplished through secondary rearrangement of upstream Ig light (L) (118, $122,219)$ or heavy $(\mathrm{H})$ chain $(126)$ variable $(\mathrm{V})$ genes. Autoreactive B cells that would otherwise be deleted $(220,221)$ use editing to revise their receptors and exit from the BM. Given the high frequency of autoreactivity among immature B cells (107), receptor editing plays a major role in determining the size and shape of the mature B cell repertoire $(131,222)$.

This mechanism also yields incompletely or partially edited B cells. Incompletely edited BCR retain some self-reactivity and/or gain new autospecificities. B cells expressing such BCR are a liability as they are activated in lupus susceptible mice and during induction of lupus $(119,127,223,224)$. They can evolve to pathogenic effector cells by mutation and class switch. This pathway to autoimmunity is illustrated by $3 \mathrm{H} 9$, a typical pathogenic anti-DNA expressed by a diseased mouse (114). By reverting the somatically mutated $\mathrm{VH} 3 \mathrm{H} 9$ to its germline sequence, it was discovered that the precursor of $3 \mathrm{H} 9$ bound to phosphatidylserine (PS) (170). Subsequent mutations gave rise to $3 \mathrm{H} 9$, an $\mathrm{Ab}$ with reactivity toward dsDNA, nucleosomes, and additional phospholipids $(115,120,225,226)$. Thus, an incompletely edited B cell was the precursor of this pathogenic autoAb.

Partial editing leads to co-expression of $\mathrm{L}$ chains, one of which is autoreactive. The autoreactive $\mathrm{H} / \mathrm{L}$ combination thus gains access to the periphery. It escapes central tolerance, we presume, because the non-autoreactive $\mathrm{H} / \mathrm{L}$ pair dilutes the expression of the autoreactive pair. Since we discovered this type of partially edited B cell, many examples of allelic or isotypic inclusion have been observed (116, 127, 128, 227, 228), including additional tg-encoded autoreactivities $(229,230)$, and autoreactivities present within an unperturbed repertoire $(129,131)$. Such B cells have been referred to as "Trojan horses" and may, if activated in the periphery, pose a risk of autoimmunity.

Here we describe an incompletely edited anti-dsDNA B cell that is regulated in a novel way: This B cell retains anti-dsDNA reactivity and acquires specifity for a Golgiassociated antigen. Its antibodies accumulate in the cell interior, bound to the Golgi apparatus.

\footnotetext{
*Adapted with permission from PNAS. Salar N. Khan, Esther J. Witsch, Noah G. Goodman, Anil K. Panigrahi, Ching Chen, Yufei Jiang, Amy M. Cline, Jan Erikson, Martin Weigert, Eline T. Luning Prak, and Marko Radic. 2008. Editing and escape from editing in anti-DNA B cells. PNAS. March 11, 2008, Vol 105, No:10, 3861-3866.
} 
The most direct interpretation of our results argues that the altered site of BCR expression allows these B cells to escape central tolerance and accumulate in the periphery. This mechanism may conceal a partially edited or unedited autoantibody expressing B cell.

\subsection{Methods}

\subsubsection{Construction of bivalent variable fragments}

Our system for bivalent scFv expression in E.coli and the $\mathrm{H}$ chain variants of $3 \mathrm{H} 9$ were previously described. We cloned the murine Vк12/13A (referred to as 12-46 by Thiebe et al.) as a rearrangement to $\mathrm{J}_{\kappa} 2$ from the Balb/c hybridoma $7942-\mathrm{H} 40$ by using the oligonucleotide primers Vк12/13 amino (5'-GCG TGT GGA TCC GAC ATC CAG ATG ACT CAG-3') and Jא2 (5'-GCG GTT AGA TCT CCA GCT TGG TCC CCC CT3'). The primers introduced unique restriction sites at the amino and carboxy termini of the $\mathrm{V}_{\mathrm{L}}$ domain that were complementary to sites in our expression vector. The $\mathrm{V} \kappa 21 \mathrm{D}$ (21-4; Thiebe et al.) to $\mathrm{J} \kappa 2$ and $\mathrm{V} \kappa 38 \mathrm{c}$ (gj38c; Thiebe et al.) to $\mathrm{J} \kappa 1$ rearrangements were similarly amplified from hybridoma DNA (22) by using primers Vк21D amino (5'-GCG

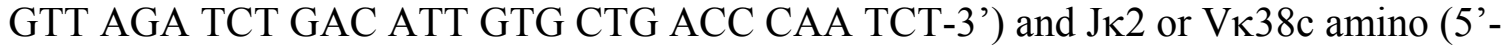
GCG TGT GGA TCC GAC ATC CAG ATG ACA CAG-3') and Jк1 (5'-GCG GTT AGA TCT CCA GCT TGG TGC CTC CA-3') respectively. In this manner, nine H/L chain combinations were constructed. Each $\mathrm{scFv}$ was fused in-frame to the c-jun leucine zipper to generate bivalent Fv that contained the domain B of Staphylococcal protein A for detection and a carboxy terminal His-tag for purification of the fusion protein.

\subsubsection{Bacterial expression and purification of $\mathrm{scFv}$}

Rosetta cells (Novagen, San Diego, CA) were transformed with scFv expression vectors and grown as previously described. Crude proteins were purified from the bacterial periplasm using Ni-NTA agarose chromatography (Qiagen,Valencia, CA), washed twice and eluted in $0.05 \mathrm{M}$ Tris- $\mathrm{HCl}, 1 \mathrm{M} \mathrm{NaCl}$ and $0.3 \mathrm{M}$ imidazole, $\mathrm{pH}=8.0$. The eluate was dialyzed against Bicine-EDTA buffer (50 mM Bicine $\mathrm{pH}$ 8.0, $0.15 \mathrm{M}$ $\mathrm{NaCl}$ and $10 \mathrm{mM}$ EDTA) prior to use in binding assays. To demonstrate the relative purity, size, and standardized concentration of the scFv, $1 \mu \mathrm{g}$ of each protein was analyzed by SDS-PAGE. The recombinant proteins migrated close to $40 \mathrm{kD}$, as predicted for our $\mathrm{scFv}$ fusion protein monomers. The small differences between the $\mathrm{scFv}$ were expected due to size differences between the $\mathrm{V} \kappa$ used in $\mathrm{scFv}$ construction.

\subsubsection{Binding assays}

The recombinant $\mathrm{Ab}$ fragments were used in an antigen capture assay (115), whereas PS binding was assessed by direct immunoassay (170). 


\subsubsection{Cell culture}

Jurkat T cells (Clone E6-1 ATCC, Manassas, VA) were used to assess scFv binding (187, 231). B cell hybrids expressing VH56R+Vк38c IgM (Hybridoma 160.3, and VH56R+Vк21D IgM (Hybridoma 79.1) were derived from a VH56R mouse with

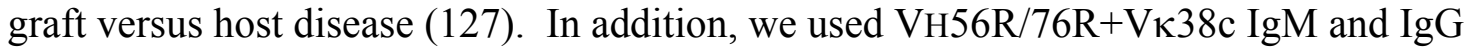

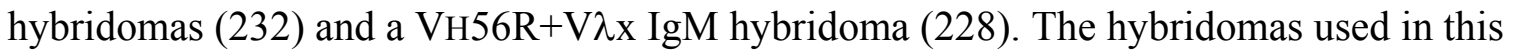
study were analyzed by RT-PCR and ELISA to minimize the possibility of allelic and isotypic inclusion $(127,228)$

\subsubsection{Immunofluorescence and confocal microscopy}

Cells were fixed with $6 \%$ paraformaldehyde for 15 minutes on ice and permeabilized with $0.075 \%$ Triton-X. To identify the Golgi, AF488-labeled lectin II (Invitrogen, Carlsbad, CA) was used. The IgM was visualized with AF647 labeled goat anti-mouse IgM (Invitrogen), and DNA was stained with Sytox Orange (Invitrogen). Surface IgM was visualized by staining live cells on ice and in the presence of sodium azide. Bound $\mathrm{scFv}$ were detected with AF647-conjugated rabbit anti-mouse IgG (Invitrogen). Samples were viewed on a Zeiss LSM510 laser scanning microscope, as described (187).

\subsubsection{Animals}

The VH56R Tg has been described previously $(116,172)$. VH56R mice were backcrossed on the C57BL/6 background for ten generations.

\subsubsection{Flow cytometry}

Splenocytes were harvested from VH56R mice and littermate controls, washed, and resuspended at $1 \times 10^{7}$ cells/mL for staining with the following Abs: FITC CD21 (Clone 7G6), PE CD23 (Clone B3B4), Biotin IgMa (Clone DS-1), all from BD Pharmingen (San Jose, CA), APC AA4.1 and B220 APC-Cy7 from eBiosciences (San Diego, CA), and our own anti-IgM a antibody, RS 3.1, conjugated to AF 488. Stained samples were analyzed and sorted on a FACSVantage SE Cell Sorter (BD Biosciences). Flow cytometric data were analyzed using FlowJo version 6.4.7 software (TreeStar, Ashland OR). To examine intracellular IgM in B cells, splenocytes from one VH56R mouse were sorted for IgM $\mathrm{I}^{\mathrm{a}}$-low surface phenotype and cultured in RPMI media with $10 \% \mathrm{FCS}, 10 \mu \mathrm{M} \beta-\mathrm{ME}$, and antibiotics for $48 \mathrm{~h}$. At the end of that period, cells were harvested and prepared for confocal microscopy, as described above. 


\subsubsection{Quantitative PCR}

Genomic DNA was purified from sorted cells and used for quantitative PCR $\left(40^{\circ} \mathrm{C} 10 \mathrm{~min} ., 95^{\circ} \mathrm{C} 10 \mathrm{~min}\right.$., followed by 60 cycles at $95^{\circ} \mathrm{C} 10 \mathrm{sec} ., 60^{\circ} \mathrm{C} 30 \mathrm{sec} ., 72^{\circ} \mathrm{C} 1$ sec.), as suggested for the LightCycler 480 real-time PCR system (Roche Applied

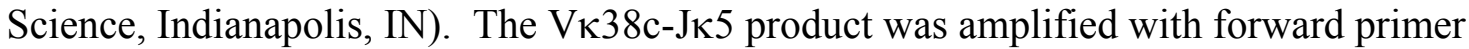
(5'-CTC ATA CAT TAC ACA TCT ACA TTA CAG CC-3') and reverse primer (5'TGC CAC GTC AAC TGA TAA TGA GCC CTC TC-3') and detected with a FAMlabeled hydrolysis probe (5-TCC TTC AGC ATC AGC AAC CTG-3'). For each sample, the $\mathrm{V} \kappa 38 \mathrm{c}-\mathrm{J}_{\kappa} 5$ product was normalized to a $\beta$-actin PCR product and compared to wildtype C57BL/6 B220 ${ }^{+} \operatorname{IgM}^{+} \kappa^{+}$splenocytes.

\subsubsection{Single cell sorting, cDNA synthesis and PCR}

$\operatorname{IgM}^{\mathrm{a}}$-bright $\left(\mathrm{CD} 19^{+} \operatorname{IgM}\right.$ allotype $\mathrm{b}^{-} \operatorname{IgM}$ allotype $\left.\mathrm{a}^{\text {high }}\right), \operatorname{IgM}^{\mathrm{a}}$-dim $\left(\mathrm{CD} 19^{+} \operatorname{IgM}\right.$ allotype $\mathrm{b}^{-} \mathrm{IgM}$ allotype $\left.\mathrm{a}^{\text {low }}\right), \mathrm{MZ}\left(\mathrm{B} 220^{+} \mathrm{CD} 21^{\text {hi }} \mathrm{CD} 23^{\text {int }}\right)$, and Fo B cells $\left(\mathrm{B} 220^{+} \mathrm{CD} 21^{\mathrm{int}} \mathrm{CD} 23^{\mathrm{hi}}\right.$ ) from one VH56R/B6 mouse were sorted and Ig gene rearrangements were assayed using primers for $\mathrm{VH} 56 \mathrm{R}, \mathrm{V \kappa} 38 \mathrm{c}$, and a degenerate $\mathrm{V} \kappa(\mathrm{s})$ primer with nested $\mathrm{C} \kappa$ reverse primers, as previously described $(127,233)$. We sequenced $76 \mathrm{V \kappa 38c-positive} \mathrm{PCR} \mathrm{products} \mathrm{from} \mathrm{individual} \mathrm{wells} \mathrm{in} \mathrm{five} \mathrm{96-well} \mathrm{plates.}$ Of these, 75 sequences matched $V \kappa 38 \mathrm{c}$ in-frame rearrangements.

\subsection{Results}

We have recreated the splenic $\mathrm{B}$ cell repertoires of three $\mathrm{H}$ chain tg mice (Figure 2.1) in vitro and observed that $\mathrm{VH} / \mathrm{VL}$ pairs expressed in the spleen do not bind to dsDNA. However, we also discovered an exception: VH/VL pairs incorporating the editor $\mathrm{V} \kappa 38 \mathrm{c}$ retained affinity for dsDNA yet were expressed in the spleen. To explore why central tolerance is unable to regulate these anti-dsDNA VH/VL pairs, we examined their cellular distribution in hybridomas and their expression in splenic B cell subsets.

\subsubsection{Analysis of autoreactivity}

The repertoire in anti-dsDNA $\mathrm{H}$ chain tg mice is highly biased toward editor $\mathrm{L}$ chains (Figure 2.2). The IgM antibodies encoded by the $\operatorname{tg} \mathrm{H}$ and editor $\mathrm{L}$ chains bind DNA poorly or not at all (116). However, to evaluate the role of receptor editing in antidsDNA binding, one must compare the expressed H/L pairs to the "missing" H/L pairs, i.e. to those $\mathrm{H} / \mathrm{L}$ pairs that are the precursors of receptor editing and thus are excluded from the peripheral repertoire. To analyze the missing VH/VL pairs, we re-derived and expressed them as bivalent single-chain $\mathrm{Fv}(\mathrm{scFv})$ in E. coli. For comparison, we generated $\mathrm{VH} / \mathrm{VL}$ pairs that are expressed in anti-DNA $\mathrm{H}$ chain tg mice. The structure of the recombinant $\mathrm{VH} / \mathrm{VL}$ pairs provided a technical advantage over the IgM antibodies: 

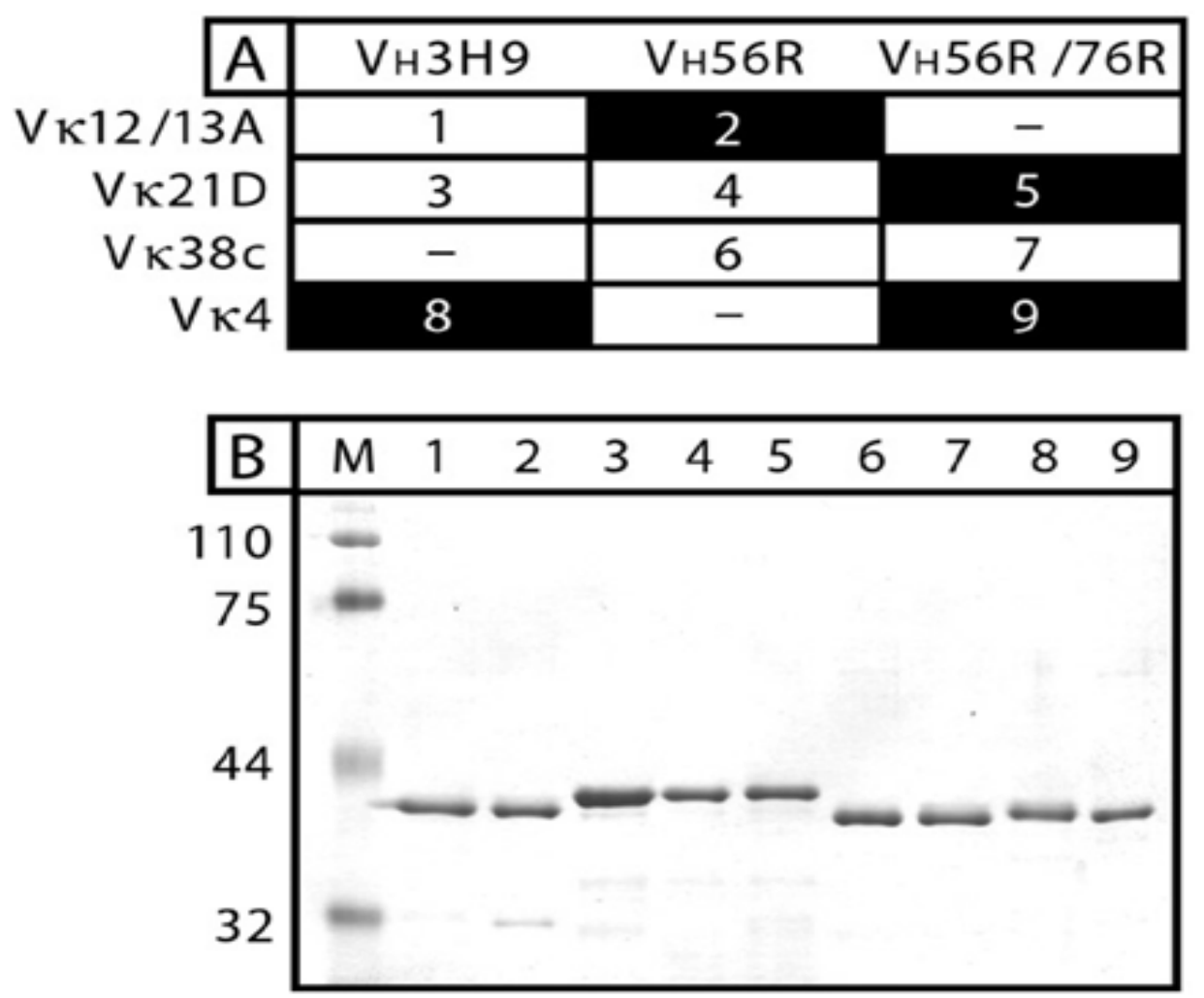

Figure 2.1 Construction of bivalent variable fragments.

Our system for bivalent scFv expression in E.coli (234) and the H chain variants of 3H9 (170) were previously described. We cloned the murine Vк12/13A (referred to as 12-46 by Thiebe et al.) (33) as a rearrangement to $\mathrm{J}_{\kappa} 2$ from the Balb/c hybridoma $7942-\mathrm{H} 40$ (235) by using the oligonucleotide primers Vк12/13 amino (5'-GCG TGT GGA TCC GAC ATC CAG ATG ACT CAG-3') and Jк2 (5'-GCG GTT AGA TCT CCA GCT TGG TCC CCC CT-3'). The primers introduced unique restriction sites at the amino and carboxy termini of the $\mathrm{V}_{\mathrm{L}}$ domain that were complementary to sites in our expression

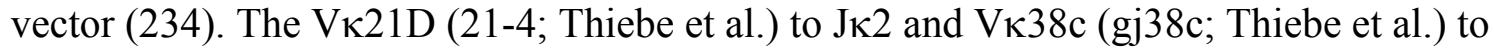
$\mathrm{J} \kappa 1$ rearrangements were similarly amplified from hybridoma DNA (116) by using primers Vк21D amino (5'-GCG GTT AGA TCT GAC ATT GTG CTG ACC CAA TCT3') and $\mathrm{J}_{\kappa} 2$ or $\mathrm{V \kappa} 38 \mathrm{c}$ amino (5'-GCG TGT GGA TCC GAC ATC CAG ATG ACA CAG-3') and Jк1 (5'-GCG GTT AGA TCT CCA GCT TGG TGC CTC CA-3') respectively. In this manner, nine $\mathrm{H} / \mathrm{L}$ chain combinations were constructed. Each $\mathrm{scFv}$ was fused in-frame to the c-jun leucine zipper to generate bivalent Fv that contained the domain B of Staphylococcal protein A for detection and a carboxy terminal His-tag for purification of the fusion protein (234). 


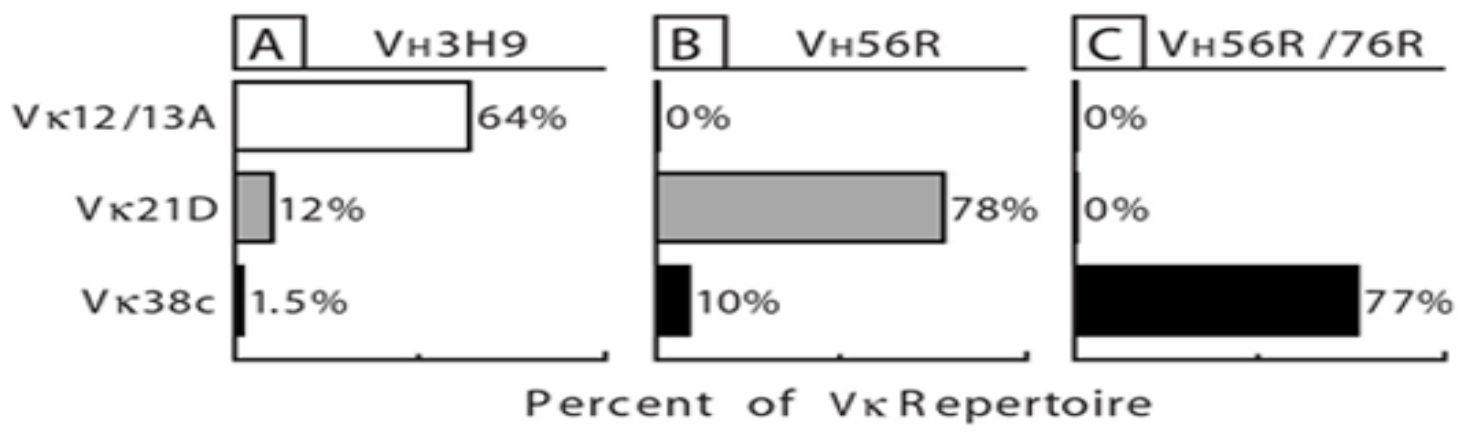

Figure 2.2 H chain Tg BALB/c mice show drastic differences in their L chain repertoires.

The endogenous L chain repertoires in anti-DNA $\mathrm{H}$ chain tg mice have previously been analyzed by constructing LPS hybridomas from splenic B cells $(116,122)$. Vк12/13A predominates in LPS-stimulated B cell hybridomas from VH3H9 mice $(116,122)$, with

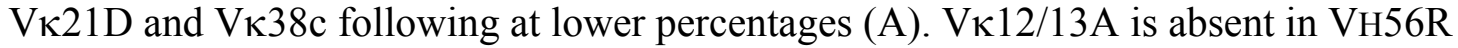
Tg mice (116), whereas $V \kappa 21 \mathrm{D}$ predominates and $\mathrm{V} \kappa 38 \mathrm{c}$ is less prevalent $(\mathrm{B}) . \mathrm{V} \kappa 38 \mathrm{c}$ is

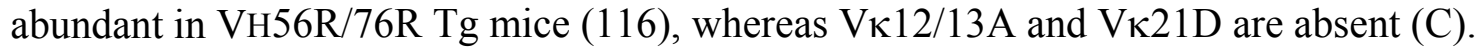
Additional $\mathrm{V} \kappa$ are expressed at low frequencies but not represented in the figure. 
The bivalent binding of our $\mathrm{scFv}$ made them useful substitutes for measuring BCR interactions with antigen. All together, we constructed nine scFv, five to represent $\mathrm{VH} / \mathrm{VL}$ pairs expressed in the spleen and four to represent pairs that are missing from the peripheral repertoire (Figure 2.1).

Our initial analysis focused on the most closely related VH/VL pairs: those in which a single residue in VH determines whether a VL participates in the splenic B cell repertoire or not (Figure 2.2). Thus, we compared VH3H9+Vк12/13, a pair that accounts for $64 \%$ of the repertoire in $\mathrm{VH} 3 \mathrm{H} 9$ mice (Figure 2.2A) to $\mathrm{VH} 56 \mathrm{R}+\mathrm{V} \kappa 12 / 13$, a pair that is absent from the repertoire in VH56R mice (Figure 2.2B), and VH56R+Vк21D, a pair that accounts for $78 \%$ of the repertoire in VH56R mice (Figure 2.2B) to $\mathrm{VH} 56 \mathrm{R} / 76 \mathrm{R}+\mathrm{V \kappa} 21 \mathrm{D}$, a pair that is absent from the repertoire of VH56R/76R mice (Figure 2.2C). The absence of Vk12/13 from the VH56R repertoire results from the single replacement of Asp in VH3H9 by Arg at position 56 in VH56R. Similarly, the absence of VK21D from the VH56R/76R repertoire is a result of introducing a second Arg (at position 76) into the VH56R/76R tg. Because these Args increase the affinity of the VH for dsDNA (115), we predicted that the missing pairs would provide an insight into how increased dsDNA binding limits the number of acceptable editor $\mathrm{L}$ chains.

Indeed, these scFvs displayed a gradient of binding to dsDNA with $\mathrm{VH} / \mathrm{VL}$ pairs that are expressed in the spleen having little or no dsDNA binding and pairs that are absent from the repertoire exhibiting avid binding (Figure 2.3A). Hence, the binding

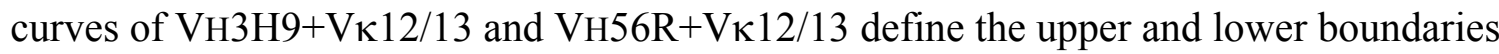
of the interval of relative anti-dsDNA affinities that contains the threshold between affinities that allow B cell maturation and affinities that prevent it (Figure 2.3A).

\subsubsection{DNA and PS binding of Vк38c combinations}

The Vк38c contributes to the B cell repertoire in VH56R mice (Figure 2.2B) and it is the predominant $\mathrm{V} \kappa$ in $\mathrm{VH} 56 \mathrm{R} / 76 \mathrm{R}$ mice (Figure 2.2C). Therefore, we expected that $\mathrm{V} \kappa 38 \mathrm{c}$ would have characteristics of $\mathrm{L}$ chains that edit anti-dsDNA specificity, i.e. reduced avidity to dsDNA. In previous experiments with hybridoma-derived $\operatorname{IgM}(116$,

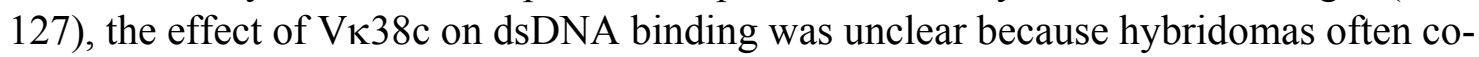
expressed V $\lambda 1$, a L chain that is an effective dsDNA binder (115).

The present experiments established that pairs between Vк38c and either VH56R or VH56R/76R do indeed bind DNA (Figure 2.3C). In addition, both $\mathrm{VH} / \mathrm{VL}$ pairs bound

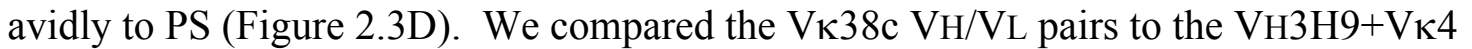
pair used by the $3 \mathrm{H} 9$ autoantibody, an anti-dsDNA that is excluded from the peripheral

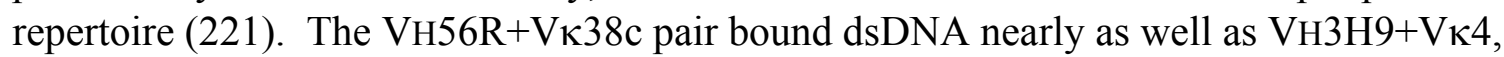
whereas VH56R/76R+Vk38c bound four-fold better (Figure 2.3C). To account for the presence of avid anti-dsDNA receptors in the splenic B cell repertoire, we considered that additional autospecificities could affect expression of these BCR. Consistent with this 

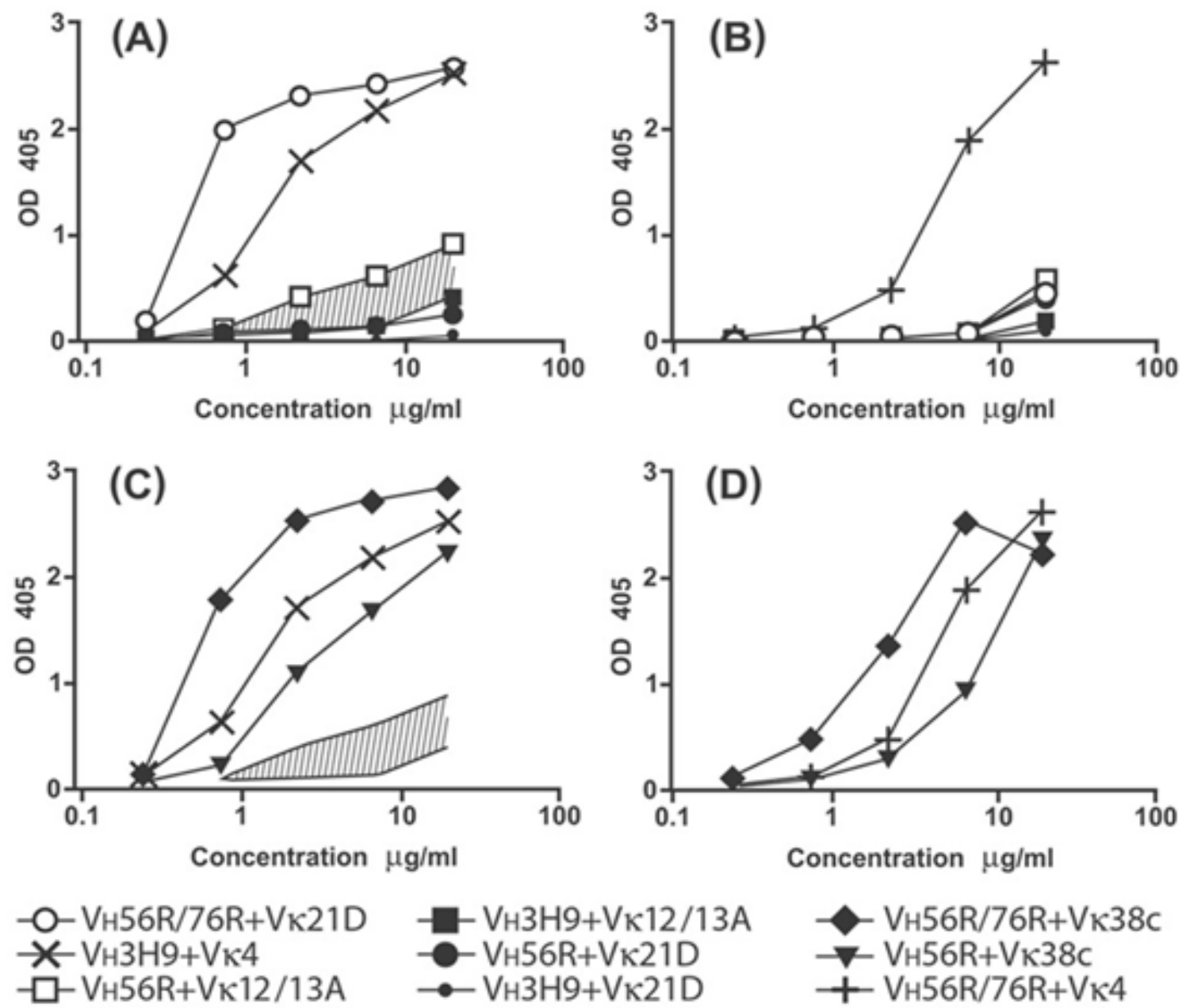

Figure 2.3 Binding of bivalent scFv to dsDNA and PS in ELISA.

At the given concentrations of scFv, absorbance was measured at $405 \mathrm{~nm}$. VH3H9+Vк4 and $\mathrm{VH56R/76R+V \kappa 4} \mathrm{were} \mathrm{used} \mathrm{as} \mathrm{positive} \mathrm{controls} \mathrm{for} \mathrm{dsDNA} \mathrm{(A,} \mathrm{C)} \mathrm{and} \mathrm{PS} \mathrm{binding}$ $(B, D)$, respectively. The area shaded in (A) and (C) indicates a range of relative antiDNA affinities that includes a proposed threshold of DNA binding responsible for inducing receptor editing. The $\mathrm{H} / \mathrm{L}$ pairs that are represented in the repertoire fall below the threshold, those that are absent bind DNA above the threshold. H/L pairs that incorporate $\mathrm{V} \kappa 38 \mathrm{c}$ exceed the proposed threshold and react strongly with dsDNA (C) and PS (D). 
idea, previous work on anti-dsDNA autoantibodies found that they often react with other autoantigens, such as chromatin, ribonucleoproteins, or membrane lipids (172).

\subsubsection{Specificity of VH/VL pairs for intracellular antigens}

To test whether the anti-dsDNA VH/VL have an additional autospecificity, we used Jurkat $\mathrm{T}$ cells as a source of diverse antigens. Indeed, immunohistochemistry

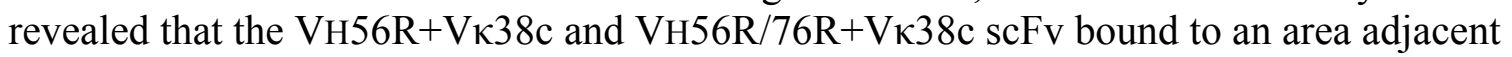
to the nucleus (Figure 2.4A and 2.4B), whereas VH3H9+Vk21D did not show intracellular binding (Figure $2.4 \mathrm{G}$ ), and $\mathrm{VH} 56 \mathrm{R} / 76 \mathrm{R}+\mathrm{V \kappa} 4$ bound preferentially to nuclear antigens (Figure $2.4 \mathrm{H}$ ).

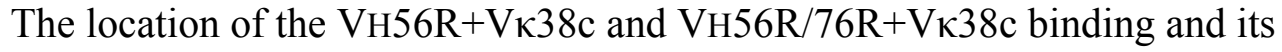
distribution in the form of lamellar stacks and tubular bundles suggested binding to stacks and projections of the Golgi. The scFv binding paralleled the binding of Griffonia simplicifolia lectin II, a lectin that binds terminal GlcNAc sugars enriched in the Golgi

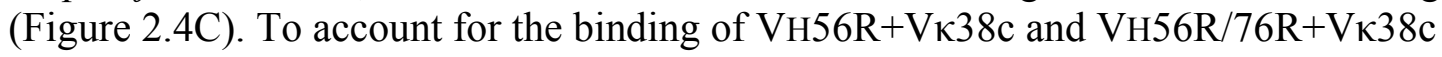
$\mathrm{scFv}$ to the Golgi, we considered whether the scFv and the lectin bind to terminal GlcNAc moieties. However, we observed only limited colocalization between the $\mathrm{VH} 56 \mathrm{R}+\mathrm{V \kappa} 38 \mathrm{c} \mathrm{scFv}$ and lectin II, in spite of the fact that both bound in the immediate vicinity of each other (Figure 2.4D-F). Because the binding of the scFv was more limited and diffuse in the presence of the lectin (Figure 2.4D-F) than in its absence (Figure 2.4A), it is likely that the lectin sterically hinders access of the scFv to its antigen.

\subsubsection{Distribution of Ig in hybridomas}

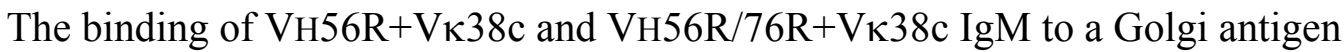
may alter the intracellular distribution of $\operatorname{IgM}$ in situ. To assess this possibility, we examined hybridomas expressing Vк38c from VH56R and VH56R/76R tg C57BL/6 mice

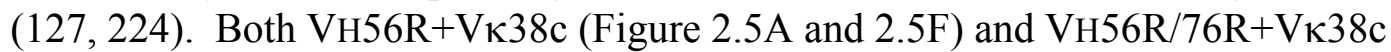
(Figures $2.5 \mathrm{C}$ and $2.5 \mathrm{D}$ ) IgM formed a dense aggregate in the interior of the hybridoma cells. In contrast, the IgMs with other editors, VH56R/Vк21D (Figure $2.5 \mathrm{~B}$ and $2.5 \mathrm{G}$ ) or VH56R/V $\lambda x$ (Figure 2.5E), were distributed at or just beneath the cell surface, leaving the center of the cell free of Ab. Therefore, the specificity of the tg VH and Vк38c pairs leads to the accumulation of the IgM in the interior of the cells and may reflect binding between the newly synthesized IgM and an antigen that is expressed in the Golgi.

Because $\operatorname{IgM}$ and IgG isotypes differ in their assembly requirements, we tested whether constant region isotype affects intracellular retention. We took advantage of the fact that B cells expressing VH56R/76R+Vк38c switch to IgG in MRL-lpr/lpr mice (232). IgG hybridomas derived from autoimmune VH56R/76R mice express

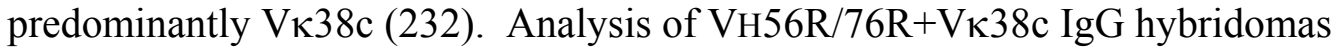
demonstrated that class switch did not change the intracellular accumulation of the $\operatorname{IgG}$ 
Figure 2.4 Analysis of scFv binding to Jurkat cells.

scFv binding to fixed and permeabilized cells was visualized with AF647 rabbit antimouse IgG (displayed in red) and nuclei with Sytox Orange (blue). The VH56R+Vк38c (A) and $\mathrm{VH} 56 \mathrm{R} / 76 \mathrm{R}+\mathrm{V \kappa} 38 \mathrm{c} \mathrm{scFv}(\mathrm{B})$ bind to a perinuclear domain composed of tubular bundles and lamellar stacks that likely corresponds to the Golgi apparatus. Binding of AF488 lectin II (green) identifies terminal GlcNAc moieties that are enriched in the Golgi (C). The location of $\mathrm{VH} 56 \mathrm{R}+\mathrm{V} \kappa 38 \mathrm{c} \mathrm{scFv}$ binding is surrounded by lectin II-

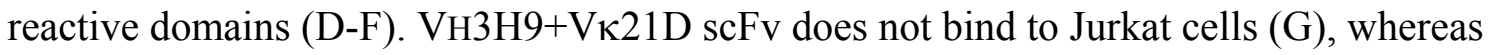
$\mathrm{VH} 56 \mathrm{R} 76 \mathrm{R}+\mathrm{V} \kappa 4 \mathrm{scFv}$ binds predominantly to the nucleus $(\mathrm{H})$. Image of lectin II binding (I). Separate RGB channels are shown at one-third size below the composite color images for panels D through I. Binding of scFv to Jurkat cells was indistinguishable from the binding to mouse thymocytes. 


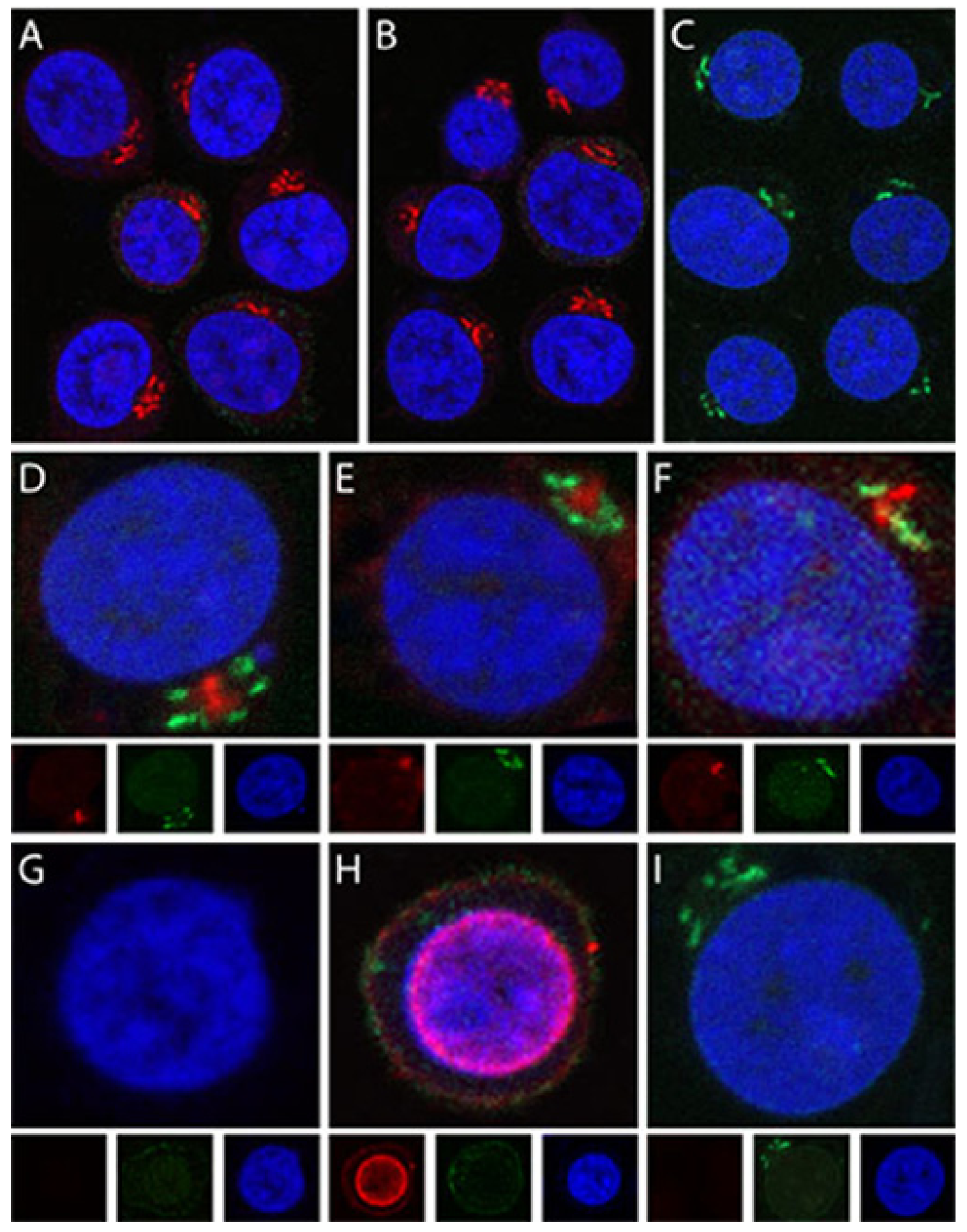


Figure 2.5 Distribution of IgM in B cell hybridomas and primary B cells.

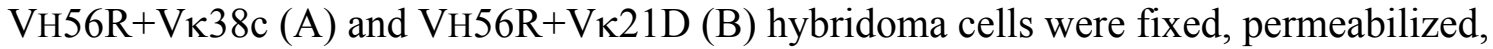
and incubated with AF467 goat anti-mouse IgM (red), AF488 lectin II (green), and Sytox Orange (blue). The VH56R+Vк38c IgM accumulate inside the cell (A). Cultures of $\mathrm{VH} 56 \mathrm{R}+\mathrm{V \kappa} 38 \mathrm{c}$ contain cells with fragmented nuclei and apoptotic phenotype [Insert]. In contrast, $\mathrm{VH} 56 \mathrm{R}+\mathrm{V \kappa} 21 \mathrm{D}$ IgM are localized on or close to the cell surface and little or no

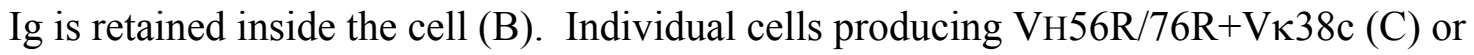
$\mathrm{VH} 56 \mathrm{R}+\mathrm{V} \kappa 38 \mathrm{c} \operatorname{IgM}(\mathrm{F})$. IgM cluster at the cell surface (Panels A \& C, arrowheads).

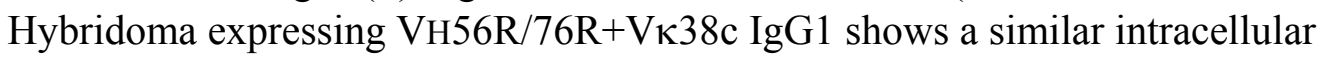
accumulation of the Ig but smaller clusters at the plasma membrane (D). Individual $\mathrm{VH} 56 \mathrm{R}+\mathrm{V} \lambda \mathrm{x}(\mathrm{E})$ and $\mathrm{VH} 56 \mathrm{R}+\mathrm{V} \kappa 21 \mathrm{D}(\mathrm{G})$ hybridoma cells show IgM receptors that are distributed at or near the cell surface. Cells that have lost expression of IgM do not bind

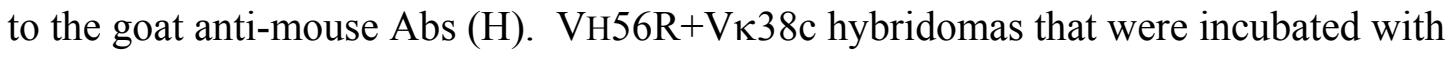
anti-mouse IgM, then fixed and stained with Sytox orange, show cell surface clusters of $\operatorname{IgM}(\mathrm{I})$. VH56R+Vк21D hybridomas, when treated the same, show reduced levels of surface IgM (J). B cells from the spleen of a VH56R mouse that were sorted for low surface IgM ${ }^{\mathrm{a}}$, then grown in culture for $48 \mathrm{~h}$, fixed, and stained as in (A) above, show cytoplasmic IgM aggregates (K). Discrete clusters of IgM appear at the cell surface. The intracellular IgM distribution in these primary B cells is strikingly similar to the IgM

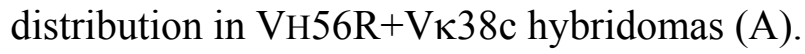



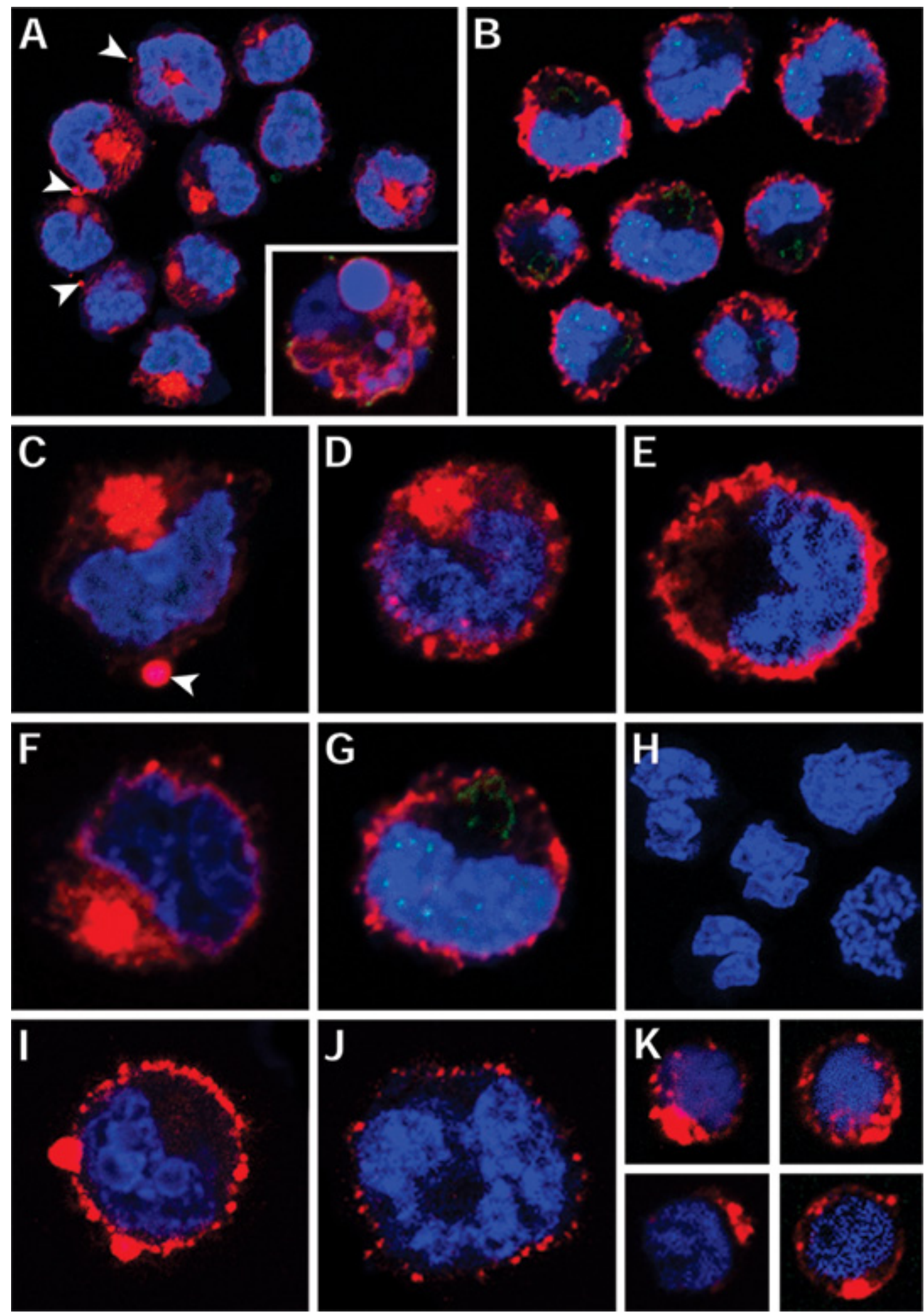
(Figure 2.5D), although the IgG aggregates were smaller and more widely distributed throughout the cytoplasm than the IgM aggregates.

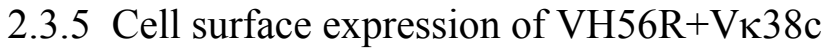

Given the intracellular accumulation of $\mathrm{VH} 56 \mathrm{R}+\mathrm{V \kappa} 38 \mathrm{c}$, we wondered whether

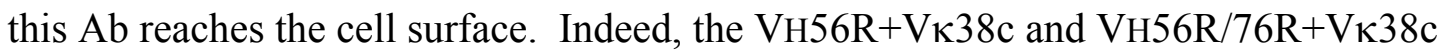
IgM, rather than being degraded, appeared in clusters at the plasma membrane (Fig 2.5A and $2.5 \mathrm{C}$, arrow heads). To test whether this IgM is also exposed at the cell surface, we stained live hybridomas (see methods) and observed that the VH56R+VК38c IgM forms large surface IgM clusters (Figure 2.5I). Such large surface IgM clusters were not observed on more than 200 cells expressing VH56R+Vк21D. Moreover, the surface density of $\mathrm{VH} 56 \mathrm{R}+\mathrm{V} \kappa 38 \mathrm{c} \operatorname{IgM}$ is higher than $\mathrm{VH} 56 \mathrm{R}+\mathrm{V} \kappa 21 \mathrm{D}$. Thus, even though $\mathrm{VH} 56 \mathrm{R}+\mathrm{V \kappa} 38 \mathrm{c}$ and $\mathrm{VH} 56 \mathrm{R} / 76 \mathrm{R}+\mathrm{V} \kappa 38 \mathrm{c}$ IgM accumulate in the cytoplasm, these receptors also translocate to the cell surface.

Even though VH56R+Vк38c hybridomas accumulate intracellular IgM and express IgM at the cell surface, the distribution of IgM in vivo might be different. If Golgi binding blocks or delays antibody transport to the cell surface, then surface levels of $\mathrm{VH} 56 \mathrm{R}+\mathrm{V \kappa} 38 \mathrm{c}$ IgM may be reduced compared to other $\mathrm{VH} / \mathrm{V} \kappa$ pairs. To determine surface IgM levels in newly forming B cells, we analyzed B220+, AA4.1+ cells (Hardy fraction $\mathrm{E}$ ) in the BM of VH56R Tg C57BL/6 mice. We used $\kappa$ staining to compare surface IgM levels in B cells expressing the $\operatorname{IgM}^{\mathrm{a}}$ allotype (that should include $\mathrm{VH} 56 \mathrm{R}+\mathrm{V} \kappa 38 \mathrm{c}$-expressing B cells) to B cells expressing the $\operatorname{IgM}^{\mathrm{b}}$ allotype. In two independent experiments (one of which is shown in Figure 2.6), we observed reduced $\kappa$ surface staining in $\operatorname{IgM}^{\mathrm{a}}$ than in $\operatorname{IgM}^{\mathrm{b}}$ cells. A lower level of $\kappa$ expression was also observed in $\operatorname{IgM}^{\mathrm{a}+}$ recirculating BM B cells (Hardy Fraction F), splenic transitional cells, and mature B cells from VH56R Tg C57BL/6 mice (Figure 2.6). These findings are consistent with the model that binding to an intracellular antigen impairs antibody trafficking to the cell surface.

To examine the intracellular distribution of $\operatorname{IgM}$ in primary B lymphocytes, we isolated B cells from the spleen of a VH56R mouse and maintained them in short-term culture prior to confocal microscopy. We sorted for $\operatorname{IgM}^{\mathrm{a}}$-positive B cells, in order to exclude cells expressing endogenous $\mathrm{VH}$ from our analysis (Figure $2.5 \mathrm{~K}$ ).

Approximately one third (35\%) of B cells exhibited intracellular IgM, the distribution

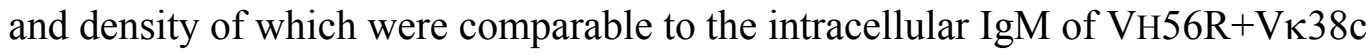
hybridomas. In addition, some of the IgM receptors formed clusters at the cell surface (Figure $2.5 \mathrm{~K}$ ).

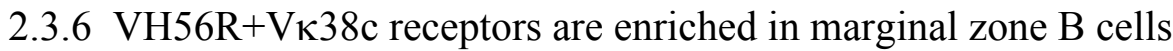

To analyze the expression of $\mathrm{VH} 56 \mathrm{R}+\mathrm{V \kappa} 38 \mathrm{c} \mathrm{BCR}$ in B cell subsets, splenocytes were sorted as transitional (Tr) $\left(\mathrm{B} 220^{+}, \mathrm{AA} 4.1^{+}\right)$, follicular $(\mathrm{Fo})\left(\mathrm{IgMa}{ }^{\mathrm{int}}, \mathrm{CD} 21^{\mathrm{int}}\right)$, and 
Figure 2.6 Surface $\kappa$ staining intensity in $\operatorname{IgM}^{\mathrm{a}}$ versus $\operatorname{IgM}^{\mathrm{b}}$ gated $\mathrm{B}$ cell populations from VH56R Tg C57BL/6 mice.

Single cell suspensions were prepared from BM and spleens of 3-6 month old B6.56R mice $(n=2$ mice for $B M$ analysis and 3 mice for spleen analysis in three separate experiments, representative plots are shown). Cell suspensions were stained with antibodies to B220-Alexa 750, IgM ${ }^{\mathrm{a}}$-FITC, IgM ${ }^{\mathrm{b}}$-PE, CD21-APC, AA4.1-PE-Cy7 and kappa- biotin, revealed with SA-PerCP-Cy5.5 as described in Methods. Dead cells were excluded by DAPI staining, lymphocytes were gated on the basis of forward vs. side scatter and singlets on the basis of pulse width. Fluorescence data on one million events per tube for BM and 50,000 events for spleen were acquired on an FACSAria cell sorter (BD Biosciences) and analyzed. $\operatorname{IgM}^{\mathrm{a}+}$ (red line) and $\operatorname{IgM}^{\mathrm{b}+}$ (blue line) cells in each of the gated populations defined in the figure were analyzed separately for $\kappa$ staining intensity. Kappa staining intensity is plotted as a function of the number of events in panels A-E. 
A. Hardy fraction E (B220+, AA4.1+)

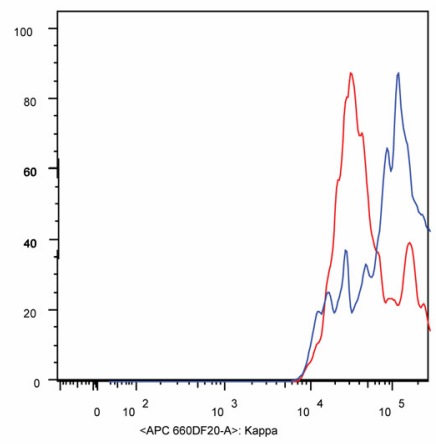

B. Hardy fraction F (B220+, AA4.1-)

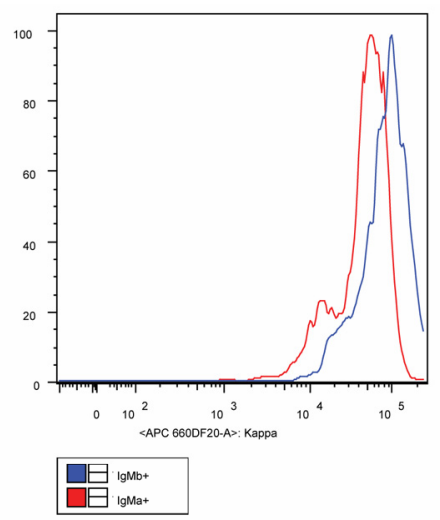

C. Spl transitional cells (B220+, AA4.1+)

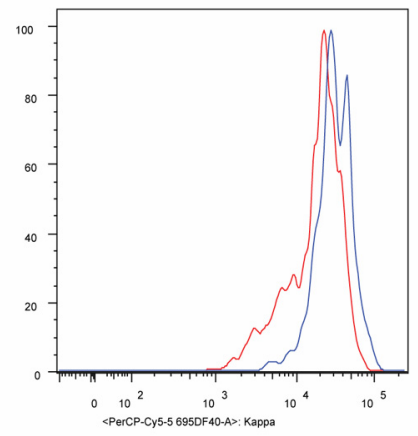

D. Follicular cells (B220+, CD21int)

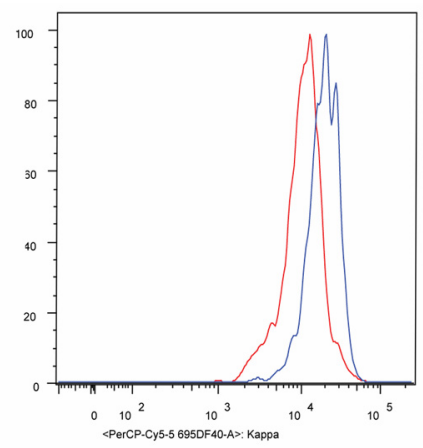

E. Marginal zone cells (B220+, CD21++)

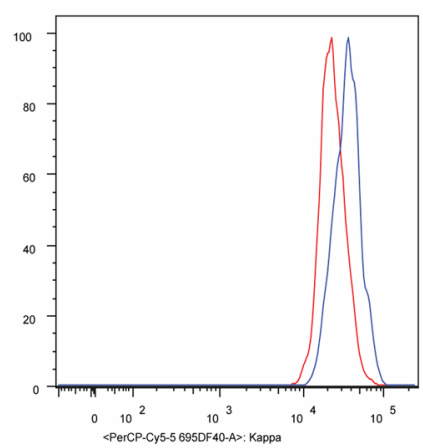


$\mathrm{MZ}\left(\mathrm{IgMa}{ }^{\text {hi }}, \mathrm{CD} 21^{\text {hi }}\right.$ ) B cells (Figure 2.7A and B). Rearrangements of $\mathrm{V} \kappa 38 \mathrm{c}$ to $\mathrm{J} \kappa 5$, the main products of editing by $V \kappa 38 \mathrm{c}$, were measured in each of the subsets by quantitative PCR of genomic DNA. The results are depicted as fold differences relative to $\mathrm{IgM}^{+} / \mathrm{\kappa}^{+}$cells from wild type $\mathrm{C} 57 \mathrm{BL} / 6$ mice (Figure $2.7 \mathrm{C}$ ). In $\mathrm{Tr}$ cells, there was a two-fold increase over wild-type mice in the use of $\mathrm{V \kappa} 38 \mathrm{c}$. However, compared to wild type B cells, Vк38c was enriched approximately six-fold in Fo B cells, and nearly

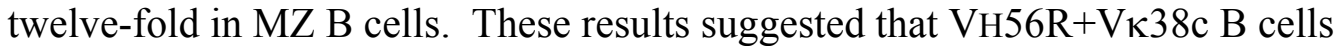
preferentially acquire a MZ phenotype and accumulate in the splenic MZ. Because the $\mathrm{MZ}$ is expanded in VH56R mice, in absolute numbers, there are nearly twice as many $\mathrm{MZ}$ than Fo B cells that express VH56R+Vк38c.

Using surface IgM density as a basis for assigning B cells to the MZ subset could be problematic in the case of $\operatorname{IgM}$ with unusual distribution or trafficking in B cells. To provide an independent measure of $\mathrm{VH} 56 \mathrm{R}+\mathrm{V} \kappa 38 \mathrm{c}$ use in the major B cell subsets, and to examine VH56R and Vк38c use in individual cells, we separated B cells based on CD21 and CD23 expression (Figure 2.7D-G), and analyzed their VH and VL use by single cell $\mathrm{V}$ gene RT-PCR. We observed that $35 \%$ of MZ B cells expressed both VH56R and

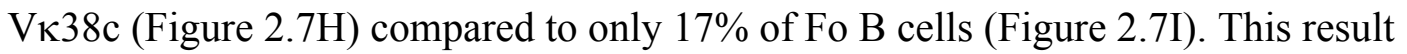
confirmed the data from the quantitative PCR analyses and established that the MZ contains the highest accumulation of the autoreactive $\mathrm{VH} 56 \mathrm{R}+\mathrm{V} \kappa 38 \mathrm{c}$ pair in the spleen. Moreover, these results indicated that $\mathrm{V} \kappa 38 \mathrm{c}$ and $\mathrm{VH} 56 \mathrm{R}$ are co-expressed in individual $\mathrm{MZ}$ B cells, a result that could not be derived from quantitative PCR with B cell populations.

\subsection{Discussion}

B cells become tolerant to self-antigens by replacing autoreactive L chains with $\mathrm{L}$ chains that suppress or modify the original autoreactivity. Several L chains edit anti-

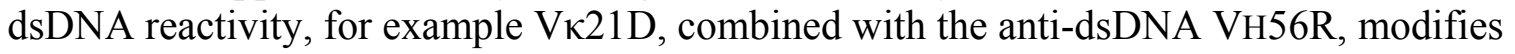
the combining site (116) so as to curtail dsDNA binding (Figure 2.3A). Other L chains such as $\mathrm{V} \kappa 8$ decrease affinity for dsDNA, and $\mathrm{VH} 3 \mathrm{H} 9+\mathrm{V} \kappa 8 \mathrm{~B}$ cells are in the periphery in a quasi-anergic state(221). Also in the periphery is the combination of $\mathrm{VH} 56 \mathrm{R}+\mathrm{V \kappa} 38 \mathrm{c}$, even though Vк38c does not decrease dsDNA binding (Figure 2.3C). Our data suggest a possible reason for the escape of this $\mathrm{VH} / \mathrm{VL}$ pair from central tolerance: the $\mathrm{VH} 56 \mathrm{R} / \mathrm{V \kappa} 38 \mathrm{c}$ has an additional specificity for a Golgi-associated antigen (Figure 2.4A). Because newly synthesized IgM must transit through the Golgi on its way to the cell surface, the binding of VH56R+Vк38c IgM to the Golgi may delay surface IgM expression. Consistent with this idea, surface $\kappa$ staining is less intense in $\operatorname{IgM}^{\mathrm{a}}$ than in $\mathrm{IgM}^{\mathrm{b}} \mathrm{B}$ cells (Figure 2.6). It is possible that the $\mathrm{VH} 56 \mathrm{R}+\mathrm{V \kappa} 38 \mathrm{c} \mathrm{B}$ cells might have not been taken into consideration for this analysis but clearly, alternative explanations for the reduced levels of surface Ig in $\operatorname{IgM}^{\mathrm{a}} \mathrm{B}$ cells may apply, for example L chain editing and/or surface Ig down-regulation $(131,220,230,236)$.

It is well established that intracellular Ig signals can promote B cell development. In preB cells, the preB receptor acts from an intracellular location to generate most, if not 
Figure $2.7 \mathrm{V \kappa} 38 \mathrm{c}-\mathrm{J} \kappa 5 \mathrm{~L}$ chain rearrangements in peripheral B lymphocyte subsets.

Lymphocytes from the spleen of a VH56R/B6 mouse were separated into Tr and mature $\mathrm{B}$ cell fractions based upon B220 and AA4.1 expression (A). B220 ${ }^{+}$splenocytes were further separated into MZ and Fo B cell subsets. Quantitative PCR was used to

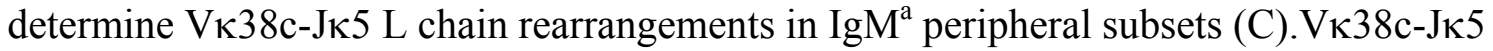
rearrangements are depicted relative to a C57BL/6 wild type mouse. Significance levels were determined by two-tailed Student's t-test: *, $\mathrm{p} \leq 0.01$. Alternatively, B220 lymphocytes (D) were sorted into MZ (CD2 $\left.1{ }^{\text {hi }} \mathrm{CD} 23^{\text {int }}\right)$ and Fo B cells $\left(\mathrm{CD} 21^{\text {int }} \mathrm{CD} 23^{\text {hi }}\right)$, as previously (127) reported (E). Purity of sorted MZ and Fo B cells was $98.2 \%$ and 98.8\% respectively (F and G). Single cell RT-PCR identified VH56R and Vк38c

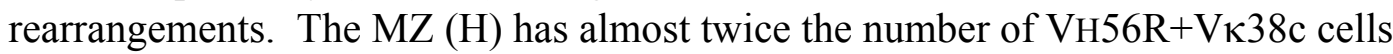
(35.2\%) compared to the Fo (16.8\%; I). About 34\% of the cells in the MZ have VH56R and a $\mathrm{L}$ chain other than $\mathrm{V} \kappa 38 \mathrm{c}$, whereas $\mathrm{V} \kappa 38 \mathrm{c}$ is associated with an endogenous $\mathrm{H}$ chain in $14 \%$ of the cells $(H)$. In $9.5 \%$ of the Fo B cells, Vк38c is associated with an endogenous $\mathrm{H}$ chain (I). 

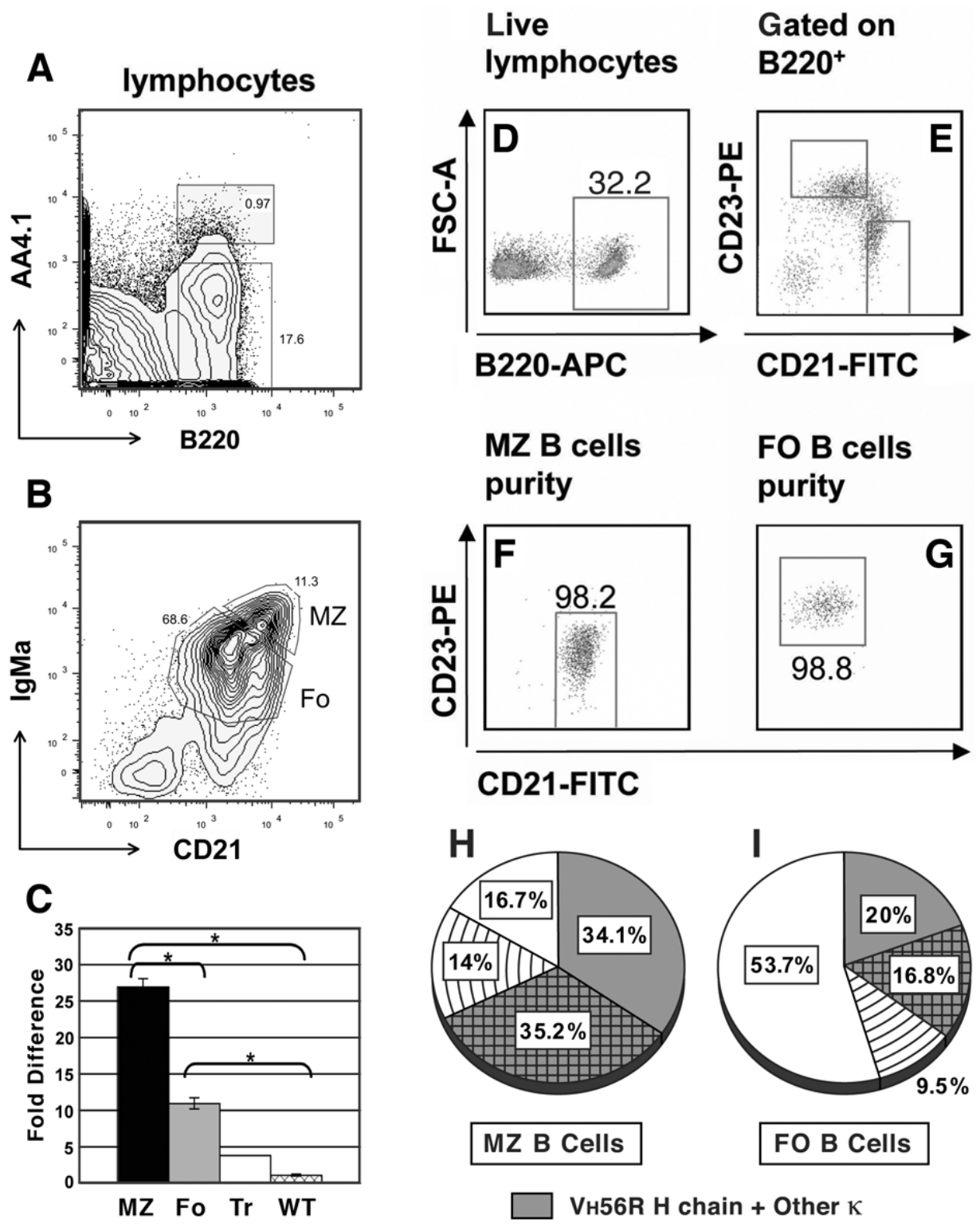

$$
\begin{aligned}
& \begin{array}{|l|l|}
\hline \text { MZ B Cells } & \text { FO Cells } \\
\hline
\end{array} \\
& \text { VH56R H chain + Other } \kappa \\
& \text { 囲田 Vн56R + VК38c } \\
& \text { E Other } \mathrm{H} \text { chain }+\mathrm{V}_{\kappa} \mathbf{3 8 c} \\
& \square \text { Other } \mathrm{H} \text { chain + Other } \mathrm{K}
\end{aligned}
$$


all, signals that promote B cell differentiation (237). Similarly, intracellular IgM may be competent for signaling because the BCR assembles and associates with $\operatorname{Ig} \alpha$ and $\operatorname{Ig} \beta$ in a post-ER compartment (238). Furthermore, there is precedent for the development of peripheral B cells whose BCR binds to an intracellular antigen: B cells that express IgM to hen egg lysozyme (HEL), together with an ER-anchored form of the antigen, block surface IgM expression by Ig retention in the ER, yet they develop into splenic Fo B cells (239).

Unlike the anti-HEL-ER B cells, the VH56R+Vк38c B cells accumulate IgM in the Golgi but they nevertheless achieve high surface IgM density (Figure 2.8). In addition, they also acquire a MZ B cell phenotype (Figure 2.7). The different outcomes in the anti-HEL-ER model and the anti-dsDNA-Golgi model may reflect differences in the sites of IgM accumulation. IgMs such as the anti-HEL-ER that accumulate in the ER may be diverted to aggresomes, sites of increased protein degradation (240), whereas the $\mathrm{VH} 56 \mathrm{R}+\mathrm{V \kappa} 38 \mathrm{c}$ IgM that accumulates in the Golgi may not be degraded. Consequently, accumulation of $\mathrm{VH} 56 \mathrm{R}+\mathrm{V \kappa} 38 \mathrm{c}$ IgM might induce the unfolded protein response, an adaptation known to play a role in the B cell response to antigen (241). The unfolded protein response may drive the $\mathrm{VH} 56 \mathrm{R}+\mathrm{V \kappa} 38 \mathrm{c}$ B cells to become surface IgM-bright because it increases the efficiency of IgM synthesis. The increased IgM synthesis may reach a level that exceeds the intracellular retention capacity of the Golgi. As result, large IgM clusters may be transfered to the cell surface (Figure 2.5I).

The surface expression of the autoreactive VH56R+Vk38c IgM may be responsible for the increased abundance of VH56R+Vк38c B cells in the MZ (Figure 2.7). It has been argued that sustained signaling through the $B C R$ promotes $B$ cell homing to the splenic MZ (160). B cells sequestered in the MZ may play an important role in peripheral tolerance (242). Because MZ B cells usually lack help to undergo affinity maturation or switch isotype, they may be blocked in the secretion of pathogenic antibodies. This possibility could account for the fact that B cells expressing $\mathrm{VH} 56 \mathrm{R}+\mathrm{V \kappa} 38 \mathrm{c}$ receptors, despite being autoreactive and achieving high surface Ig density, do not spontaneously induce autoimmunity. 

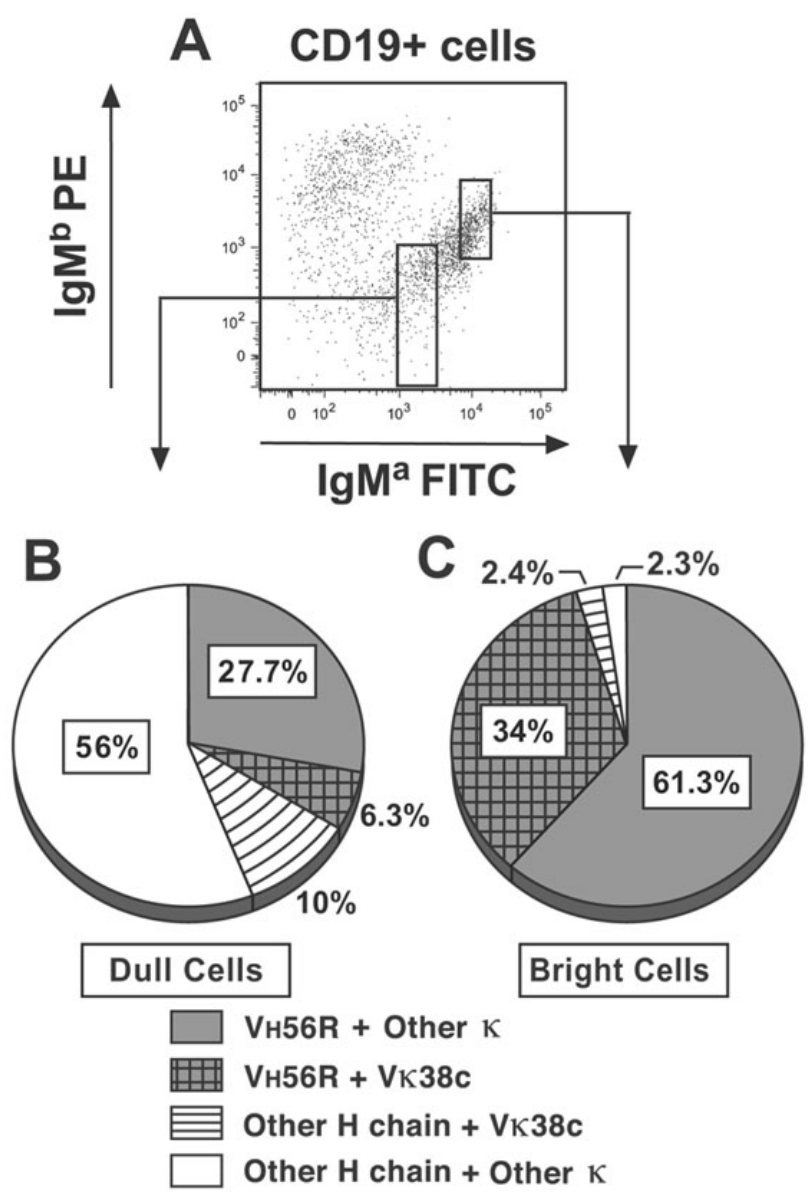

Figure 2.8 VH56R and Vא38c secondary rearrangements are more abundant in surface IgM ${ }^{\mathrm{a}}$-bright $\mathrm{B}$ cells.

$\mathrm{CD} 19^{+}$lymphocytes isolated from the spleen of $56 \mathrm{R} / \mathrm{Bb}^{+/-} \mathrm{Tg}$ mouse were sorted into $\operatorname{IgM}^{\text {a }}$-bright and IgM ${ }^{\text {a }}$-dim cells (A). Nested Single cell PCR revealed that $6.3 \%$ of the $\mathrm{IgM}^{\mathrm{a}}$-dim cells have $\mathrm{VH} 56 \mathrm{R}+\mathrm{V} \kappa 38 \mathrm{c}$ rearrangements while $27.2 \%$ have $\mathrm{VH} 56 \mathrm{R}$

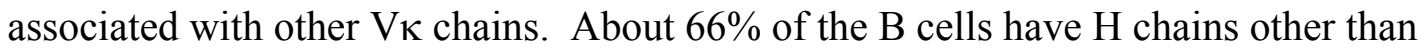
VH56R and $10 \%$ of such cells express Vк38c (B). Among the surface IgM ${ }^{\mathrm{a}}$-bright cells, $34 \%$ have the VH56R and VK38c rearrangements, whereas $61.3 \%$ have retained the 56R $\mathrm{H}$ chain but are associated with other $\mathrm{V \kappa}$. A small percentage of B cells (2.4\%) have $\mathrm{V \kappa} 38 \mathrm{c}$ that pairs with a $\mathrm{H}$ chain other than 56R and about $2.3 \%$ of the cells have neither VH56R nor Vк38c (B). 


\section{CHAPTER 3: EXTRUSION OF IgM POLYMER AGGREGATES "SPHERONS" AND SECRETION OF IgM HEMIMERS FROM B CELL HYBRIDOMAS THAT ACCUMULATE IgM IN THE GOLGI}

\subsection{Introduction}

A secreted IgM molecule is composed of 21 polypeptide chains when it contains a $\mathrm{J}$ chain as part of an IgM pentamer, or 24 chains when it is a hexamer of the basic $\mathrm{H}_{2} \mathrm{~L}_{2}$ monomer structure (243-245). Initiation of the assembly of secreted IgM occurs in the lumen of the ER and assembly requires accurate timing of backbone folding, efficient exchange of chaperones for permanent $\mathrm{H} / \mathrm{L}$ partners, the formation of inter- and intramolecular stabilizing bonds (246), and stage-specific addition of carbohydrates (247). The $\mu \mathrm{Hc}$ co-translationally associates with the Hc binding protein (BiP). BiP dissociates when the Lc pairs with the Hc $(248,249)$. The $\mu_{2} \mathrm{~L}_{2}$ monomers serve as the basic subunit for IgM polymerization (250). Not surprisingly, such a complex assembly process can be disrupted by numerous structural deficiencies.

Inappropriately folded or assembled IgM are retained in the ER and are subjected to proteasome-mediated degradation in the cytoplasm by a process called ER associated degradation (ERAD). ERAD maintains homeostasis in the ER and presumably this quality control mechanism ensures that beyond the ER, IgM are fully functional and correctly assembled $(251,252)$. If the synthesis of incorrectly assembled IgM exceeds the ability of proteasomes to degrade the IgM, intracellular aggregates of IgM form in the ER lumen that are referred to as Russell bodies $(106,253)$. The IgM also accumulates in the ER if proteasomal inhibitors are used due to rapid induction of pro-apoptotic UPR genes (254). On the other hand, misfolded proteins that exceed proteosomal degradation capacity are retro-translocated from the ER, to the cytosol where they aggregate as 'aggresomes' (255). In any case, a clearly identifiable defect in folding or assembly leads to protein accumulation in the ER or the cytoplasm.

While trying to understand the regulation of autoantibodies in non-autoimmune mice, we discovered an unusual type of anti-DNA B cell. This anti-DNA matures and joins splenic B cell subsets even though most other anti-DNA B cells are regulated at an immature stage of development (116). The fact that regulation of anti-DNA B cells coincides with the earliest expression of the BCR at the cell surface indicates that antigen engagement by BCR on immature B cells induces a block in B cell development. This block can only be relieved by revision/editing of the BCR or by functional inactivation of the $\mathrm{B}$ cell. This conclusion is consistent with studies on B cells with diverse antigen specificities.

The unusual anti-DNA BCR, when expressed in vitro, binds, in addition to DNA, a Golgi-associated antigen. The B cells expressing this BCR express lower levels of surface Ig at all stages of B cell development, thus suggesting a possible mechanism for their escape from regulation (256). If regulation of immature anti-DNA B cells depends on BCR engagement, then the reduced surface Ig expression may allow these B cells to 
escape central tolerance and participate in more mature B cell functions. These B cells reach the periphery and we can immortalize them as B cell hybridomas.

Here, we examine the intracellular IgM distribution in these anti-DNA B cell hybridomas. The hybridomas, expressing IgM composed of the VH56R Hc or VH56R/76R Hc and Vк38c Lc, in contrast to hybridomas expressing the same VH combined with other Lc, accumulate IgM in the medial Golgi, and secrete few if any soluble polymeric IgM. Instead, they extrude aggregates of polymeric IgM that we refer to as 'spherons' because of their shape. In addition, the hybridomas secrete monomeric $\operatorname{IgM}$.

\subsection{Methods}

\subsubsection{Animals}

The VH56R Tg has been described previously (116). VH56R mice were backcrossed on the C57BL/6 background for ten generations.

\subsubsection{Cell culture}

B cell hybridomas expressing VH56R+Vк38c IgM (H160.3) (127),

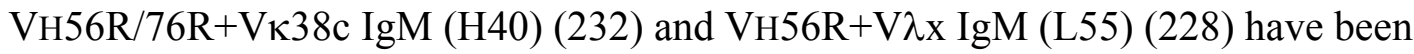
previously described (256). The 38C-13 lymphoma was a kind gift from Professor J. Haimovich, Tel Aviv University, Tel Aviv, Israel and WEHI-231 was a kind gift from Dr. A.K. Yi, University of Tennessee, Memphis, USA.

\subsubsection{Immunofluorescence and confocal microscopy}

Cells grown of glass cover slips were fixed with 3\% paraformaldehyde in HBSS with $3 \mathrm{mM} \mathrm{CaCl}$ for 15 minutes on ice and permeabilized with $0.1 \%$ Triton-X in the same buffer. To identify the Golgi, AF488 labeled Griffonia simplicifolia lectin II (Invitrogen, Carlsbad, CA), was incubated with cells. Other Golgi markers used were, rabbit anti- $\beta$ COP (Affinity Bioreagents, Golden, CO.), rabbit anti-giantin, and rabbit anti-Golgin-95 (kind gifts from Dr. Marvin Fritzler, U Calgary, Canada) followed by AF488 labeled goat anti-rabbit IgG (Invitrogen). Anti-Mannosidase-II (Covance, Denver, PA) was used to label medial-Golgi, and was identified by AF488 goat anti-mouse IgG $\gamma 2 \mathrm{~b}$ (Invitrogen). IgM was visualized with AF647 labeled goat anti-mouse IgM (Invitrogen), and DNA was stained with sytox orange (Invitrogen). DiO (Invitrogen) or AF488 labeled annexin V (Invitrogen) were used to stain the cell membrane. In one experiment, cells were treated with nocodazole (Sigma Aldrich, St Louis, MO) at $2.5 \mu \mathrm{g} / \mathrm{ml}$ for two hours before being fixed and stained as described above. Samples were viewed on a Zeiss LSM510 laser scanning microscope, as described $(187,256)$. 


\subsubsection{Electron microscopy}

Cells grown on a polyester permeable support membrane (Corning incorporated, Acton, MA) were fixed with 3\% paraformaldehyde and 2\% glutaraldehyde in HBSS on ice for 15 minutes and washed with $0.05 \mathrm{M}$ glycine in PBS ( $\mathrm{pH} 7.4)$ for 15 minutes. The membrane was cut into wedges and then osmicated in $1 \% \mathrm{OsO}_{4}$ for 4 hours and dehydrated in a graded alcohol series. The membrane was incubated overnight in a 1:1 ethanol: Spurr's low viscosity embedding medium (Electron Microscopy Sciences [EMS], Hatfield, PA) followed by three changes in 100\% Spurr's medium, $3 \mathrm{hrs}$ each. For embedding, the cells in Spurr's medium were cured for 2 days at $80^{\circ} \mathrm{C}$. The polymerized Spurr's medium with cells embedded in it was cut into $75 \mathrm{~nm}$ sections and placed on nickel grids. The sections were treated with $4 \%$ uranyl acetate (Ultrostain I, Leica, Deerfield, IL) in 100\% methanol followed by lead citrate (Ultrostain II, Leica) in acetone to provide contrast to the cellular structures.

IgM aggregates (spherons) were collected from the flask after removing cells and media. The flask's plastic surface to which the spherons had adhered was washed twice with HBSS. After washing, $1 \mathrm{ml}$ of HBSS was added and the flask was scraped with a cell scraper to remove the spherons. The spheron fraction in HBSS was filtered through a $5 \mu \mathrm{m}$ pore filter (Millipore, Billerica, MA) to remove debris and intact cells. The filtrate was spun at 25,000 RPM in an SW-31 rotor for 30 minutes and the supernatant was discarded. The spheron pellet was washed and fixed with $3 \%$ paraformaldehyde and $2 \%$ glutaraldehyde in HBSS on ice for 15 minutes and washed with $0.05 \mathrm{M}$ glycine in PBS ( $\mathrm{pH} 7.4$ ) for 15 minutes. The spherons were incubated in two changes, 10 minutes each, of $0.2 \%$ Aurion BSA-c (EMS) in PBS (pH 7.4) (to reduce background binding by the gold conjugated antibody). Goat anti-mouse IgM antibody conjugated to $25 \mathrm{~nm}$ gold particles (EMS) in $0.1 \%$ BSA-c was added to the spherons and incubated at room temperature for $2 \mathrm{hrs}$. The spherons were post fixed in $2 \%$ glutaraldehyde in PBS (pH 7.4) for 5 minutes and washed with distilled water. Embedding and processing was done as for the cells. All sections were analyzed with a JEOL, JEM-2000 Ex II electron microscope.

\subsubsection{Metabolic labeling and analysis of cell fractions}

Cells were labeled by incubating $2.5 \times 10^{6}$ cells $/ \mathrm{ml}$ in cysteine and methionine free labeling media (Sigma Aldrich) with $100 \mu$ Curie of ${ }^{35} \mathrm{~S}$ labeled cysteine and methionine for $16 \mathrm{hrs}$ at $37^{\circ} \mathrm{C}$ and $5 \% \mathrm{CO}_{2}$. Hybridoma cultures were grown to confluency and then divided into fractions corresponding to media, cells and (plastic adherent) IgM aggregates. Cells were separated from the media by centrifugation at $6000 \mathrm{~g}$ for 15 seconds. Media was collected in separate vials and kept on ice. Cells at 1 X10 $/ \mathrm{ml}$ were lysed in the lysis buffer $(0.05 \mathrm{M}$ Tris $\mathrm{HCl} \mathrm{pH} \mathrm{7.5,} \mathrm{NaCl} 0.15 \mathrm{M}, 0.5 \%$ deoxycholic acid, $0.5 \% \mathrm{NP}-40$ ) and a cocktail of protease inhibitors (Roche, IN) and the cell lysate was collected by centrifugation at $6000 \mathrm{~g}$ for 10 minutes. The pellet was solublized by denaturation in $2 \%$ SDS at $100^{\circ} \mathrm{C}$ for 5 minutes and diluted with lysis buffer to bring the final SDS concentration to $0.1 \%$ to allow for immunoprecipitation. Immunoprecipitation 
was done by adding $10 \mu \mathrm{g} / \mathrm{ml}$ unlabeled goat anti-mouse IgM (Southern Biotech, AL) to all the fractions (cell lysates, media, and NP-40 insoluble fraction) and incubated at $4{ }^{\circ} \mathrm{C}$ on a rotary incubator for $2 \mathrm{hrs}$. 50\% protein A sepharose beads (Sigma Aldrich) were added and incubated at $4{ }^{\circ} \mathrm{C}$ for 30 minutes on rotary incubator. The beads were washed with washing buffer $(0.05 \mathrm{M}$ Tris $\mathrm{HCl} \mathrm{pH} 7.5, \mathrm{NaCl} 0.4 \mathrm{M}, 0.5 \%$ deoxycholic acid, $0.5 \% \mathrm{NP}-40$ ) three times by centrifugation at $6000 \mathrm{~g}$ for 10 seconds. Following the washes, the sepharose beads were divided into two parts. Each part was boiled in either the reducing buffer $(0.125 \mathrm{M}$ Tris $\mathrm{HCl}$ pH6.8, 20\% glycerol, 10\% 2-mercaptoethanol, 4\% SDS) or non reducing buffer (Same buffer without the addition of 2-mercaptoethanol) for 5 minutes at $100^{\circ} \mathrm{C}$. After boiling, the eluates were separated by SDS-polyacrylamide electrophoresis. For reducing conditions $10 \%$ polyacrylamide gels were used. Samples were analyzed under non-reducing conditions on $8 \%$ gels. The gels were run at $150 \mathrm{~mA}$ constant current for about $1.5 \mathrm{hrs}$. The gels were placed on filter paper and dried in a gel drying apparatus (Biorad, Hercules, CA) and visualized by autoradiography. Spherons were collected and purified as described for electron microscopy and run under reducing and non-reducing conditions as above.

\subsubsection{Pulse and chase}

$1.25 \times 10^{7}$ cells/ml were grown in RPMI with $15 \%$ FBS for 16 hrs. The cells were washed twice with $1 \mathrm{x}$ PBS to remove old media. Then cells were starved in incomplete RPMI without methionine and cysteine (Sigma Aldrich), and 1\% dialysed

FBS without ${ }^{35} \mathrm{~S}$, for 40 minutes at $37^{\circ} \mathrm{C}$. The cells were pulsed with $25 \mu \mathrm{Ci}$ of ${ }^{35} \mathrm{~S}$ labeled methionine / cysteine for 15 minutes. The cells were washed twice in cold PBS and chased in complete medium at $37^{\circ} \mathrm{C}$ with excess cold methionine and cysteine for $0,2,4$ and 6 hrs. Cells, media and spherons were collected at the indicated time points. The harvested cells and spherons were treated with lysis buffer. IgM from cell lysates, media and spherons was immunoprecipitated and the material was resolved on SDS-PAGE gels as above.

\subsubsection{Two dimensional gels}

Cells and media were collected and processed as described above. Samples were run in the first dimension under non-reducing conditions in an $8 \%$ gel. The whole strip of gel was cut and incubated in a reducing buffer $(62.5 \mathrm{mM}$ Tris $\mathrm{HCl}(\mathrm{pH} 6.8), 10 \%$ glycerol, 5\% 2-mercaptoethanol, 2\% SDS, $0.05 \%$ bromophenol blue) for 20 minutes. After reduction, the gel strip was placed horizontally atop a slab gel and proteins were separated under reducing conditions on a $10 \%$ gel. In this experiment, cell lysates were either analyzed without chemical cross linking or after chemical cross linking. For cross linking, cells were washed first with PBS (pH7.4) followed by two washes in HEPES buffer ( $\mathrm{pH}$ 8.3). Di-thio-bis succinimidyl propionate (DSP) 0.015\% (in DMSO) was added and incubated for $1 \mathrm{hr}$ on ice and cells were shaken occasionally to avoid pellet formation. The cross linking reaction was stopped by incubating with $1 \mathrm{M}$ glycine for 15 
minutes. Cell lysis and subsequent analysis was performed in the exact manner as done for cells without cross linking.

\subsubsection{Glycosidase treatments}

IgM from cell lysates, media and spheron fractions were immunoprecipitated using protein A sepharose beads. The beads were washed and divided into three parts. One part was untreated; the second was incubated with Endo H (New England Biolabs, Ipswich, MA) and the third was incubated with PNGase F (New England Biolabs). EndoH treatments were performed as recommended by the manufacturer. Briefly, 2x denaturing buffer ( $1 \%$ SDS, $0.08 \mathrm{M}$ DTT) was added to the beads and boiled at $100^{\circ} \mathrm{C}$ for 10 minutes. To the eluate, reaction buffer $(0.05 \mathrm{M}$ sodium citrate $\mathrm{pH} 5.5)$ and Endo $\mathrm{H}$ $(75,000 \mathrm{U} / \mathrm{ml})$ were added and incubated at $37^{\circ} \mathrm{C}$ for $1 \mathrm{hr}$. For PNGase F, the sepharose beads from the cell lysate were incubated in denaturing buffer A $(0.5 \%$ SDS, $150 \mathrm{mM}$ Tris (pH 7.8), boiled at $100^{\circ} \mathrm{C}$ for 2 minutes, cooled and the supernatant containing the IgM was removed. Equal volume of reaction buffer A (4\% NP-40, 3.2mM PMSF, 32mM EDTA) was added followed by PNGase F $(25,000 \mathrm{U} / \mathrm{ml})$ and incubated at $37^{\circ} \mathrm{C}$ for $1 \mathrm{hr}$. The samples were run under reducing conditions. The sepharose beads obtained after immunoprecipitation of the media were incubated in denaturing buffer B $(0.5 \%$ SDS and $100 \mathrm{mM} 2$-Mercaptoethanol) and boiled at $100^{\circ} \mathrm{C}$ for 3 minutes. The supernatant after removing and cooling was added to equal volume of reaction buffer $\mathrm{B}(1 \% \mathrm{NP}-40$, 60mM Tris (pH 8.6), 6mM EDTA, 0.1\% SDS, 0.02 M 2-mercaptoethanol). PNGase $(37,500 \mathrm{U} / \mathrm{ml})$ was added, incubated and samples run under reducing conditions as above.

\subsubsection{Western blotting}

Proteins were transferred from gels to nitrocellulose membrane as described before (257). Alkaline phosphatase labeled goat anti-mouse $\mu$ and goat anti-mouse $\kappa$ antibodies (Southern biotech, Birmingham, AL) were used as secondary reagents according to manufacturers' recommendations. Rabbit anti-human J chain IgG (Nordic Immunology, Tilburg, The Netherlands), was used as the primary antibody to analyze $\mathrm{J}$ chain expression in the hybridomas. The anti $\mathrm{J}$ chain antibody was detected by a HRP conjugated goat anti-rabbit IgG antibody (Sigma Aldrich). Bands were visualized using substrates from the AP color development kit (Biorad) or by chemiluminescence kit (Perkin Elmer, Boston, MA).

\subsection{Results}

\subsubsection{Cells used in this study}

We recently reported on $\mathrm{H} 160.3$, a hybridoma that expresses IgM composed of the VH56R+Vк38c H-L pair. This hybridoma exhibits multiple self-reactivities yet it is abundantly represented in peripheral tissues (e.g. the spleen) of non-autoimmune mice. 
Here, we wished to examine the mechanism that allows this type of B cell to escape tolerance induction in the bone marrow. The escape from tolerance correlates with the intra-Golgi accumulation of IgM and reduced surface IgM receptor expression. The IgM $\mathrm{Ab}$ secreted from the hybridoma binds to dsDNA, PS, MBP, thyroglobulin, cytochrome c, histones, insulin, and beta-galactosidase in a direct ELISA (127). For comparison, we chose to analyze the L55 hybridoma. This cell line expresses the same H chain, VH56R, and was immortalized using a similar procedure as H160.3. However, L55 differs from H160.3 in expressing the V $\lambda \times$ L chain. In contrast to H160.3, L55 secretes IgM that binds weakly to MBP but not to the other antigens tested (228).

One striking difference between the H160.3 and L55 hybridomas was their intracellular IgM distribution (Figure 3.1A, C). In H160.3, a substantial amount of IgM accumulated in a distinct and restricted area of the cell, although additional IgM was found elsewhere in the cytoplasm. In contrast, there was essentially no IgM in the corresponding area in L55 cells and the cytoplasmic IgM was distributed throughout the cell (Figure 3.1B, D). For orientation, our reagents also identified the nucleus by means of DNA staining and the plasma membrane by means of annexin $\mathrm{V}$ binding. As before (256), we again noted that the H160.3 cells also display large IgM clusters at the cell surface.

Because the different intracellular IgM distributions arose in cells expressing the same $\mathrm{VH}$, we surmised that the intracellular IgM accumulation reflects the use of the $\mathrm{V} \kappa 38 \mathrm{c} \mathrm{L}$ chain. To test the idea that $\mathrm{V} \kappa 38 \mathrm{c}$ determines intracellular accumulation, we searched for another cell line that expresses the Vk38c L chain. Therefore, we examined the IgM distribution in 38C-13, a B cell lymphoma expressing Vк38c $(258,259)$, and the control B cell lymphoma, WEHI 231. Consistent with a Vк38c contribution to the IgM accumulation, 38C-13 cells showed increased IgM intracellularly (Figure 3.1E), whereas, in WEHI 231 cells, IgM was distributed in a more peripheral manner (Figure 3.1F). Thus, immunofluorescence detection of IgM supported the idea that the Vк38c L chain favors IgM accumulation. We infer that intracellular accumulation of IgM derives an important contribution from the VK38c L chain.

\subsubsection{The VH56R+Vк38c IgM accumulates in the Golgi}

Few, if any, reports have demonstrated the accumulation of IgM within the Golgi. Therefore, we selected a set of Golgi-specific markers to examine the degree of colocalization with the IgM in the H160.3 and L55 hybridomas (Figure 3.2). We used antibodies to Golgi-associated proteins to define a space within the cell that contains the Golgi, in order to ask two related questions: 1 . What is the fraction of total IgM that is located within that cellular space, and 2. What is the degree of overlap between the IgM and the various antibodies to the marker proteins? Typical cells used in the analysis are shown in Figure 3.2, and overall quantitation of intracellular distribution and colocalization with markers is presented in Table 3.1. The markers that we used were: giantin, a cis-Golgi associated membrane protein $(197,260)$; golgin-95, a protein with roles in Golgi structure and function $(261,262) ; \beta$-COPII, a protein involved in the ER to 

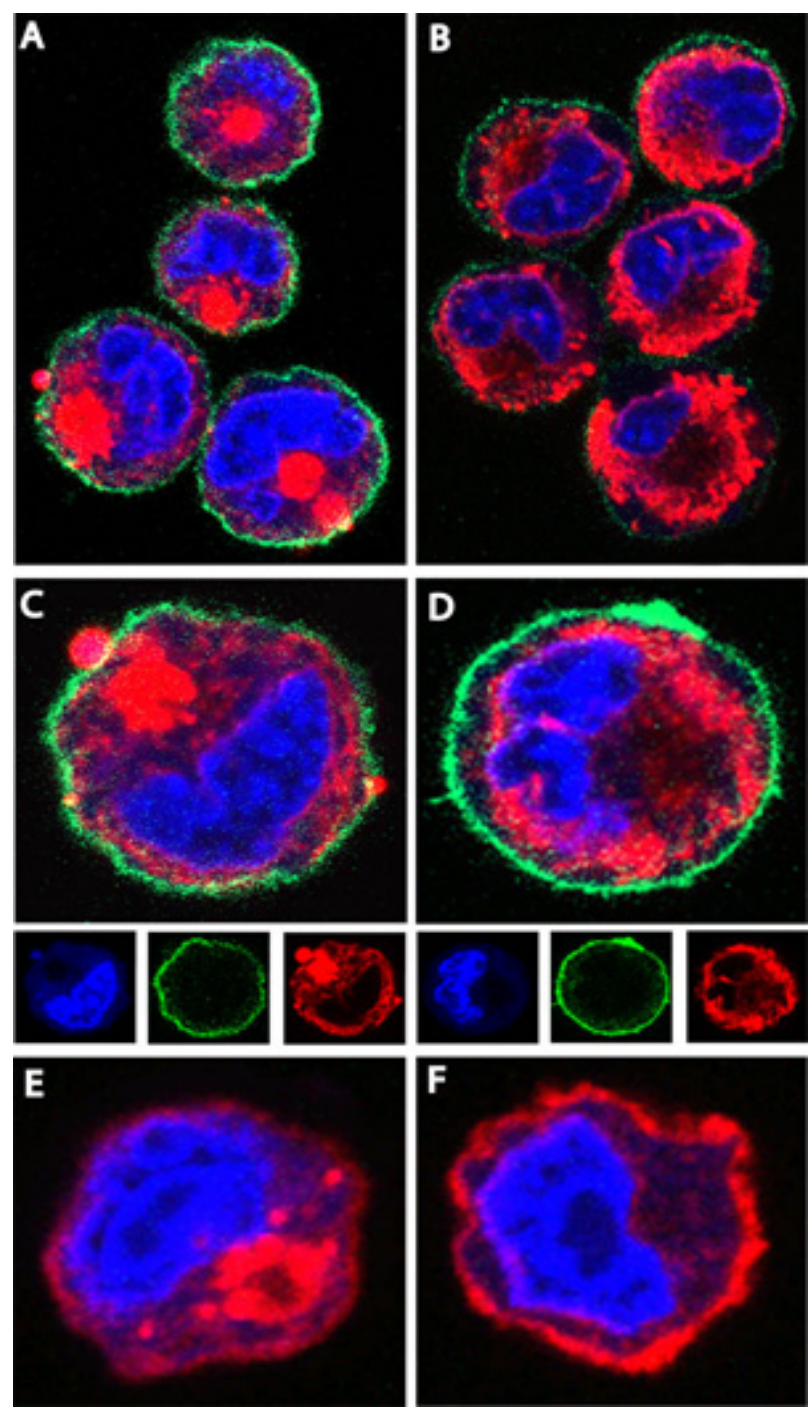

Figure 3.1 Distribution of IgM in B cell hybridomas and lymphomas.

Hybridomas H160.3 (A and C) and L55 (B and D) were fixed, permeablized and incubated with AF647 goat anti-mouse IgM (red), AF488 Annexin V (green) and Sytox orange (blue). IgM accumulates intracellularly in the H160.3 cells (A). In contrast, IgM in L55 cells is distributed throughout the cytoplasm. (B). Magnified images of H160.3 (C) and L55 (D). IgM aggregates are present at the cell membrane (Panels A and C). 38C-13 lymphoma cell (E) and WEHI-231 cell (F) (treated as above) were stained with AF647 goat anti-mouse IgM and Sytox orange. IgM accumulates intracellularly in 38C13 cells (E) whereas IgM in WEHI-231 cells (F) has a more peripheral distribution. 
Figure $3.2 \operatorname{IgM}$ accumulates in the Golgi in hybridomas.

H160.3 ( or an alternate subclone: H40) (Left panels) and L55 cells (Right panels) were incubated with Golgi markers followed by secondary antibodies (green), AF647 goat anti-mouse IgM (red), and Sytox orange (blue). Each image has three panels, the top left is a $2 \mathrm{D}$ representation, the top right and the bottom left are the projected images. The area indicated by a white square shows a magnification of the Golgi. The Golgi (green) and intracellular IgM (red) split images are represented next to the Golgi magnification. H40 (A) and L55 (B) were stained with rabbit anti-Giantin and visualized by AF488 Goat anti-rabbit IgG (green). IgM surrounds the Giantin and the anti-Giantin stains the center of the Golgi more intensely. The projected images show IgM adjacent to the Giantin and the co-localization can be seen as fine yellow lines. H40 (C) and L55 (D) were stained with rabbit anti-Golgin95 followed by AF488 Goat anti-rabbit IgG (green). Rabbit anti- $\beta$ COP was incubated with H160.3 cells (E) and L55 cells (F) followed by AF488 Goat anti-Rabbit IgG (green) to visualize $\beta$ COP. The IgM and the $\beta$ COP stains co-localize partially. The greatest degree of overlap between IgM and a Golgi marker occurred when H160.3 cells (G) were stained with AF488 GS Lectin II (green). Lectin II stains free terminal GlcNAc residues and free GlcNAc residues are in the medial-Golgi. Thus it is likely that the intracellular accumulation of IgM in H160.3 cells is localized to the medial-Golgi. Mouse anti-Mannosidase II antibodies, a medial-Golgi marker, were incubated and visualized with AF488 Goat anti-mouse IgG $\gamma 2 \mathrm{~b}$ (green) in $\mathrm{H} 40$ cells (I). In these cells, Mannosidase II stains as fine spots taking a granular appearance and the IgM co-localizes with this marker to a considerable extent. IgM in L55 cells (B, D, F, H and J) while being distributed in the ER, is not present in the Golgi and therefore does not show co-localization with any of the Golgi markers used here. 

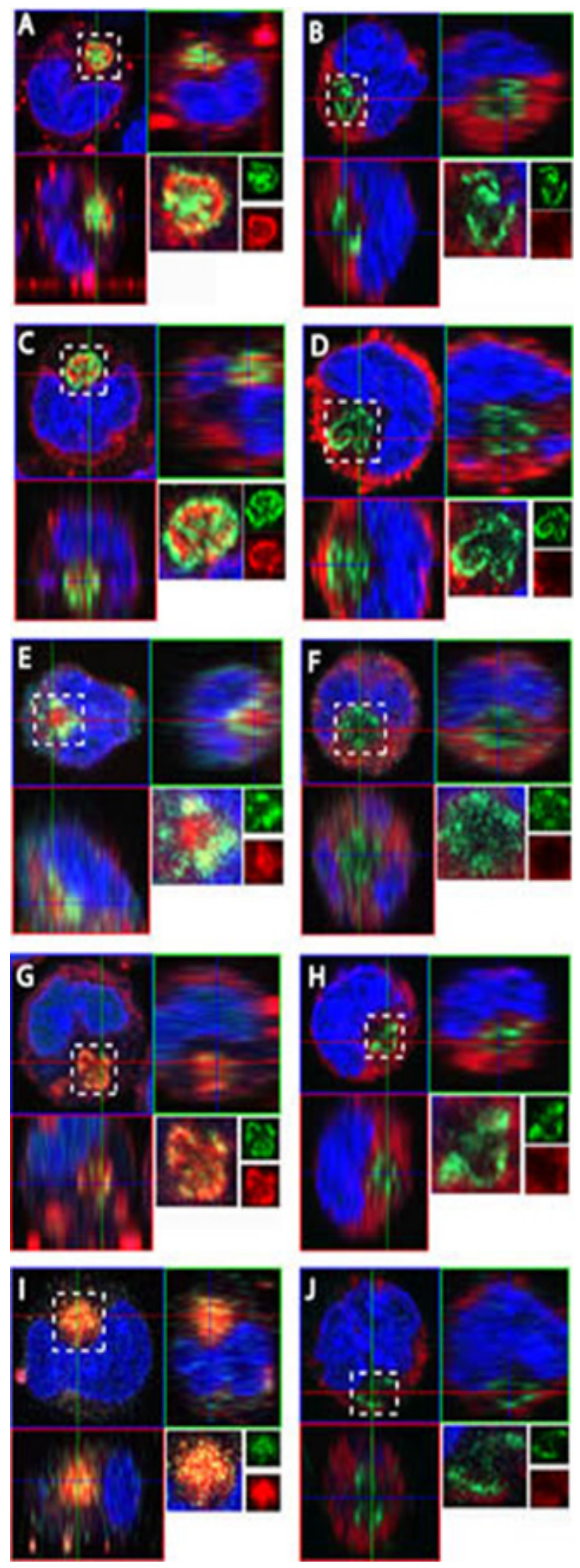
Golgi shuttling of cargo $(263,264)$; lectin II, a protein with binding specificity for the terminal GlcNAc moiety (265); and Golgi mannosidase II, a resident enzyme that removes $\alpha 1,3$ - and $\alpha-1,6-$ mannose residues converting the GlcNAcMan $_{5}$ to GlcNAcMan $_{3}$ and serves to identify the medial Golgi (266).

We examined complete three-dimensional reconstructions of the hybridoma cells and we show images in the three orthogonal planes defined by $x y, x z$, and yz coordinates (Figure 3.2). In addition, the area most closely corresponding to the Golgi (indicated by the boxed area) is shown magnified and by using separate colors for IgM and the marker antibodies. These images confirmed the profound difference in IgM distribution between the two hybridomas. A considerable portion of cellular IgM in H160.3 (or its surrogate H40) was present at or near the Golgi area indicated by the markers, whereas essentially no IgM co-localized with the markers in L55. The five different markers had different degrees of co-localization with the IgM in H160.3 (or H40), although none of the markers completely co-localized with the IgM in this compartment. Anti-giantin (Figure 3.2A) and anti-Golgin-95 antibodies appeared interlaced and partially overlapping with the IgM (Figure 3.2C), whereas antibodies to $\beta$-COP typically were found to surround the IgM (Figure 3.2E). The greatest degree of overlap was observed between the H160.3 IgM and lectin II (Figure 3.2G) and between IgM and antibodies to mannosidase II (Figure 3.2I).

To get a more quantitative answer to our questions, we computed the accumulation of $\operatorname{IgM}$ in and around the Golgi and determined the percent overlap between the IgM and the various marker antibodies (Table 3.1). We used areas of intense staining with the marker antibodies to constrain the space containing the Golgi and compute the relative proportion of H160.3 or L55 IgM located within the Golgi as compared to elsewhere in the cell. In addition, we determined the extent of overlap between the H160.3 IgM in the Golgi and the various Golgi markers.

Table 3.1 Percent IgM in Golgi area compared to rest of the cell and percent colocalization of IgM with different Golgi markers.

\begin{tabular}{lccccr}
\hline Markers & Giantin & Golgin-95 & $\beta$-COPII & Lectin II & Mannosidase II \\
\hline $\begin{array}{l}\text { H160.3 } \\
\text { \% IgM in }\end{array}$ & $18 \pm 2 \%$ & $17 \pm 2 \%$ & $18 \pm 1 \%$ & $16 \pm 1 \%$ & $22 \pm 2 \%$ \\
Golgi & & & & \\
$\%$ & $32 \pm 10 \%$ & $22 \pm 5 \%$ & $30 \pm 24 \%$ & $71 \pm 5 \%$ & $66 \pm 4 \%$ \\
Co-localization & & & & & \\
$\begin{array}{l}\text { L55 } \\
\text { \% IgM in }\end{array}$ & $<2 \pm 0.7 \%$ & $<1 \pm 0.5 \%$ & $<0.05 \pm 0.003 \%$ & $<1 \pm 0.5 \%$ & $<0.5 \pm 0.04 \%$ \\
Golgi & & & & & \\
\hline
\end{tabular}


Each of the Golgi markers gave a similar Golgi outline, as the values of H160.3 IgM within the Golgi fell within the narrow range of 16 to $22 \%$ of total IgM (Table 3.1). In contrast, less than $2 \%$ of IgM was found in this location in the L55 hybridoma. The percent overlap between H160.3 IgM and the marker antibodies was 22\% for golgin-95, and $30-32 \%$ for giantin and $\beta$-COP (Table 3.1). More extensive overlap was found with antibodies to mannosidase II (66\%) and for the fluorescent lectin II (71\%) (Table 3.1). Free terminal GlcNAc moieties, targets of Lectin II, are concentrated in the medial Golgi and mannosidase II is primarily present in the medial Golgi with a smaller percentage distributed in the trans-Golgi (267). Therefore in sum, we conclude that H160.3 cells accumulate $\operatorname{IgM}$ in the Golgi compartment and that the accumulation involves parts of the cis-Golgi and most of the medial and trans-Golgi compartments. In L55 control cells, very small to negligible amounts of IgM are detectable within these areas of the secretory compartment (Table 3.1). Because we observed only an incomplete overlap between the H160.3 IgM and the Golgi markers, we decided to alter the architecture of the Golgi by nocodazole treatment and to compare the distribution of anti-golgin-95 and $\operatorname{IgM}$ in H160.3 and L55 cells (Figure 3.3). The organization of the Golgi complex is interdependent with the microtubule network and the disruption of microtubules by nocodazole leads to a "scattering" of the Golgi fragments (268). In cells treated with nocodazole for 2 hours, we could observe between 10 and 15 organelle fragments. In H160.3 cells, most of the golgin-95 containing Golgi vesicles also reacted with anti-IgM antibodies (Figure 3.3A), whereas most of the anti-IgM in L55 cells did not co-localize with anti-golgin-95 (Figure 3.3D). This confirmed our inference that H160.3 IgM is bound by a membrane network that behaves in a manner consistent with the Golgi complex.

\subsubsection{Distribution and separation of $\operatorname{IgM}$ aggregates from the cell}

In an effort to more carefully examine the IgM distribution in $\mathrm{H} 160.3$ cells, we grew the hybridoma on coverslips and prepared serial sections in the xy plane (Figure 3.4A). One such series of images is shown in Figure 3.4B-H. It can be seen that in this cell, the Golgi was near the midsection of the stack adjacent to the cell's nucleus (Figure 3.4E-G). In addition, we observed IgM clusters at the cell surface. The last optical section (Figure 3.4H), grazing the surface of the coverslip, revealed spherical aggregates of IgM that were scattered at variable distances from the cell's attachment site. We refer to these IgM deposits that as "spherons" due to their shape. The density of spherons along the glass surface decreased with increasing distance from the cell, suggesting that the cell was the likely source of the IgM deposits (Figure $3.4 \mathrm{H}$ ).

To test whether the spherons are bound by a membrane while they remain attached to the cell surface or after they detach and settle on the glass surface, we visualized the plasma membrane by staining with $\mathrm{DiO}$, a lipophilic carbocyanine dye. This dye inserts into phospholipid bilayers, and labels the external and internal membranes of the cell (269). This stain was applied to living cells and the binding was visualized without fixation or other harsh treatments. Anti-IgM antibodies labeled with AF647 were added to the cells in a physiological buffer and the cells on the coverslips 

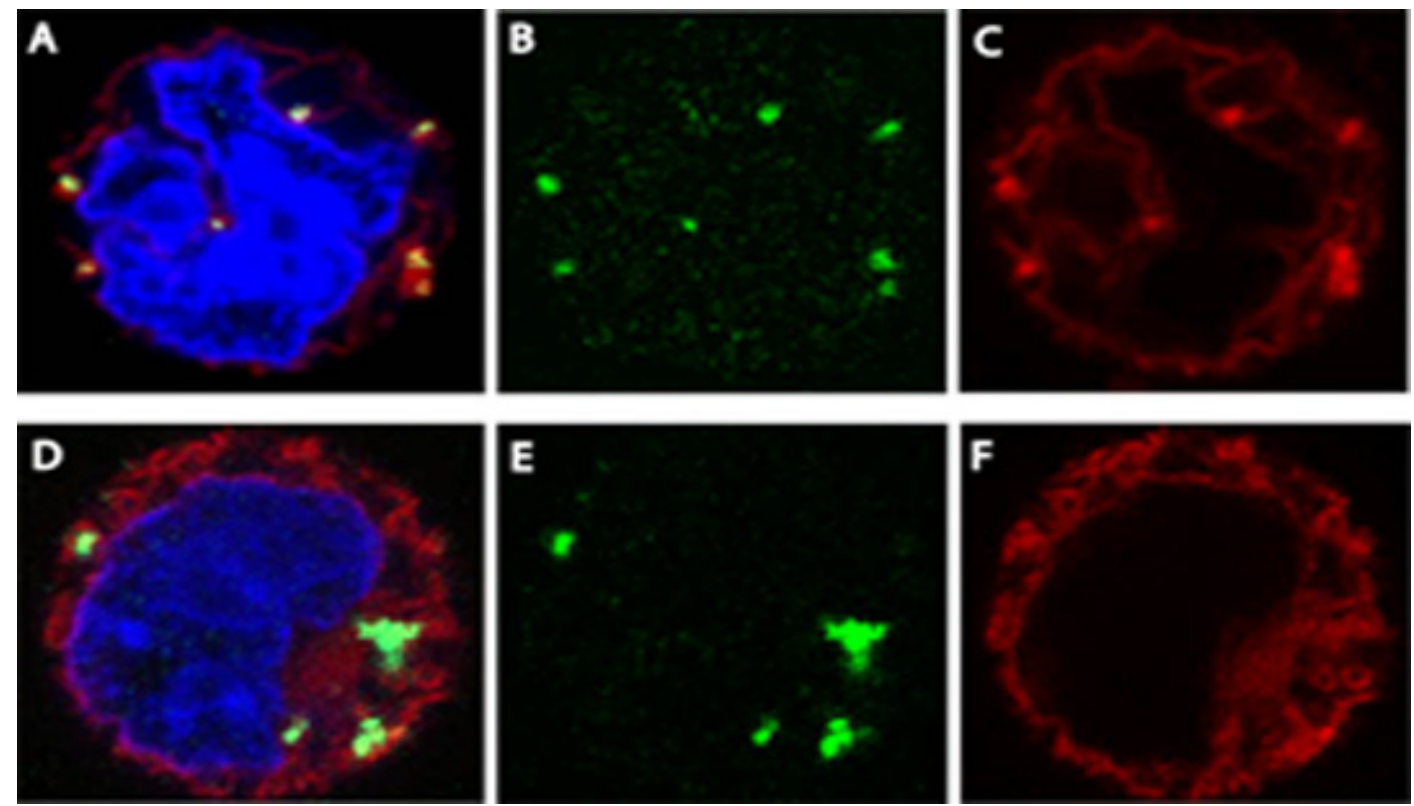

Figure 3.3 Golgi fragments after nocodazole treatment.

H40 cells (A) and L55 cells (D) were treated with $2.5 \mu \mathrm{g} / \mathrm{ml}$ nocodazole for two hours, fixed, permeablized and incubated with rabbit anti-Golgin-95 for $3 \mathrm{hrs}$. This was followed by AF488 Goat anti-rabbit IgG (green), AF647 goat anti-mouse IgM (red), and Sytox orange (blue). Panel (A) shows the fragmented Golgi distributed in $\mathrm{H} 40$ cells that react with anti-golgin-95 (B) and anti-IgM (C). Antibody co-localization results in yellow fluorescence (A). Panels (B) and (C) are the corresponding split images of panel (A). In contrast, the IgM (F) in L55 cells does not co-localize with anti-golgin-95 marking the fragmented Golgi (E). 
Figure 3.4 Analysis of hybridomas and spherons by microscopy.

H160.3 cells were grown on cover slips for various times up to two days. The cells grown overnight on the cover slips were fixed, permeablized and stained with AF647 goat antimouse IgM (red), and Sytox orange (blue). A stained cell on the cover slip was photographed beginning from the top (Slice1) and the subsequent sections towards the bottom were taken at intervals of $0.88 \mu \mathrm{M}$ thickness (Sections 2 through 7) (A). Section 7 passes through the spherons that have settled on the cover slip. The top of the cell (B) shows faint IgM and nuclear stain. As the cell is sectioned $(\mathrm{C}-\mathrm{G})$, the intracellular IgM accumulation is noticeable. The last section nearest to the cover slip $(\mathrm{H})$ shows spherons that have settled around the cell. The cell is not seen because the section grazes the cover slip. A dashed white circle represents the place where the cell rests. H40 cells grown for two days on the cover slip were stained with DiO (green), AF647 goat anti-mouse IgM (red), and Sytox orange (blue) without fixation and permeablization ( $\mathrm{I}-\mathrm{O})$. Here only the IgM spherons at the cell membrane were stained, the cellular IgM was not accessible to the anti-IgM. The cell was sliced beginning at the top (I) and subsequent sections were towards the cover slip $(\mathrm{J}-\mathrm{N})$. The spherons located on the cell membrane are not surrounded by a lipid membrane $(\mathrm{I}-\mathrm{N})$ and neither do the spherons that have settled around the cell on the cover slip (O). 


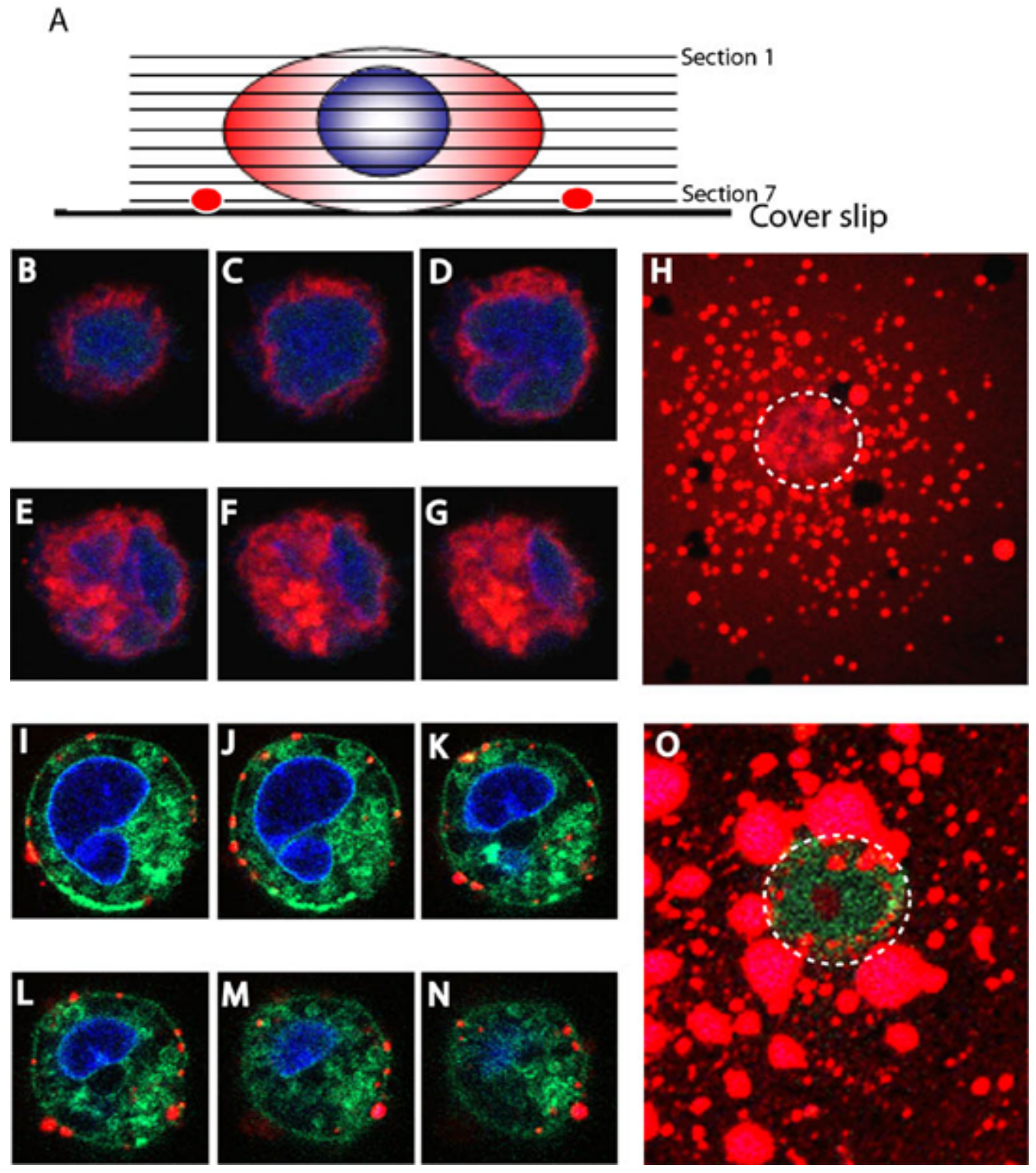


were observed under the microscope (Figure 3.4I-O). Cells incorporating DiO revealed the presence of numerous surface IgM clusters that were mostly devoid of DiO staining (Figure 3.4I-N). On the coverslip, spherons lacked DiO staining, suggesting that they are free of a DiO-reactive coat (Figure 3.4O).

\subsubsection{Ultrastructural features of spherons}

To gain a closer view of the IgM aggregates at the cell surface and after their deposition on the surface of the polyester membrane in the growth chamber, we prepared cells and spherons for electron microscopy. We observed spherons that were attached to the cell surface (Figure 3.5A and B) and noted that spherons consist of a relatively uniform, grainy material and that the cell provides a relatively flat anchoring surface for the spherons. The spheron when magnified shows an approximately circular contour and consisted of uniformly distributed amorphous material. Along their edges, spherons did not have any distinct boundary layer, although their outline ranged from being relatively smooth to showing partial disaggregation (Figure 3.5B). The spheron shown in Figure $3.5 \mathrm{~B}$ had a diameter of $1.53 \mu \mathrm{m}$. Collected spherons that had settled to the surface of the culture vessel tended to disaggregate during the purification method used, such that they revealed further details of their internal structure (Figure 3.5C). Near the edges of these aggregates, the internal structure was revealed as consisting of branched chains of antiIgM-reactive material (Figure 3.5D).

\subsubsection{Characteristics of spheron production by H160.3 hybridoma}

To measure the rate at which cells produce spherons, we grew H160.3 cells for defined times on coverslips and used light microscopy to observe the generation of new deposits (Figure 3.6A). To confirm that the visible deposits corresponded to spherons, we processed the coverslips for immunohistochemistry (Figure 3.6B). The rate of secretion of spherons produced by the cells was analyzed by incubating cells on cover slips for about 16 hours and counting the spherons after staining with anti- $\mu$ antibody. This allowed us to estimate the rate of spheron production as 13 spherons/cell/hour. This is certainly an underestimate because some spherons are likely to be dislodged from the glass surface by the various washing and incubation steps in our procedure. Moreover, it is likely that some spherons are displaced by Brownian motion from the cell and its immediate surroundings. Nevertheless, this estimate suggests that the production of spherons is a rather efficient mechanism to expel $\operatorname{IgM}$ from the cell. Microscopy also allowed us to measure the size of spherons, and we found that most spherons measured approximately $1 \mu \mathrm{m}$ in diameter, although spheron size could reach and exceed $2 \mu \mathrm{m}$ (Figure 3.6C). To confirm that the spherons contained IgM, we prepared the spherons by scraping them from the surface of the culture flask and collecting them by centrifugation. Gel electrophoresis of the enriched spheron fractions revealed that the major proteins contained in this fraction were the IgM H and L chains (Figure 3.6D). 

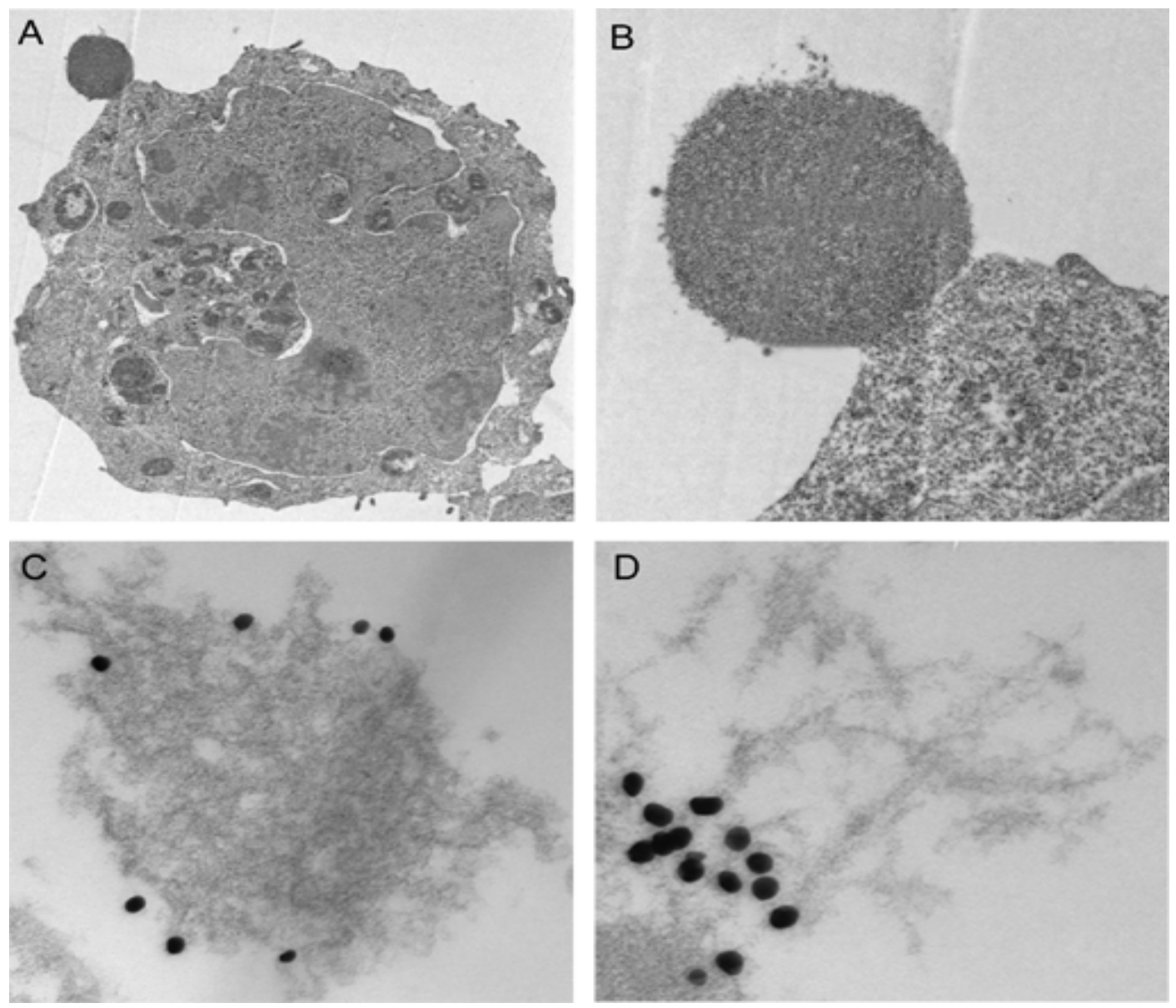

Figure 3.5 Ultrastructure of the spherons.

Hybridoma H160.3 cells were grown on a polyester permeable support and stained with uranyl acetate and lead citrate. $75 \mathrm{nM}$ sections were viewed on nickel grids. A spheron of about $1.5 \mu \mathrm{M}$ is present on the cell at the top left (A). A magnified image of the spheron is shown (B). The spheron appears to be made up of uniformly distributed granular material. There is no visible membrane covering the spheron. The spheron's outer edge is rough with an uneven appearance. Spherons that had settled on the tissue culture flasks were scraped, filtered, centrifuged and incubated with Goat anti-mouse IgM antibodies conjugated to $25 \mathrm{nM}$ Gold particles. Spherons lose their spherical shape during the purification process. Gold-conjugated anti-IgM antibodies bind to the outer edge of a purified spheron $(\mathrm{C})$. Spherons disintegrate into what appear to be protein fibrils and the anti-IgM bind these fibrils well (D). 

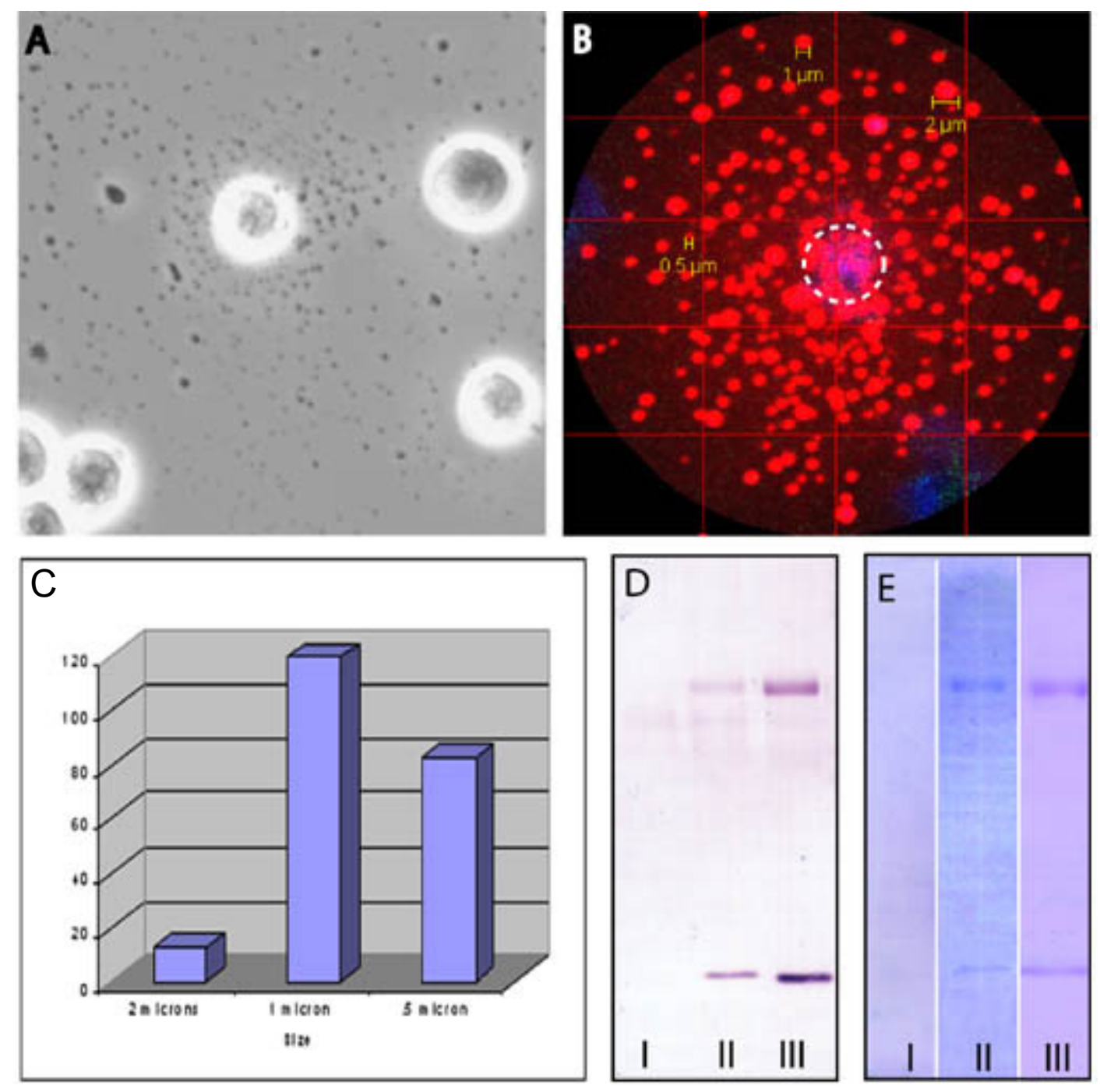

Figure 3.6 Rate of secretion, size and composition of the spherons.

H160.3 cells were grown for $16 \mathrm{hrs}$ on a cover slip and the spherons that had settled around the cells were viewed under a light microscope (A) or were stained with AF647 goat anti-mouse IgM (red), and Sytox orange (blue) (B). Sytox orange was included to distinguish apoptotic bodies. The size of the spherons varied from $0.5 \mu \mathrm{M}$ to $2 \mu \mathrm{M}(\mathrm{C})$. Spherons were purified and run electrophoretically under reducing conditions on a $10 \%$ SDS-polyacrylamide gel. Western blot was probed with alkaline phosphatase conjugated Goat anti-Mouse IgM and Goat anti-mouse $\kappa$ (D). L55 cells do not produce spherons (DI), while H160.3 (D-II) and H40 (D-III) extrude spherons which are composed of the $\operatorname{IgM~Hc}$ and the $\kappa$ Lc. Corresponding gels stained with coomassie blue are shown $(\mathrm{E}-\mathrm{I}$, II and III). 


\subsubsection{Analysis of IgM under non-reducing conditions}

It is highly unusual for B cells to extrude antibody in the form of spherons. We therefore wanted to analyze all the $\operatorname{IgM}$ fractions, the secreted IgM in the supernatant, and IgM in the spherons extruded by the cells in order to learn about their production. The intracellular IgM was analyzed to determine the characteristics of the Ig that is retained inside the cell. Hybridomas H160.3, L55 and H40 were examined. Hybridomas expressing the VK38c Lc show that intracellular IgM is predominantly composed of higher order polymers. There are lesser amounts of IgM that run at about $210 \mathrm{kDa}$ and $\sim 110 \mathrm{kDa}$. These presumably are monomers and hemimers. $\mathrm{H}_{2} / \mathrm{L}_{2}$ make up the monomer. The size of the $\mathrm{H}_{2} / \mathrm{L}_{2}$ alone is approximately about $190 \mathrm{kDa}[2 \mathrm{x}(70+25)]$. Asparagine linked glycosylation of $\mathrm{Hc}$ adds about $20 \mathrm{kDa}(2 \times 10(5 \times 2))$ to the monomer. Thus the monomer has an approximate size of about $210 \mathrm{kDa}$. The hemimers by the same calculation are about $105 \mathrm{kDa}[1 \times(70+25+10)]$. (Figure 3.7A, Lane 1 and Figure 3.7C, Lane 1). The intracellular IgM in L55 cells is quite similar: L55 cells also show mostly higher order polymers inside the cells. There are low amounts of monomers and hemimers.

Hybridoma 160.3 secrete soluble IgM. However, surprisingly, they almost exclusively secrete monomers and hemimers instead of polymers (Figure 3.7A, Lane 2 and Figure 3.7C, Lane 2). Normally B cells secrete IgM as pentamers (74) or hexamers and the presence of the hemimers and monomers is highly unusual.. L55 cells secrete polymers almost exclusively. (Figure 3.7B, Lane 2). Spherons entirely consist of IgM polymers in the Vк38c Lc expressing cells (Figure 3.7A, Lane 4 and Figure 3.7C, Lane 4). Spherons were not detected in L55 cells (Figure 3.7B, Lane 4). Since we had observed

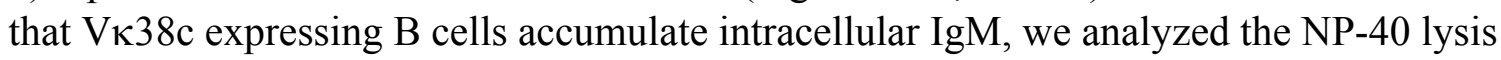
buffer insoluble fraction to check whether there is NP-40 insoluble intracellular IgM. Normally lysis buffers having $0.5 \% \mathrm{NP}-40$ are sufficient to solublize luminal proteins, but this buffer is unable to solublize IgM in the form of Russell bodies $(106,253)$. Vк38c expressing hybridomas do not have Russell bodies (usually located in the ER), but here too there is a NP-40 insoluble IgM fraction in the form of polymers (Figure 3.7A, Lane 3 and Figure 3.7C Lane 3). The NP-40 insoluble fraction in H160.3 and H40 cells could represent IgM that has been modified in some way representing the earlier stages of spherons. There is no NP-40 insoluble IgM in the $56 \mathrm{R}+\mathrm{V} \lambda \mathrm{x}$ cells (Figure 3.7B, Lane 3).

\subsubsection{IgM fractions analyzed under reducing conditions}

The reduced $\mu \mathrm{Hc}$ migrates at about $70 \mathrm{kDa}$ in all three hybridomas (Figure 3.7D, 3.7E, 3.7F - Lane 1). The secreted IgM in all three hybridomas has a slightly higher molecular weight which corresponds to about $73 \mathrm{kDa}$ approximately. The slight upward shift is due to the additional carbohydrate moieties added to the $\mathrm{Hc}$ in the Golgi just before IgM secretion (270) (Figure 3.7D-F Lane 2). Analysis of IgM in the Vк38c expressing hybridomas in the NP-40 insoluble fraction (Figure 3.7D and 3.7F Lane 3) shows presence of IgM. This fraction appears to co-migrate with the secreted IgM. However there could be an error in the assumption and the insoluble fraction might run 
Figure 3.7 Resolution and identification of intracellular IgM, secretory IgM and spheron IgM in hybridomas.

Three IgM producing hybridomas H160.3, L55 and H40 were metabolically labeled for $16 \mathrm{hrs}$ with ${ }^{35} \mathrm{~S}$ cysteine and methionine. Cells were lysed in a $0.5 \%$ Nonidet P-40 buffer in the presence of protease inhibitors. Proteins were immunoprecipitated with Goat antimouse IgM antibodies and protein A-Sepharose and resolved on 8\% SDS-polyacrylamide gels under non-reducing conditions $(\mathrm{A}-\mathrm{C}$ ) or on $10 \%$ gels under reducing conditions (D $-\mathrm{F}$ ). The three predominant bands in the cell lysates (Lanes 1, 5,9) in the non-reduced gels (A, B and C) migrated to a position corresponding to an IgM polymer, monomer of approximately $200 \mathrm{kDa}\left(\mu_{2} \mathrm{~L}_{2}\right)$ and a hemimer $105 \mathrm{kDa}(\mu \mathrm{L})$. H160.3 and H40 secreted predominantly $\mu_{2} \mathrm{~L}_{2}$ and $\mu \mathrm{L}$ whereas L55 secreted mainly polymers (Lanes $2,6,10$ ). The NP-40 insoluble fraction contained only IgM polymers in H160.3 and H40 while L55 had little or no NP-40 insoluble material (Lanes 3, 7, 11). The purified spherons extruded by H160.3 and H40 were composed of IgM polymers. L55 cells did not produce spherons (Lanes 4, 8, 12). All fractions resolve into $\mu \mathrm{Hc}$ and $\kappa \mathrm{Lc}$ under reducing conditions in H160.3 cells and H40 (Lanes 13 -16 and 21 - 24). In L55 cells the cellular fraction (Lane 17) and the secreted fraction (Lane 18) resolve in to the $\mu \mathrm{Hc}$ and the $\lambda \mathrm{Lc}$ while the other two lanes representing the NP-40 insoluble fraction and the spherons do not have IgM H or Lc (Lanes 19 and 20). Analyses of J chain content in the hybridomas by Western blot (G). Secreted IgM from H40 (Lane 25), from L55 (Lane 26), Human IgM (Lane 27) and spherons from H 40 (Lane 28) were resolved on a 10\% SDS-polyacrylamide gel and transferred to nitrocellulose blots. Blots were incubated with Rabbit anti-human J chain antibody and probed with HRP conjugated Goat anti-Rabbit IgG. The J chain was visualized by chemiluminescence. $\mathrm{H} 40$ secrete mainly $\mu_{2} \mathrm{~L}_{2}$ and $\mu \mathrm{L}$ and therefore $\mathrm{J}$ chain was not detected in the secreted IgM (Lane 25). L55 cells secrete IgM polymers and J chain was observed (Lane 26). The Human IgM used as a positive control has a prominent J chain band (Lane 27). Spherons from H40 contain IgM polymers that incorporate the $\mathrm{J}$ chain (Lane 28). The corresponding gel stained by coomassie blue is shown (Lanes 29-32). All lanes show either $\kappa$ or $\lambda$ Lc. The J chain could not be detected in $\mathrm{H} 40$ cells because the cells secrete incompletely assembled IgM. 

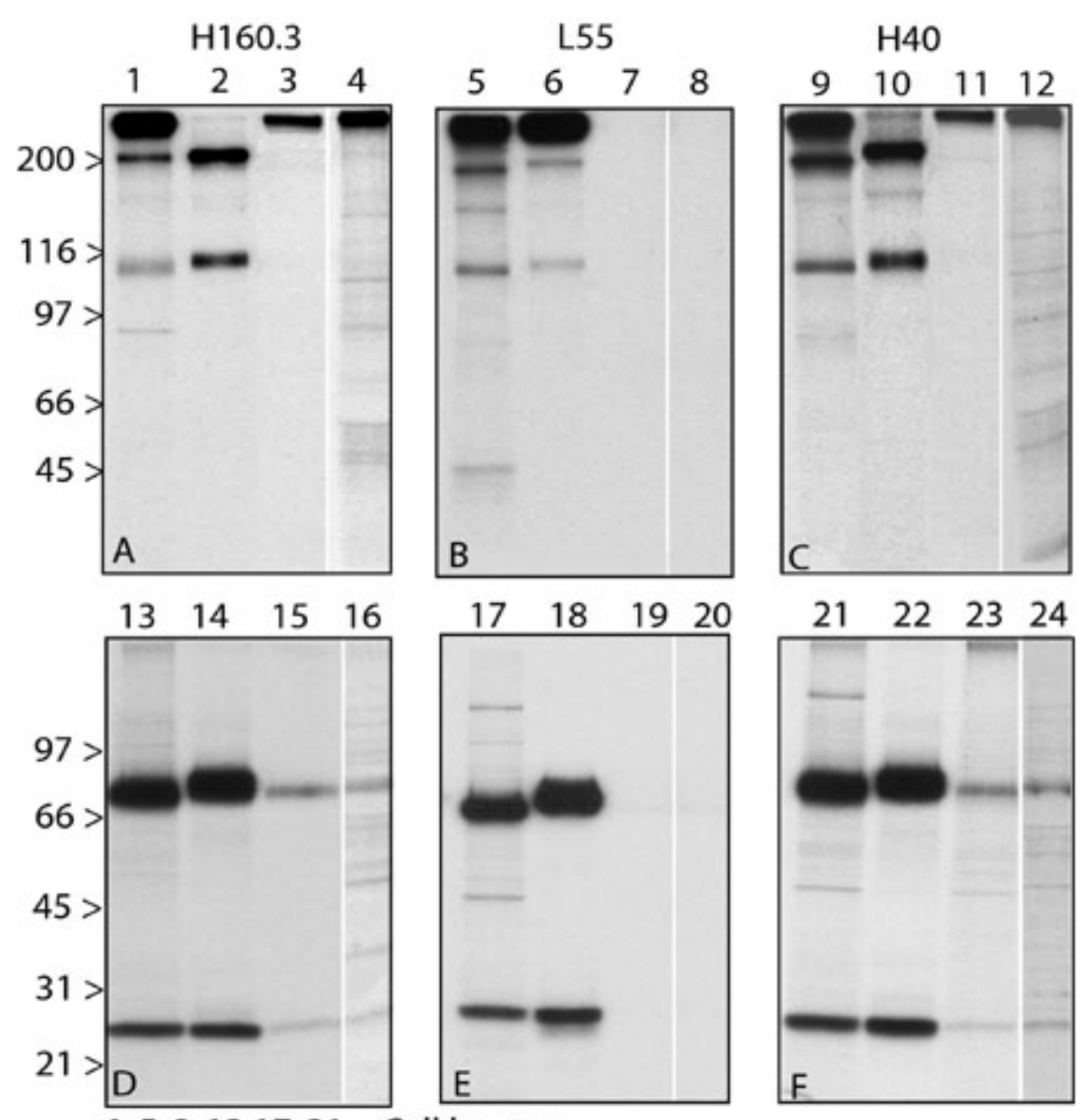

$1,5,9,1317,21$ - Cell lysates

$2,6,10,14,18,22$ - Supernatants

$3,7,11,15,19,23$ - NP-40 insoluble fractions

4, 8, 12, 16, 20, 24 - Spherons

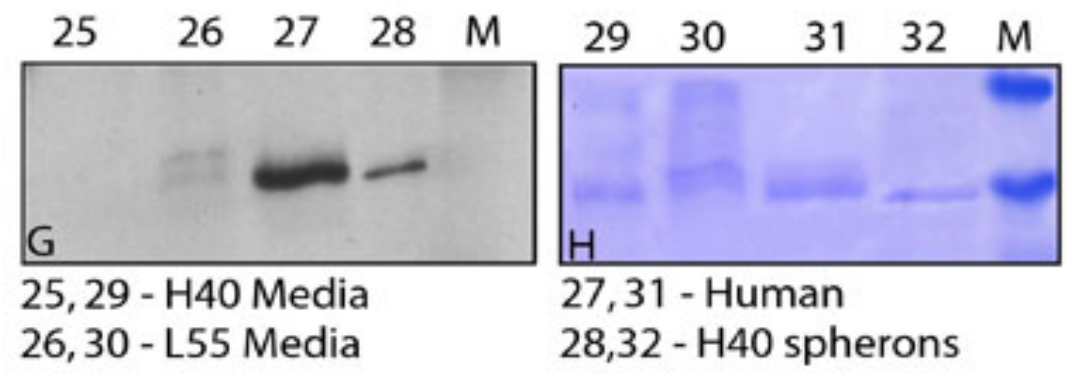


parallel to the intracellular IgM. Thus this fraction could represent the insoluble IgM located either in the Golgi or in the ER. Spherons analyzed under reducing conditions show the $\mu \mathrm{Hc}$ and the $\kappa \mathrm{Lc}$ (Figure 3.7D and 3.7F, Lane 4).

\subsubsection{Analysis of IgM in two dimension}

Examining IgM separately either at reducing or non-reducing conditions in one dimension yields only partial information about the approximate size and composition of the various fractions of the antibody. To analyze the composition of protein multimers that are stabilized by di-sulfide bonds, we carried out 2D gel electrophoresis. In this technique a non-reducing dimension was run on SDS-PAGE and separate lanes were excised, reduced and run out under reducing conditions. This method allowed us to examine $\mathrm{H} / \mathrm{L}$ associations in anti- $\mu$ immunoprecipitation. In addition, we compared proteins that are non-covalently linked with IgM in the different compartments. H160.3 cells in the first dimension (Figure 3.8A1) show IgM polymers intracellularly along with smaller amounts of monomers and hemimers. When these fractions are run at the second dimension, it becomes apparent that the polymers consist of the Hc and the Lc only (Figure 3.8A2). Another band that runs slightly lower is likely the Hc present in the ER because it matches the size of the Hc but there is no corresponding Lc there. Addition of a soluble chemical cross linker, (DSP), before cell lysis probably causes the chaperones that bind the IgM Hc to remain attached even after cell lysis. The Hc bound to chaperones show as additional spots above and below the Hc fraction in both hybridomas tested (Figure 3.8C2 and D2). The spot immediately above the Hc is likely the chaperone GRP94 $(252,271,272)$. When the cross linker was not used, the chaperones could separate from the monomers, hemimers or the glycosylated Hc. Hybridomas H160.3 secrete incompletely assembled IgM forms (Figure 3.8E1) instead of polymers. Figure $3.8 \mathrm{E} 2$ confirms that $\mathrm{H} 160.3$ cells secrete monomers and hemimers and they consist entirely of Hc and Lc. L55 cells used as control was different in many aspects. The biggest difference was noted in the soluble secreted fraction. L55 cells secrete only polymers which are composed of $\mathrm{Hc}$ and $\mathrm{Lc}$. There is a small but detectable amount of hemimer secretion, followed by what appear to be two Lc only fractions. These however are unlikely to be $\mathrm{L}$ chains because an anti- $\mu$ antibody is used to immunoprecipitate the labeled IgM. These could be unknown proteins that cross react mildly with the anti- $\mu$ (Figure 3.8F1 and 3.8F2). As with H160.3 cells, L55 also have polymers, monomers and hemimers intracellularly (Figure 3.8B1 and 3.8B2).

\subsubsection{Kinetics of IgM production and secretion}

To determine the rate of IgM secretion, we performed a pulse chase experiment. Immediately after the pulse at the time point zero, the H160.3 cells showed monomers and hemimers (Figure 3.9A Lane 1). At $2 \mathrm{hrs}$, $4 \mathrm{hrs}$ and 6hrs, polymers are present predominantly with small amount of monomers (Figure 3.9A Lanes 2, 3 and 4). Hemimers are entirely absent inside the cell beginning from the $2 \mathrm{hr}$ time point. When the 
Figure 3.8 2-D Analysis of intracellular and secreted IgM.

Hybridomas H160.3 and L55 were metabolically labeled for $16 \mathrm{hrs}$ with ${ }^{35} \mathrm{~S}$ cysteine and methionine. Cells were lysed and proteins were immunoprecipitated with Goat antimouse $\mu$ antibodies and protein A-Sepharose. Cell lysates prepared with (A and B) or without treatment (C and D) with a cross linker, DSP, were analyzed. Proteins were separated in the first dimension on non-reducing $8 \%$ SDS-polyacrylamide gels. The lanes were cut and placed horizontally across a large well on 10\% SDS-polyacrylamide gels and run under reducing conditions for the second dimension. The major bands in H160.3 cell lysates (A1) under non-reducing conditions are polymer intracellular IgM, and bands at $200 \mathrm{kDa}, 100 \mathrm{kDa}$ and approximately $75 \mathrm{kDa}$. The polymers, and the material at 200 $\mathrm{kDa}$ and $100 \mathrm{kDa}$ contain the $\mu \mathrm{Hc}$ and $\kappa \mathrm{Lc}$ in $\mathrm{H} 160.3$ cells (A2). Intracellular IgM in L55 cells (B1) is similarly composed of $\mu \mathrm{Hc}$ and its $\lambda x \mathrm{Lc}$ (B2). In both cell types the $75 \mathrm{kDa}$ band represents the free intracellular $\mu \mathrm{Hc}$. H160.3 cell lysates (C2) treated with DSP show bands above and below the $\mu \mathrm{Hc}$ in the polymer fraction. These bands might contain scaffold proteins (like ERGIC-53 and /or ERp44) that associate with IgM for polymerization and transport through the intracellular compartments. The smaller $200 \mathrm{kDa}$ band, the $100 \mathrm{kDa}$ band and the $75 \mathrm{kDa}$ bands do not contain additional proteins even after chemical cross linker treatment. L55 cell lysates (D2) under reducing conditions show the same additional bands above and below the polymer fraction as H160.3 cell lysates. Analysis of secreted IgM by H160.3 cells under reducing conditions (E2) shows that the IgM they secrete is entirely made up of $\mu$ and $\kappa$ even though polymers are not secreted. The polymers secreted by L55 cells (F1) resolve into $\mu$ and $\lambda$ under reducing conditions (F2). 

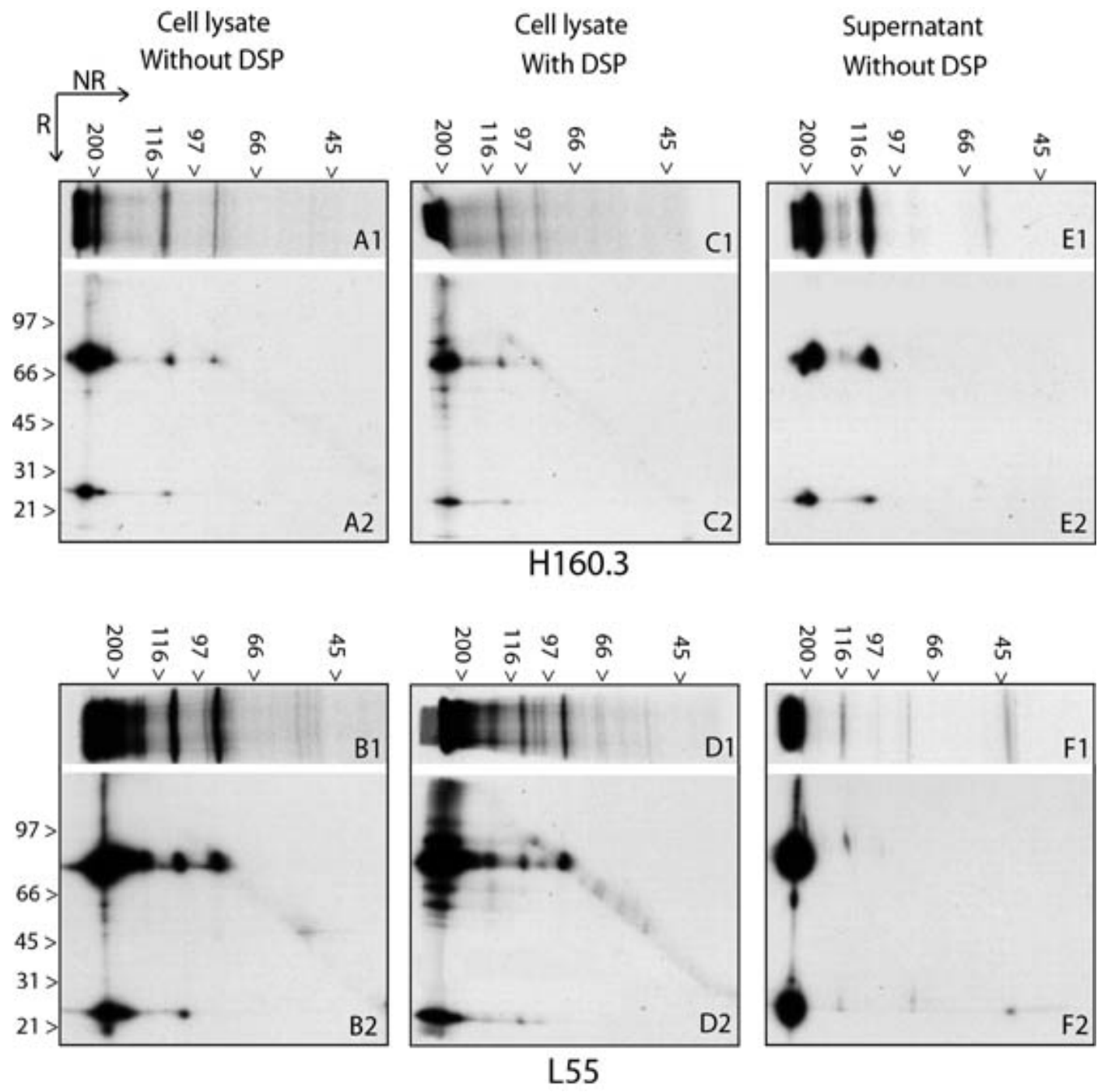
corresponding secreted fractions are examined, it can be seen that the H160.3 cells secrete Ig in the form of monomers and hemimers predominantly. There is also a small amount of polymers present. At $2 \mathrm{hrs}$, the monomers are the main form that is secreted while there is a faint band corresponding to hemimers (Figure 3.9B Lane 2). The amount of hemimers being secreted increases at $4 \mathrm{hrs}$ and the amount of monomers remains the same as at $2 \mathrm{hrs}$ (Figure 3.9B Lane 3). After 6hrs of chase, the amount of hemimers secreted is comparable to that of the monomers (Figure 3.9B Lane 4). The cells begin to extrude spherons only after $4 \mathrm{hrs}$ and they migrate as polymers (Figure 3.9C Lanes 3 and 4).

At the $0 \mathrm{hr}$ time point, the L55 cells showed polymers in addition to monomers and hemimers (Figure 3.9D Lane 1) suggesting that the Ig assembles into polymers as the $\mathrm{Hc}$ and the Lc are synthesized. In the L55 cells, the $80 \mathrm{kDa}$ band likely corresponds to free intracellular Hc as also seen in the 2D gel (Figure 3.8 B1 and B2). Polymers were recovered starting at $2 \mathrm{hrs}$ and are present at all time points. Monomers are present at 2 and 4hrs (Figure 3.9D Lanes 2 and 3). Hemimers exist in small amounts inside the cells up to $4 \mathrm{hrs}$. At 6hrs, all the intracellular IgM is present as polymers (Figure 3.9D Lane 4). L55 secrete polymers predominantly and trace amounts of monomers and hemimers are present (Figure 3.9E Lanes 2-4). L55 do not extrude spherons (Figure 3.9F Lanes 1- 4).

Under reducing conditions, $\mathrm{IgM}$ is visualized as $\mathrm{H}$ and $\mathrm{L}$ chains only. There is a clear difference between H160.3 and L55, in the rate of decrease of intracellular IgM levels beginning at $2 \mathrm{hrs}$. Intracellular IgM levels decrease steadily in L55 cells (Figure 3.9G Lanes 1-4) because it is being secreted normally (Figure 3.9K Lanes 2-4). In contrast, the intracellular IgM level in the H160.3 cells though decreases with time, is not drastic. This indicates that a certain amount of newly synthesized IgM in H160.3 gets retained intracellularly (Figure 3.9J Lanes 1-4) while the secreted IgM fractions increase only slightly at each time point (Figure 3.9H Lanes 2-4).

\subsubsection{Hybridomas differ in glycosylation patterns}

Cell lysates, supernatant and spherons from the three hybridomas were analyzed for the glycosylation patterns. The immunoprecipitated fractions were treated with either Endo $\mathrm{H}$ or PNGase F and compared to untreated IgM fractions. The Hc fraction of 38c Lc expressing hybridomas, when untreated, runs between 97 and $66 \mathrm{kDa}$ which is about the right size for a glycosylated Hc. The cell lysates when treated with Endo H clearly run as two bands one that is resistant to the enzyme and the other that is sensitive (Figure 3.10 I, Lane A/E). Endo H resistance is acquired when the glycoprotein acquires additional and more complex sugar moieties in the cis-Golgi. An Endo H resistant fractionimplies that the protein has exited the ER and is present in the medial or transGolgi, where the high mannose sugars have already been trimmed by the glycosidase in the Golgi (273). Therefore the Endo H resistant fraction suggests that IgM transitions through the Golgi. The Endo H sensitive fraction inside the cells indicates that there is IgM present inside the cell in the ER other than the Golgi accumulated fraction. There is 
Figure 3.9 Pulse-chase analysis of IgM synthesis.

Hybridomas H160.3 and L55 cells were depleted of cysteine and methionine for 40 minutes, then pulsed with ${ }^{35} \mathrm{~S}$ labeled Met/cys for 15 minutes and chased for the indicated times in complete media. The $0 \mathrm{hr}$ time point was taken immediately after the pulse. At each time point, cells (A,D, G and J), media (B,E, H and K) and spherons (C,F, I and L) were prepared and analyzed. Labeled $\mu$ was immunoprecipitated and analyzed by nonreducing and reducing SDS-PAGE as described in Fig 7. In H160.3 cells, the label gets incorporated mostly into monomers and hemimers (Lane 1) after 15 minutes. The entire label becomes incorporated into material migrating as polymers at $2 \mathrm{hrs}$ up to $6 \mathrm{hrs}$ (Lanes 2 - 4). H160.3 cells secrete three forms of IgM - polymers, monomers and hemimers (Lanes 5 - 8). The secreted polymer fraction is very small but it increases slightly from $2 \mathrm{hrs}$ to $6 \mathrm{hrs}$. The monomer fraction remains the same but there is a considerable increase in hemimer secretion from $2 \mathrm{hrs}$ to $6 \mathrm{hrs}$. Increase in hemimer secretion from 2 to $6 \mathrm{hrs}$ is improbable because corresponding intracellular stores are absent. Therefore, the hemimers observed here could be the result of small scale disintegration of monomers or spherons. The labeled IgM begins to appear as spherons in H160.3 cells beginning only at $4 \mathrm{hrs}$ and the spherons appear to decline slightly at $6 \mathrm{hrs}$ (Lanes 11 and 12). In L55 cells, the label gets incorporated into polymers, monomers and hemimers (Lane 13). Hemimers start declining at $2 \mathrm{hrs}$ and $4 \mathrm{hrs}$ but monomers are present up to $4 \mathrm{hrs}$ (Lanes 14 and 15). After $6 \mathrm{hrs}$ of chase, monomers and hemimers are absent completely (Lane 16). L55 cells secrete IgM entirely in the form of polymers (Lanes 18 - 20) and do not extrude spherons (Lanes 21 -24). Intracellular $\mu$ levels decline beginning at $2 \mathrm{hrs}$ (Lanes 25 -28). Under reducing conditions the secreted $\mu$ levels appear to increase with time (Lanes 30 -32). Intracellular $\mu$ levels in L55 decline after chase (Lanes 37 -40) with a corresponding increase in secreted IgM (Lanes 42 -44). 

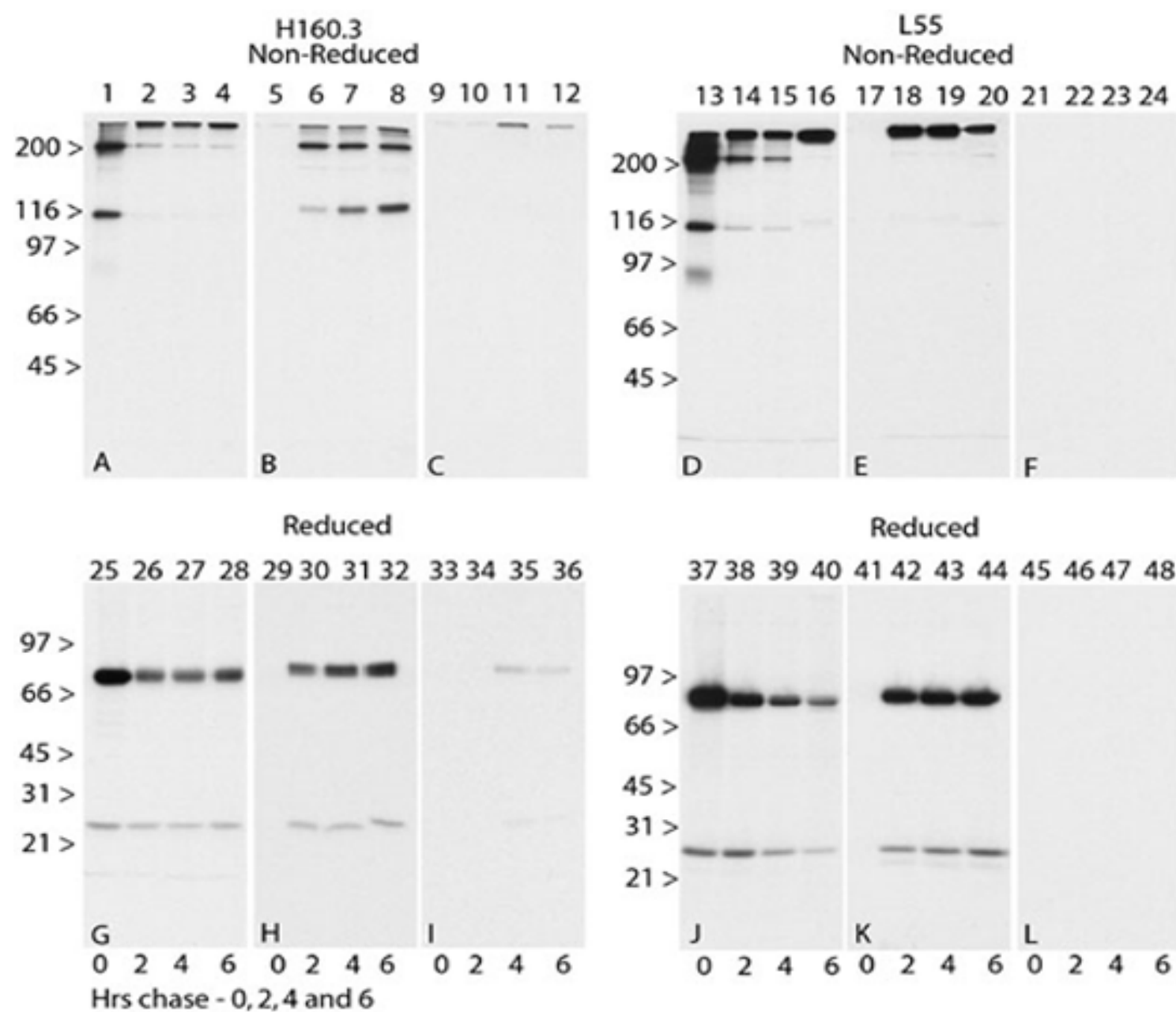

$37383940 \quad 4142 \quad 4344 \quad 45 \quad 4647 \quad 48$

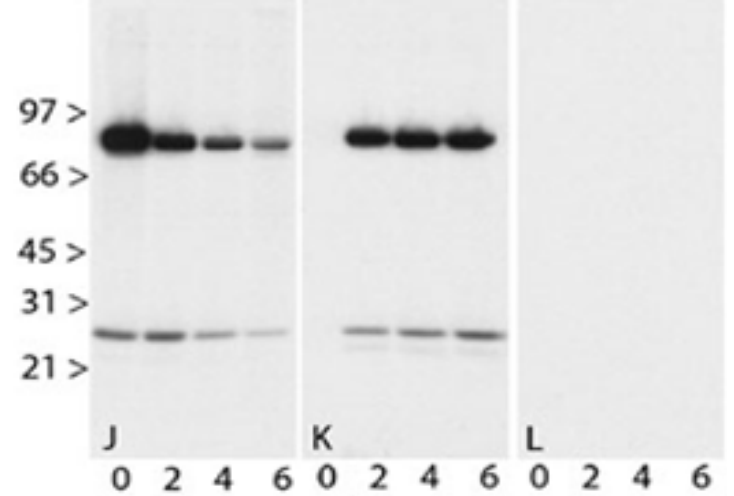


an Endo $\mathrm{H}$ sensitive IgM fraction also that migrates below the resistant fraction. This could represent the IgM in the ER or possibly that in the cis-Golgi since glycans with free terminal mannose residues could be present in proteins at both these locations. In L55 cells, strikingly there is no intracellular Endo H resistant fraction (Figure 3.10 II, Lane $\mathrm{D} / \mathrm{E}$ ). There are two bands but both are sensitive to Endo $\mathrm{H}$ implying that whatever is present in the cell is in the ER and that there is no accumulation of Ig in the Golgi. The presence of two bands can be due to the difference in the processing of sugar moieties incorporated during glycosylation of the Hc in the ER. The band corresponding in size to the $\mathrm{Hc}$ is most likely the chaperone BiP because its size is about $78 \mathrm{kDa}$ similar to that of the Hc (Figure 3.10 II Lane D/E) (272).

In both H160.3 and L55, the secreted IgM is Endo H resistant (Figure 3.10 I, and 3.10 II Lanes B/E, and E/E). This shows that the Ig passes through the Golgi before being secreted. IgM monomers and hemimers also presumably pass through the Golgi because they are Endo H resistant. The spherons are also Endo H resistant (Figure 3.10 I Lane $\mathrm{C} / \mathrm{E}$ ). This shows that though abnormal forms of Ig are secreted, all the secreted fractions pass through the Golgi and acquire complex carbohydrates. PNGase F removes all Nlinked carbohydrate side chains be they simple or complex (274). Therefore all fractions treated with PNGase F run faster compared to the untreated and Endo H treated fractions. The light chains are unaffected by glycosidases since they do not get glycosylated. Therefore the Lc in all treatments and all fractions run at the same level.

The cell lysates were treated with Endo $\mathrm{H}$ and analyzed under reducing conditions to determine at what point the IgM leaves the ER to the Golgi and when the secreted IgM passes from the Golgi to the cell's exterior. The intracellular IgM in the $\mathrm{VH} 56 \mathrm{R} / 76 \mathrm{R}+\mathrm{V} \kappa 38 \mathrm{c}$ cells at $0 \mathrm{hrs}$ is completely sensitive to Endo H (Figure $3.10 \mathrm{III}$, Lane1). Endo H resistant fractions start showing up at $2 \mathrm{hrs}$ (Figure 3.10 III, Lanes 2-4). This indicates that a part of the intracellular IgM that was present in the ER has moved to the Golgi and has had old carbohydrate sugars trimmed and got new sugars added by Golgi glycosidases. Intracellular IgM in L55 cells is entirely sensitive to Endo H at all time points (Figure $3.10 \mathrm{IV}$, Lanes 1-4) indicating that assembled polymers of IgM are not retained in the Golgi at any point even though they clearly pass through it.

\subsection{Discussion}

Our examination of autoreactive B cells that fail to edit their Ig receptors and instead escape central tolerance has uncovered a particularly dangerous class of autoreactive $\mathrm{B}$ cells. These cells produce a $\mathrm{H} / \mathrm{L}$ chain pair that is multireactive, associates into an IgM antibody that accumulates in the Golgi, and arrives at the cell surface in the form of IgM clusters (256). Here, we describe the release of the IgM clusters from the cell surface and the characteristics of such clusters. In vivo, this type of $\mathrm{B}$ cell is enriched in the marginal zone of the spleen, a site that harbors B cells that can be directly activated by antigen. 
Figure $3.10 \mathrm{IgM}$ fractions after glycosidase treatment.

Metabolic labeling, collection of various factions, and immunoprecipitation of $\mu$ proteins from H160.3 and L55 cells were performed as described in Fig 7. After immunoprecipitation, the IgM fractions were treated with EndoH or PNGase F or no enzyme for $1 \mathrm{hr}$ at $37^{\circ} \mathrm{C}$ and then analyzed under reducing conditions. Cell lysates from H160.3 (A) when treated with Endo H (Lane 2) show an Endo H resistant fraction and an Endo $\mathrm{H}$ sensitive fraction suggesting that there is Golgi accumulation of IgM since Endo $\mathrm{H}$ resistance is acquired in the Golgi. PNGase F treated H160.3 lysates are entirely sensitive to the enzyme (Lane 3). Cell lysates from L55 cells (D) are Endo H sensitive (Lane 11) suggesting that little to no IgM resides in the Golgi. Secreted IgM from H160.3 (B) cells is entirely Endo $\mathrm{H}$ resistant (Lane 5) indicating that even IgM secreted as monomers and hemimers, pass through the Golgi and acquire Endo H resistance. L55 cells (E) secrete IgM polymers that are entirely Endo H resistant (Lane 14). Spherons produced by H160.3 cells (C) are Endo H resistant (Lane 8). Cell lysates prepared from $\mathrm{H} 160.3(\mathrm{G})$ and L55 (H) cells after pulse-chase and immunoprecipitation (As described in Fig 7 and 9) were treated with Endo $\mathrm{H}$ for the indicated times and analyzed by SDSPAGE under reducing conditions. At $0 \mathrm{hrs}$, all the intracellular IgM is Endo $\mathrm{H}$ sensitive (Lane 19), as most newly synthesized IgM is present in the ER. IgM acquires Endo H resistance with time (Lanes 20 - 22), and at $6 \mathrm{hrs}$, most of the intracellular IgM is resistant to EndoH (Lane 22), indicating that IgM accumulates intracellularly in the Golgi of H160.3 cells. In contrast, the intracellular IgM in L55 cells is sensitive to Endo H even after 6 hrs (Lanes 23 - 26). 

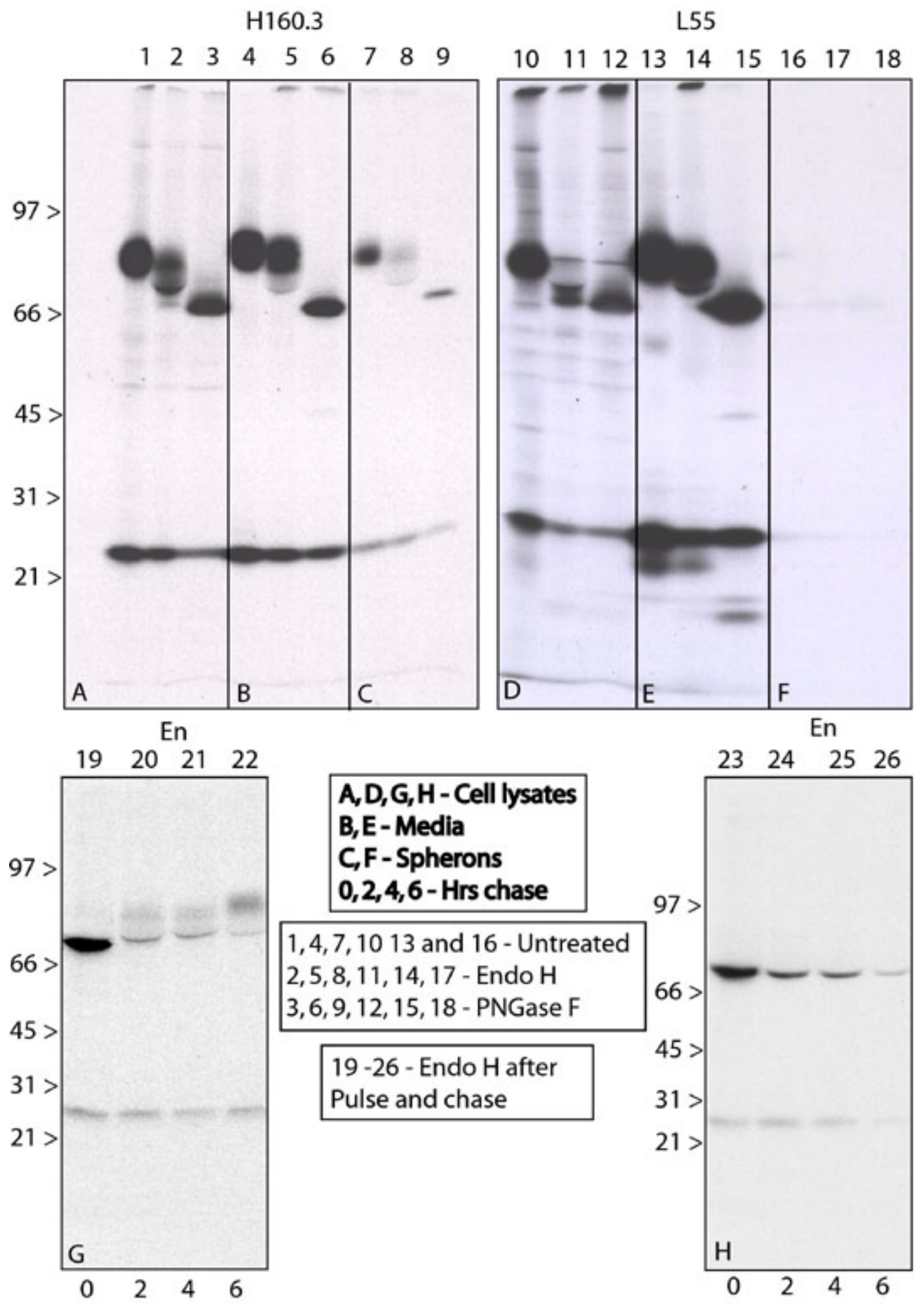
IgM accumulation in the Golgi is fundamentally different from previously known forms of IgM accumulations in the cell. Research on incorrectly folded IgM identified Russell bodies. Russell bodies are Ig that cannot be secreted and yet have escaped intracellular proteasomal degradation and exist within the ER and partitioned off such that the normal secretory pathway is undisturbed (275). Aggresomes, another class of intracellular protein accumulation, arise due to incomplete proteasomal degradation of misfolded proteins. Aggresome formation is microtubule dependent and occurs due to assemblage of smaller aggregates in the cell to the MTOC area (276). Both of these intracellular sequestration mechanisms retain proteins in the cell although in different locations. In contrast, IgM that, as in this case, accumulates in the Golgi, may be fully assembled into IgM pentamers at the time of their release from the cell. The IgM release proceeds by a fundamentally different pathway, as much of the $\operatorname{IgM}$ is packaged in large spherical aggregates, spherons, that settle out of solution, rather than becoming soluble macromolecules. Most (or all) of the soluble IgM that is secreted from the cells is incompletely assembled and is in the form of IgM hemimers and monomers.

The VH56R+Vк38c B cells incorporate the radiolabel mainly into hemimers and monomers and very little polymer formation occurs during the pulse. The hemimers present intracellularly at the start of the chase disappear entirely beginning at two hours and are never observed at the later time points. Folding, glycosylation and disulfide bond formation between Hc and Lc appears to occur without any glitch and the IgM getting

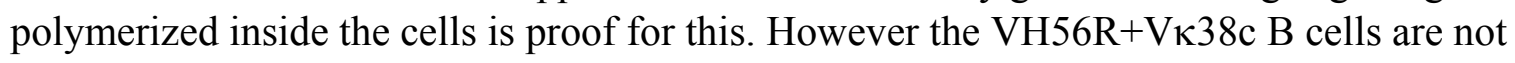
able to sustain the rate of intracellular IgM polymerization required for efficient secretion of pentamers. Indeed the monomers and hemimers begin to appear in the secreted fraction beginning at $2 \mathrm{hrs}$. This may be due to a partial breakdown of the ER quality control in the cells.

Cysteine 575 acts as a retention element within the ER, and undergoes disulfide exchange reactions with resident proteins (73). This process known as thiol-mediated retention (277) is required for efficient degradation of assembly intermediates in resting cells (278). Thiol-mediated retention and intracellular degradation prevent the premature release of IgM assembly intermediates from the ER $(278,279)$. Thiol retention is an extremely efficient process and consequently only trace amounts of monomeric IgM are found in normal serum. In thiol mediated ER retention the interchain disulfide bonds are reduced prior to proteasomal degradation. In the $\mathrm{VH} 56 \mathrm{R}+\mathrm{V} \kappa 38 \mathrm{c}$ cells, there could be a

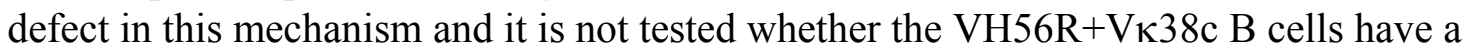
cysteine 575. However, in the VH56R+Vк38c cells the thiol mediated mechanism is likely to be intact due to two observations. First, the IgM accumulation is in the Golgi and not in the ER. Second HL and H2L2 intermediates are not the only components in

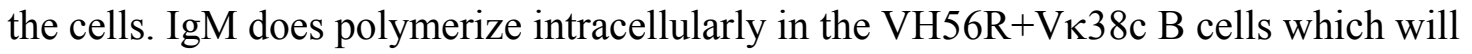
not be the case if thiol mediated retention occurs in the ER. The situation with $\mathrm{VH} 56 \mathrm{R}+\mathrm{V} \kappa 38 \mathrm{c}$ hybridomas is unique because the monomers and hemimers are secreted rather than being retained. This calls for other explanations such as checkpoints in the Golgi by ERp44 (by relocating IgM monomers from the Golgi to the ER for polymerization) going awry. 
It should be pointed out that not all previously reported Ig accumulations remain inside B cells. For example, hybridomas from viable moth-eaten mice contain Russell bodies and yet they can be separated into cells that secrete IgM and others that do not (275). Similarly, the 38C-13 lymphoma cells, which are antigen sensitive resting B lymphocytes, were observed to accumulate IgM do not extrude IgM spherons (SK and MR unpublished). These cells however, secrete incompletely assembled 7S IgM (258). In chronic lymphocytic leukemias of B cells (B-CLLs), impaired glycosylation and misfolded $\mu \mathrm{Hc}$ lead to retention of IgM in the ER and nascent IgM remains unsuitable for secretion (280). Nevertheless, the production of spherons appears to be a surprisingly efficient way to extrude IgM aggregates from the cell.

Our observation is novel to such an extent that it is difficult to precisely assert its relevance in vivo. However, we know that splenocytes from VH56R mice release substantial amounts of spheron-like IgM aggregates after 2 days of culture in the presence of LPS (SK and MR unpublished). Thus, the production and release of IgM spherons is not limited to B lymphocyte-myeloma heterokaryons, but is a potential attribute of primary B cells.

If B cells can produce spherons in vivo, then what might be the fate of such large antibody assemblies? It is unlikely that they would remain in the circulation for any extended period of time. Instead, they may follow the fate of large immune complexes and be cleared through the reticuloendothelial system in the liver (281). However, other sites of spheron capture could include the basement membrane in the kidneys or sites of lymphatic circulation through lymph nodes and the spleen.

Anti-dsDNA antibodies have been eluted from kidneys affected by lupus (282). Eilat et al. showed that some murine anti-dsDNA antibodies were capable of increasing the amount of proteinuria produced by the kidneys, by using an isolated rat kidney perfusion system (283). The potential pathogenicity of human monoclonal anti-dsDNA antibodies was demonstrated in the severe combined immuno-deficient (SCID) mouse mice. Hybridoma cells producing anti-DNA antibodies when implanted intra-peritoneally into these mice, showed the ability to induce significant proteinuria. The proteinuria was associated with the ability of the antibodies to bind to the kidney (284). VH56R+Vк38c hybridomas have the potential to produce similar effects in mice. It remains to be seen if kidneys from VH56R Tg mice show antibody deposition and proteinuria.

Purified anti-dsDNA antibodies from patients with SLE showed cross-reactivity with $\alpha$-actinin and this was most commonly observed in patients with lupus nephritis (285). This argument was strengthened by demonstrating an association between antibody affinity for $\alpha$-actinin and binding to glomeruli in vitro (286) . VH56R+Vк38c $\mathrm{scFv}$ showed affinity to $\alpha$-actinin (SK and MR unpublished) in addition to being a good anti-DNA antibody. Thus VH56R+Vк38c antibody production in vivo could lead to autoimmune disease and the resulting pathological changes.

In considering the possible fates of IgM spherons, we have to consider that they are multireactive. If this multivalency is physiologically relevant, then we may have to 
consider that the spherons may lodge in various tissues based on the specificity of the IgM and thus form nucleation sites for beta amyloid IgM deposits, as are observed with different types of B cell dyscrasias and malignancies. Spherons are relevant in B cell dyscrasias like monoclonal Ig deposition disease (MIDD) (287). Immunoglobulin deposition diseases are usually diagnosed by demonstrating the pathologic deposits by light and electron microscopy. Moreover, the IgM deposits may lead to tissue damage due to the binding and activation of complement.

Cryoglobulins are Ig that precipitate at temperatures below $37^{\circ} \mathrm{C}$. This temperature-sensitive precipitation is usually indicative of a monoclonal plasma cell dyscrasia, a circulating immune complex, or both. Type I cryoglobulins are composed of a monoclonal immunoglobulin, mainly IgG and occasionally IgM or IgA. Clinical manifestations are usually caused by capillary obstruction because of precipitation of the cryoprotein. Capillary obstruction can also occur in the kidney (288). Rare forms of organized monoclonal immunoglobulin deposits related to type I cryoglobulins include glomerulonephritis with organized microtubular monoclonal immunoglobulin deposits (GOMMID) $(289,290)$ a condition associated with various B-cell-derived immunoproliferative disorders, including chronic lymphocytic leukemia (289) and multiple myeloma (291). Antibody secreted by VH56R+Vк38c hybridomas is predominantly monoclonal $\mathrm{IgM}$ and this does not precipitate at temperatures below $37^{\circ} \mathrm{C}$. However spherons produced by the VH56R+Vк38c cells are capable of circulating as immune complexes if produced by cells in vivo. Spherons deposition in kidneys and capillaries could lead to disease similar to that caused by cryoglobulin deposition.

We believe our studies will pave the way for a better understanding of the pathogenesis of Ig deposition diseases. While it is known that these disease entities or their manifestations are all related to increased IgM in the blood serum, the source of the IgM itself is not known. The VH56R B6 mice could serve as an in-vivo model to deduce the mechanisms responsible for Ig aggregation and tissue localization. This along with a better understanding of factors affecting plasma cell activation should help understand disorders associated with abnormal protein deposition. 


\section{CHAPTER 4: GENERAL DISCUSSION}

\subsection{Our search for rules of receptor editing uncovers an exception}

A widely held assumption is that non-autoimmune individuals do not have autoreactive B cells in the periphery. This assumes that B cells with autoreactive receptors are subject to tolerance by clonal deletion, anergy and receptor editing at the immature B cell stage after expression of a fully assembled IgM BCR (292). Receptor editing is the primary mechanism that eliminates autoreactive receptors. Deletion occurs mainly when Ig gene rearrangement is disallowed, constrained or unsuccessful (221). Anergy may result if the BCR signaling that is induced by self antigen is weak, as may be the case for soluble antigens (293). For the different mechanisms, specific antigens, or forms of signaling that initiate B cell regulation have not been fully established.

In this thesis, we tested the prediction that 'binding to autoantigens' induces receptor editing in B cells of non-autoimmune individuals. To this end, we resurrected peripheral $\mathrm{B}$ cell receptors by cloning the $\mathrm{Hc}$ from the $3 \mathrm{H} 9, \mathrm{~V}_{\mathrm{H}} 56 \mathrm{R}$ and the $\mathrm{V}_{\mathrm{H}} 56 \mathrm{R} / 76 \mathrm{R}$ Hc transgenes and providing them with various $\kappa \mathrm{Lc}$. We analyzed their affinity to dsDNA and found that some receptors had high affinity to DNA while others did not bind DNA very well. We delineated a threshold range based on affinity of these recombinant receptors to DNA. Consistent with effective regulation, receptors that bound DNA with high affinity were absent from the periphery while those that did not have much affinity were present. Thus, avid anti-self BCR were absent from the periphery, possibly because of receptor editing. Our observations are in accord with other studies that have shown that receptor editing eliminates a large portion of immature $\mathrm{B}$ cell receptors that bind DNA $(122,124,125,218,294)$.

In our study however, we discovered one conspicuous exception that does not fit the proposed threshold mechanism. The combinations of $\mathrm{V} \kappa 38 \mathrm{c}$ with either VH56R or VH56R/76R bound DNA very well and yet were present in the periphery. Crucial for this exceptional result may be that these receptors had specificity for the Golgi in addition to DNA. Specificity of the VH56R+Vк38c receptors for the Golgi may sustain developing $\mathrm{B}$ cells harboring these receptors and allow them into the periphery. It remains to be seen if our exception represents an isolated case or, alternatively, a generalized mechanism for escape of autoreactive B cells to the lymphoid periphery.

\subsection{Our efforts to identify the mechanism for escape of VH56R+Vк38c cells}

With our colleagues at the University of Chicago and the University of Pennsylvania, we then examined B cells from mice expressing the relevant Hc transgenes. We noticed that surface BCR densities were reduced in the immature B cells (Hardy fraction E) carrying the VH56R anti-DNA Hc. We conceptualized that the $\mathrm{VH} 56 \mathrm{R}+\mathrm{V} \kappa 38 \mathrm{c} \mathrm{B}$ cells might be part of this population and the intracellular BCR in these cells, when moving to the cell surface, may be delayed or impeded due to binding to a 


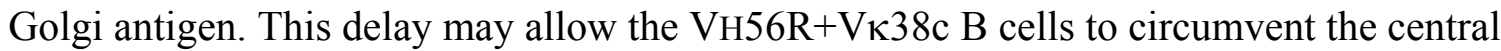
tolerance mechanisms. Receptor retention as in the H160.3 hybridomas may provide a new mechanism that facilitates the escape of autoreactive cells to the periphery. It should be pointed out that the $\mathrm{VH} 56 \mathrm{R}+\mathrm{V} \kappa 38 \mathrm{c}$ BCR in the mature $\mathrm{B}$ cell populations may not be entirely retained intracellularly and likely reaches the cell surface. Other mechanisms, for example physiological UPR may be responsible for this observation. Nevertheless, it is at the immature B cell stage that B cells are tolerized and the reduction or absence of receptor at this stage might be the most important reason for the escape and development of the VH56R+Vк38c B cells. Why the receptor might be reduced/absent at the cell surface only at the immature B cell stage and not in the periphery remains to be investigated.

To understand the mechanism of receptor retention, we asked what could account for the intracellular accumulation of the $\mathrm{VH} 56 \mathrm{R}+\mathrm{V} \kappa 38 \mathrm{c}$ receptor? One, reason might be

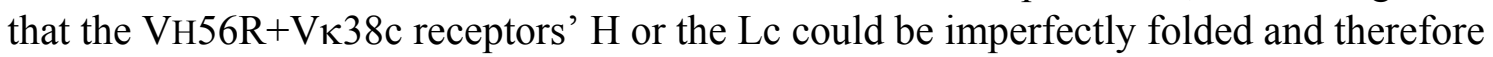
are retained inside the cell by quality control mechanisms. Folding defects (295) or deliberate removal of Hc domains results in the intracellular retention of antibody components in the ER (but not in the Golgi) and the accumulation is associated with degradation of the Ig chains after dislocation from the ER to the proteasome (296). Alternatively, the receptors are properly folded but they bind an antigen in the Golgi. We favor the second possibility because the VH56R+Vк38c antibody co-localizes with Golgi markers in hybridomas indicating that the antibody is retained in the Golgi. Moreover, binding of the recombinant $\mathrm{VH} 56 \mathrm{R}+\mathrm{V} \kappa 38 \mathrm{c}$ receptors to the Golgi in Jurkat cells supports the idea of a Golgi antigen. As far as we can determine, the VH56R+Vк38c cells have no defect in either the Hc or the Lc, nor in their assembly into IgM pentamers, further supporting our reasoning that accumulation is due to the BCR binding to antigen in the Golgi.

We conclude that the crucial B cell tolerance mechanisms, editing, deletion and anergy will work only when B cell receptors reach the cell surface. If, on the other hand, the B cell receptor is restrained intracellularly by some resident antigen, regulation will be ineffective. Therefore deletion, anergy and editing are not universal mechanisms that influence B cell development. Instead, they are influenced by the location of BCR or access to antigens.

\subsection{Determining the location of $\mathrm{VH} 56 \mathrm{R}+\mathrm{V} \kappa 38 \mathrm{c}$ accumulation}

We have used giantin, golgin-95, $\beta$-COP, lectin II and $\alpha$-mannosidase II to resolve the exact location of 38c IgM. Giantin and golgin-95 together with p115 (a peripheral membrane protein localized to the ERGIC and cis-Golgi that binds to both giantin and golgin-95) play important roles in retrograde transport, anti-retrograde transport, cisternal stacking and vesicle tethering at the cis-Golgi (197). Thus giantin and golgin-95 are considered to be cis-Golgi markers. Since VH56R+Vк38c does not colocalize entirely with either giantin or golgin-95 it must be in a compartment that is either before or after cis-Golgi. One compartment that occurs before the cis-Golgi is the 
ERGIC. However, colocalization with $\beta$-COP is also partial, which means ERGIC may not be the location for VH56R+Vк38c. On the other hand lectin II binds to free GlcNAc residues and is considered a Golgi marker because most free GlcNAc are present in the medial-Golgi. Thus the most intense signal from Lectin II comes from the medial-Golgi. A major percentage of lectin II is present inside the hybridomas co-localizes with the $\mathrm{VH} 56 \mathrm{R}+\mathrm{V} \kappa 38 \mathrm{c}$ IgM. $\alpha$-mannosidase II is considered to be a marker for a broader region of the Golgi (297). This is because only two-thirds of the $\alpha$-mannosidase II marker labeling is in the medial-Golgi while the rest is in the cis-Golgi cisternae (298). Our results show that a high percentage of VH56R $+\mathrm{V} \kappa 38 \mathrm{c}$ localizes with that of the $\alpha$ mannosidase II staining. This suggests that $\mathrm{VH} 56 \mathrm{R}+\mathrm{V \kappa} 38 \mathrm{c}$ is distributed predominantly in the medial-Golgi (to the trans-Golgi) compartment while a smaller percentage occurs in the preceding Golgi compartments. Our conclusion is supported by the co-localization of VH56R+Vк38c IgM and golgin-95 in cells treated with nocodazole where intracellular IgM disperses to the same locations as golgin-95.

Epitopes for AGA are located primarily on the cytoplasmic face of the Golgins. How these coiled-coil golgins become autoimmune targets is not known. One reason that might contribute to Giantin being the more common Golgi antigen is that it is a transmembrane protein which can be more stably associated with the Golgi membrane that the other golgins which are only peripheral membrane proteins (299). It was postulated that these Golgi proteins may be recognized by the immune system in disease states involving apoptosis or necrosis along with defective clearance (197). Golgi morphology varied from being swollen to appearing as vesicular structures during apoptosis. However most importantly, none of the golgins could be detected on the surface of apoptotic blebs (299). Thus the scenario where antigen exposure during apoptosis, leading to AGA production, may be unlikely. On the other hand, escape and development of VH56R+Vк38c B cells might offer a potential answer to the development of AGAs. In this case it is possible to envision a situation where the Golgi antigens do not have to be on the cell surface for the immune system to "see" them. The Golgi antigens though are sequestered intracellularly may bind to the VH56R+Vк38c $\mathrm{BCR}$ allowing B cells to escape to the periphery. Once these VH56R+Vк38c B cells get activated by appropriate antigens, the B cells may start producing antibodies that would be detected as AGAs in the serum.

Currently, we do not know what might be the Golgi antigen for the $\mathrm{VH} 56 \mathrm{R}+\mathrm{V \kappa} 38 \mathrm{c}$ receptor. Golgi has a vital role in secretion, lipid biosynthesis, protein modification and sorting. Therefore numerous targets could potentially function as the antigen for the VH56R+Vк38c receptor. For example, glycosidases and glycosyltransferases that aid in the synthesis of N-Glycans could be the targets. $\alpha$ mannosidase II, a transmembrane glycosidase is particularly interesting because it has a large lumenal C-terminal catalytic domain (273) which theoretically can interact with VH56R+Vк38c while the BCR is present intracellularly. Resident proteins that form the Golgi matrix, like golgin-95, grasp 65, and grasp 55 have small lumenal domains (300) and these proteins are less likely to bind to the VH56R+Vк38c antibody. Rather than a protein antigen $\mathrm{VH} 56 \mathrm{R}+\mathrm{V} \kappa 38 \mathrm{c}$ could bind to a carbohydrate that is formed in the Golgi. 


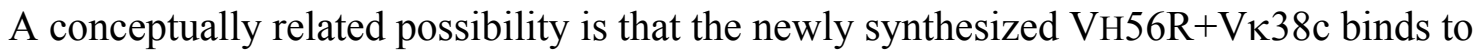
the carbohydrate side chains that are present on the Hc of neighboring $\operatorname{IgM}$ molecules. Each of these possibilities could explain why we see intra-Golgi accumulation in hybridomas.

Glycosaminoglycans (GAGs) are a group of molecules that possibly can bind the $\mathrm{VH} 56 \mathrm{R}+\mathrm{V \kappa} 38 \mathrm{c}$ antibody. Hyaluronic acid is unique among the GAGs because GAGs such as aggrecan are synthesized in the Golgi while the biosynthesis of hyaluronan occurs on the plasma membrane. During the synthesis, the carbohydrate built inside the plasma membrane is pushed out to the extracellular space (301). Hyaluronic acid polymers are very large (with molecular weights of $300 \mathrm{KDa}$ to $2000 \mathrm{KDa}$ ) (302). When the IgM that is synthesized intracellularly, reaches the plasma membrane, it can bind with the hyaluronan to increase in size at the cell membrane and then detach after reaching a certain size. It is possible that not all spherons may interact with hyaluronan at the cell surface and may detach from the cell membrane. This would explain the various sizes of the spherons that settle around the cells.

\subsection{Our proposed mechanism for the production of hemimers and spherons}

The biogenesis of secretory IgM occurs in two sequential but independent steps which are assembly and polymerization. $\mu 2 / \mathrm{L} 2$ assembly occurs in the ER with help from $\mathrm{Hc}$ binding protein $(\mathrm{BiP})$ which acts as a chaperone for the intermediates in assembly. ERp44 and ERGIC-53 play a role in the polymerization of IgM by acting as scaffolds for the formation of a planar complex of monomers (99). ERGIC-53 binds to glycoproteins in the ER and transports them to the Golgi where they are released (303). ERp44 interacts with ERGIC-53 and accumulates in the cis-Golgi (99). To begin with, the assembly of the $\mathrm{VH56R}+\mathrm{V \kappa} 38 \mathrm{c}$ IgM itself may not proceed to completion since we do notice small amounts of monomers even after $6 \mathrm{hrs}$ of chase. This could be due to a partial breakdown during ER quality control that involves ERp44 because it is this protein that is considered to load the IgM monomers onto the scaffold for polymerization. The IgM fraction that does polymerize in the ER is transported to the Golgi by ERGIC-53 after successfully passing the first BiP quality control step. However as IgM starts accumulating in the medial-Golgi it may cause further demands on the scaffold proteins. It has been shown that down regulation of ERp44 and ERGIC-53 reduces IgM polymerization and increases secretion of smaller forms of IgM (99). Thus, as polymerization is hampered, IgM

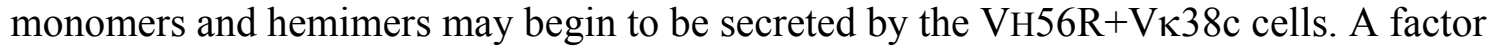
that may contribute to the secretion of hemimers is that they have a reduced avidity to antigen as compared to fully assembled IgM and therefore have an advantage in leaving the Golgi.

The $\mathrm{J}$ chain is a polypeptide chain made by B cells that is required for $\mathrm{IgM}$ pentamer formation. In the absence of $\mathrm{J}$ chain, $\operatorname{IgM}$ is polymerized into hexamers (78, 304). Absence of J chain inhibits the assembly and/or secretion of IgM (305). We can detect $\mathbf{J}$ chain in the hybridomas, and $\operatorname{IgM}$ can polymerize. However, most polymerized IgM is released from the B cells in the form of spherons. Because the spherons have the $\mathrm{J}$ 
chain, at least some of the IgM must be in the form of pentamers. Therefore we conclude that the $\mathrm{VH} 56 \mathrm{R}+\mathrm{V} \kappa 38 \mathrm{c}$ hybridomas are capable of producing complete IgM pentamers.

Formation of spherons is likely linked directly to the location of intracellular IgM accumulation. Defects in $\mu \mathrm{Hc}$ folding or absence of domains that bind to the Lc/chaperones, result in IgM accumulation in the ER as Russell bodies. The Russell bodies are sequestered such that the normal secretory function is unperturbed. However the Russell bodies are not secreted or at least not reported yet. Spherons produced by the $\mathrm{VH} 56 \mathrm{R}+\mathrm{V} \kappa 38 \mathrm{c}$ hybridomas represent the $\mathrm{IgM}$ fraction that was probably retained in the Golgi (rather than in the ER). The idea is definitely conceivable that the location of IgM accumulation either allows the secretion or retention of IgM. The characteristic round shape of the spherons could be due to the the protein assuming the most thermodynamically favorable conformation or assume their shape after possibly binding to some antigen (eg. GAGs at the plasma membrane).

\subsection{Considering the fate of $\mathrm{VH} 56 \mathrm{R}+\mathrm{V} \kappa 38 \mathrm{c} \mathrm{B}$ cells in vivo}

We have determined that the majority of $\mathrm{VH} 56 \mathrm{R}+\mathrm{V \kappa} 38 \mathrm{c} \mathrm{B}$ cells reside in the $\mathrm{MZ}$ of the spleen. MZ B cells react quickly in a $T$ cell independent manner to antigens from blood borne pathogens and begin to secrete IgM. Thus, VH56R+Vк38c B cells could be activated by a suitable antigen associated with a bacterial infection. Once activated, the cells may begin to synthesize large amounts of IgM leading to IgM accumulation inside the cells. The accumulation in turn might lead to the production of spherons. In fact, splenocytes from 56R Tg mouse stimulated with LPS show spherons being produced. Spherons are similar to the immune complex deposits in the glomerulus of SLE patients. Spherons may, because of their size and surface characteristics lodge at the pores of the basement membrane and attract complement deposition. Such events are seen in glomerulonephritis. Thus VH56R+Vк38c B cells or similar B cells that have escaped central tolerance mechanisms could be responsible for the secretion of autoantibodies and immune complexes that cause pathogenesis in SLE.

\subsection{Future directions}

One novel aspect of this thesis is the identification of a new mechanism for autoreactive $\mathrm{B}$ cells to escape the BM regulation. All the evidence presented thus far suggests that BCR retention in the Golgi may be responsible for the escape of autoreactive B cells. Our proposal that the intracellular antibody retention is responsible for autoreactive $\mathrm{B}$ cell escape can be more conclusively proved once an anti-idiotype reagent to the $\mathrm{VH} 56 \mathrm{R}+\mathrm{V} \kappa 38 \mathrm{c}$ antibody becomes available. With this reagent one could analyze the intracellular and cell surface $\mathrm{VH} 56 \mathrm{R}+\mathrm{V \kappa} 38 \mathrm{c}$ antibody expression levels in mice. Low surface expression levels and high intracellular levels in immature B cells could provide proof that antibody retention might be a mechanism for autoreactive $\mathrm{B}$ cell escape. Anti-idiotype antibodies against a B cell lymphoma antibody, 38C-13, have been produced by using idiotype conjugated to keyhole limpet hemocyanin $(306,307)$. Anti- 
idiotypes and bi-specific antibodies have been generated to be used as treatments for lymphomas $(308,309)$. Bi-specific antibodies that bind to an adhesion receptor and tumor specific antigen at the same time are effective in blocking tumor metastasis. A 38c hybridoma secreting large amounts of $\operatorname{IgM}$ can be grown in the peritoneal cavity of a pristane primed mouse to generate the idiotype immunogen (Id). This can be conjugated with keyhole limpet hemocyanin and injected in mice along with complete Freund's adjuvant to generate the $38 \mathrm{c}$ anti-idiotype antibodies (306).

If the VH56R+Vк38c antibody retention in the Golgi is entirely due to the specificity of the antibody, then the Golgi specificity of this antibody can be eliminated by mutations (induced by addition of mutagens in culture). Production of spherons and intracellular accumulation is likely linked. Therefore, VH56R+Vк38c B cells can be sub cloned and the resulting colonies can be tested by light microscopy for spheron production. IgM production in each of these colonies can be tested by anti-IgM ELISA. Colonies that are negative for spheron production but positive for IgM production can be selected and the cells from such colonies can be checked by confocal microscopy for Golgi retention. IgM from such cells can be tested for anti-DNA activity. VH56R+Vк38c antibody that loses Golgi specificity but retains DNA binding will be interesting because now this antibody is like any other anti-DNA antibody. If a transgenic mouse expressing

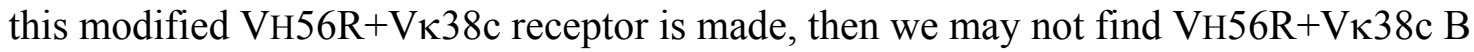
cells in the periphery. This is because without the Golgi specificity, the receptors will reach the cell surface and will be subjected to the normal regulatory mechanisms in the $\mathrm{BM}$. Instead of relying on mutations in hybridomas, $\mathrm{VH} 56 \mathrm{R}+\mathrm{V} \kappa 38 \mathrm{c} \mathrm{scFv}$ with mutated Lc residues can be cloned and can be tested for antigen binding in Jurkat cells. $\mathrm{VH} 56 \mathrm{R}+\mathrm{V} \kappa 38 \mathrm{c} \mathrm{scFv}$ that do not show Golgi binding can be further characterized and used to create newer transgenic mice models.

A related experiment would be to prove Golgi retention occurs in primary $\mathrm{VH} 56 \mathrm{R}+\mathrm{V \kappa} 38 \mathrm{c} \mathrm{B}$ cells and that retention is due to receptor specificity. One way to do this would be to use retroviral transfection in cells that are 56R $\mathrm{Hc}^{+}$to introduce Vк38c and show Golgi localization of the receptor. Retro viral vectors can be constructed by inserting full length cDNAs of Vk38c or mutated VK38c Lc into a backbone that has a

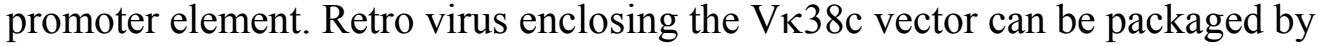
transfecting the vectors into a packaging cell line. Virus stocks containing the vectors can be prepared by collecting the media. 56 $\mathrm{R} \mathrm{Hc}^{+}$cells can be infected with the retro virus and the 56R $\mathrm{Hc}^{+}$cells can be analyzed after about a week of infection. The 38C-13 lymphomas have only the VK38c Lc and yet show the intracellular accumulation. Thus the intracellular accumulation could solely be due to the VК38c Lc and to test that, the Vк38c retroviral vector can be transfected in splenocytes to test whether this would recreate the antibody accumulation. VH56R or VH56R/76R Tg mice can be activated generically and their splenic B cells can be checked to see whether increased intracellular $\operatorname{IgM}$ is a common phenomenon.

VH56R+Vк38c B cells secreting IgM polymers in the form of spherons, has been the most unusual finding in this study. Live cell imaging could be used to observe features of the formation and growth of the spherons inside the cells. In addition, we 
could see how they move to the cell surface and how they detach. Alternatively, GFP linked Lc constructs can be transfected in 56 $\mathrm{R} \mathrm{Hc}^{+}$cells to track the formation and movement of spherons. Primary B cells are capable of producing spherons in the periphery. $\mathrm{VH} 56 \mathrm{R}+\mathrm{V} \kappa 38 \mathrm{c} \mathrm{B}$ cells from the $\mathrm{FO}$ or the $\mathrm{MZ}$ could be sorted using the antiidiotype antibody and placed in culture to ask which group is capable of producing spherons. Moreover, the 38c antibody levels reportedly increase in older transgenic mice (NLP unpublished observation) and the antibodies detected in serum of older mice could include all forms of $\mathrm{VH} 56 \mathrm{R}+\mathrm{V} \kappa 38 \mathrm{c}$. Nevertheless in older mice, the spherons could get deposited in the kidneys instead of circulating in the blood stream. Thus a histochemical examination of the kidneys could be performed to look for antibody deposits. To get a clearer idea about possible deposition sites for the spherons, labeled spherons can be injected to detect the sites where they get localized such as the joints or kidneys etc by using a technique called the "Micro positron emission tomography" (PET). PET determines the three-dimensional distribution of a radiolabeled agent in vivo. Mice could be injected with radiolabeled spherons via the lateral tail vein or via the external jugular vein after anesthetizing them. Data is collected by the microPET-R 4 scanner. This scanner is a small-animal PET scanner and contains detectors capable of axial and transaxial resolution. Images are reconstructed into two-dimensional projections using specialized software (310).

We have proposed that IgM secreted as monomers and hemimers by the VH56R + V 38 c B cells could be due to the scaffold proteins (ERGIC-53 and ERp44) being constrained due to demands imposed by accumulated IgM in the Golgi. Unpolymerized $\mu_{2} \mathrm{~L}_{2}$ monomers if present in the Golgi are bound by ERp44 to be brought back to the ER for polymerization and ERGIC-53 transports the IgM pentamers to the Golgi (99). If Golgi has accumulated IgM then ERGIC-53 might be unable to unload the IgM pentamer and be trapped there creating a depletion in the ER. This could cause more monomers to accumulate in the ER which could soak up the ERp44 in the ER. Now if a monomer leaves the ER unpolymerized into the Golgi, it cannot be brought back to the ER for polymerization because all the ERp44 are occupied. Hence the monomers and hemimers likely get secreted in the absence of a quality control step. We could use confocal microscopy to test the location of ERGIC-53 and ERp44 in H160.3 hybridomas to test the above hypothesis. ERGIC-53 might be concentrated more in the Golgi while ERp44 may stain more in the ER if our hypothesis holds true.

The most difficult and challenging task will be the identification of the target antigen of the 38c antibody in the Golgi. Our data suggests that there is an antigen in the Golgi, but this has not been able to be recovered along with the IgM. At the very least, it must be investigated whether the antigen is protein, lipid or carbohydrate. Our data shows that the IgM accumulates in the medial-Golgi, and therefore proteins present in the medial Golgi can be tentatively selected and placed on a chip. A protein microarray could be used to test the $38 \mathrm{c} \mathrm{IgM}$. However it must be distinguished as to which fraction of the $38 \mathrm{c} \mathrm{IgM} \mathrm{binds} \mathrm{to} \mathrm{which} \mathrm{of} \mathrm{the} \mathrm{medial-Golgi} \mathrm{antigens.} \mathrm{Since} \mathrm{38c} \mathrm{hybridoma} \mathrm{supernatants}$ are polyreactive it is possible that $38 \mathrm{c}$ antibodies in hybridoma supernatant will show binding to many or most antigens on the chip. Therefore it is necessary to separate the different IgM fractions by size exclusion (various fractions will have different Svedberg 
units) after ultracentrifugation and then test them for antigen binding. Lipids and carbohydrates can be tested by ELISA and as before may have to be selected tentatively.

Overall, data documented in this thesis, likely forms the basis of an important new mechanism for escape of autoreactive B cells to the periphery. It remains to be seen if this phenomenon is applicable in both healthy and autoimmune prone individuals. IgM secretion has been studied extensively and the consequences due to defects or variations in components of the IgM molecule have been well documented. However, as far as we can determine, it is the first time spherons have been described and in such detail with extensive characterization. The discovery of spherons has the potential to alter traditional views about immune complex deposition in disease and offer new insights into the origins of immune complexes during pathogenesis of autoimmune disorders. 


\section{LIST OF REFERENCES}

1. Nunez, C., N. Nishimoto, G.L. Gartland, L.G. Billips, P.D. Burrows, H. Kubagawa, and M.D. Cooper. 1996. B cells are generated throughout life in humans. J Immunol 156:866-872.

2. Opstelten, D., and D.G. Osmond. 1983. Pre-B cells in mouse bone marrow: immunofluorescence stathmokinetic studies of the proliferation of cytoplasmic mu-chain-bearing cells in normal mice. J Immunol 131:2635-2640.

3. Jacobsen, K., J. Kravitz, P.W. Kincade, and D.G. Osmond. 1996. Adhesion receptors on bone marrow stromal cells: in vivo expression of vascular cell adhesion molecule-1 by reticular cells and sinusoidal endothelium in normal and gamma-irradiated mice. Blood 87:73-82.

4. Whitlock, C.A., D. Robertson, and O.N. Witte. 1984. Murine B cell lymphopoiesis in long term culture. J Immunol Methods 67:353-369.

5. Ye, M., and T. Graf. 2007. Early decisions in lymphoid development. Curr Opin Immunol 19:123-128.

6. Kikuchi, K., A.Y. Lai, C.L. Hsu, and M. Kondo. 2005. IL-7 receptor signaling is necessary for stage transition in adult B cell development through up-regulation of EBF. J Exp Med 201:1197-1203.

7. Hermiston, M.L., Z. Xu, and A. Weiss. 2003. CD45: a critical regulator of signaling thresholds in immune cells. Annu Rev Immunol 21:107-137.

8. Sanchez, M., Z. Misulovin, A.L. Burkhardt, S. Mahajan, T. Costa, R. Franke, J.B. Bolen, and M. Nussenzweig. 1993. Signal transduction by immunoglobulin is mediated through Ig alpha and Ig beta. $J$ Exp Med 178:1049-1055.

9. DeFranco, A.L., P.R. Mittelstadt, J.H. Blum, T.L. Stevens, D.A. Law, V.W. Chan, S.P. Foy, S.K. Datta, and L. Matsuuchi. 1994. Mechanism of B cell antigen receptor function: transmembrane signaling and triggering of apoptosis. Adv Exp Med Biol 365:9-22.

10. Hayakawa, K., R.R. Hardy, A.M. Stall, and L.A. Herzenberg. 1986. Immunoglobulin-bearing B cells reconstitute and maintain the murine Ly-1 B cell lineage. Eur J Immunol 16:1313-1316.

11. Chen, X., F. Martin, K.A. Forbush, R.M. Perlmutter, and J.F. Kearney. 1997. Evidence for selection of a population of multi-reactive B cells into the splenic marginal zone. Int Immunol 9:27-41.

12. Mahnke, K., M. Guo, S. Lee, H. Sepulveda, S.L. Swain, M. Nussenzweig, and R.M. Steinman. 2000. The dendritic cell receptor for endocytosis, DEC-205, can recycle and enhance antigen presentation via major histocompatibility complex class II-positive lysosomal compartments. J Cell Biol 151:673-684.

13. Turley, S.J., K. Inaba, W.S. Garrett, M. Ebersold, J. Unternaehrer, R.M. Steinman, and I. Mellman. 2000. Transport of peptide-MHC class II complexes in developing dendritic cells. Science 288:522-527.

14. Zheng, B., S. Han, and G. Kelsoe. 1996. T helper cells in murine germinal centers are antigen-specific emigrants that downregulate Thy-1. J Exp Med 184:10831091. 
15. Batista, F.D., D. Iber, and M.S. Neuberger. 2001. B cells acquire antigen from target cells after synapse formation. Nature 411:489-494.

16. Berek, C., A. Berger, and M. Apel. 1991. Maturation of the immune response in germinal centers. Cell 67:1121-1129.

17. McHeyzer-Williams, M.G., and R. Ahmed. 1999. B cell memory and the longlived plasma cell. Curr Opin Immunol 11:172-179.

18. Allen, C.D., T. Okada, H.L. Tang, and J.G. Cyster. 2007. Imaging of germinal center selection events during affinity maturation. Science 315:528-531.

19. Banchereau, J., F. Bazan, D. Blanchard, F. Briere, J.P. Galizzi, C. van Kooten, Y.J. Liu, F. Rousset, and S. Saeland. 1994. The CD40 antigen and its ligand. Annu Rev Immunol 12:881-922.

20. Armitage, R.J., W.C. Fanslow, L. Strockbine, T.A. Sato, K.N. Clifford, B.M. Macduff, D.M. Anderson, S.D. Gimpel, T. Davis-Smith, C.R. Maliszewski, and et al. 1992. Molecular and biological characterization of a murine ligand for CD40. Nature 357:80-82.

21. Kuhn, R., K. Rajewsky, and W. Muller. 1991. Generation and analysis of interleukin-4 deficient mice. Science 254:707-710.

22. Honjo, T., K. Kinoshita, and M. Muramatsu. 2002. Molecular mechanism of class switch recombination: linkage with somatic hypermutation. Annu Rev Immunol 20:165-196.

23. Okazaki, I., K. Yoshikawa, K. Kinoshita, M. Muramatsu, H. Nagaoka, and T. Honjo. 2003. Activation-induced cytidine deaminase links class switch recombination and somatic hypermutation. Ann N Y Acad Sci 987:1-8.

24. Rada, C., G.T. Williams, H. Nilsen, D.E. Barnes, T. Lindahl, and M.S. Neuberger. 2002. Immunoglobulin isotype switching is inhibited and somatic hypermutation perturbed in UNG-deficient mice. Curr Biol 12:1748-1755.

25. Casellas, R., A. Nussenzweig, R. Wuerffel, R. Pelanda, A. Reichlin, H. Suh, X.F. Qin, E. Besmer, A. Kenter, K. Rajewsky, and M.C. Nussenzweig. 1998. Ku80 is required for immunoglobulin isotype switching. Embo J 17:2404-2411.

26. Rogozin, I.B., and M. Diaz. 2004. Cutting edge: DGYW/WRCH is a better predictor of mutability at $\mathrm{G}: \mathrm{C}$ bases in Ig hypermutation than the widely accepted RGYW/WRCY motif and probably reflects a two-step activation-induced cytidine deaminase-triggered process. J Immunol 172:3382-3384.

27. Neuberger, M.S., and C. Milstein. 1995. Somatic hypermutation. Curr Opin Immunol 7:248-254.

28. Hardy, R.R., and K. Hayakawa. 1995. B-lineage differentiation stages resolved by multiparameter flow cytometry. Ann N Y Acad Sci 764:19-24.

29. Hardy, R.R., C.E. Carmack, S.A. Shinton, J.D. Kemp, and K. Hayakawa. 1991. Resolution and characterization of pro-B and pre-pro-B cell stages in normal mouse bone marrow. $J$ Exp Med 173:1213-1225.

30. Oettinger, M.A., D.G. Schatz, C. Gorka, and D. Baltimore. 1990. RAG-1 and RAG-2, adjacent genes that synergistically activate V(D)J recombination. Science 248:1517-1523.

31. Meek, K. 1990. Analysis of junctional diversity during B lymphocyte development. Science 250:820-823. 
32. Schable, K.F., R. Thiebe, A. Bensch, J. Brensing-Kuppers, V. Heim, T. Kirschbaum, R. Lamm, M. Ohnrich, S. Pourrajabi, F. Roschenthaler, J. Schwendinger, D. Wichelhaus, I. Zocher, and H.G. Zachau. 1999. Characteristics of the immunoglobulin Vkappa genes, pseudogenes, relics and orphons in the mouse genome. Eur J Immunol 29:2082-2086.

33. Thiebe, R., K.F. Schable, A. Bensch, J. Brensing-Kuppers, V. Heim, T. Kirschbaum, H. Mitlohner, M. Ohnrich, S. Pourrajabi, F. Roschenthaler, J. Schwendinger, D. Wichelhaus, I. Zocher, and H.G. Zachau. 1999. The variable genes and gene families of the mouse immunoglobulin kappa locus. Eur $J$ Immunol 29:2072-2081.

34. Tonegawa, S. 1983. Somatic generation of antibody diversity. Nature 302:575581.

35. Kerr, W.G., M.D. Cooper, L. Feng, P.D. Burrows, and L.M. Hendershot. 1989. Mu heavy chains can associate with a pseudo-light chain complex (psi L) in human pre-B cell lines. Int Immunol 1:355-361.

36. Tsubata, T., and M. Reth. 1990. The products of pre-B cell-specific genes (lambda 5 and VpreB) and the immunoglobulin mu chain form a complex that is transported onto the cell surface. $J$ Exp Med 172:973-976.

37. Hombach, J., L. Leclercq, A. Radbruch, K. Rajewsky, and M. Reth. 1988. A novel 34-kd protein co-isolated with the IgM molecule in surface IgM-expressing cells. Embo J 7:3451-3456.

38. Hermanson, G.G., D. Eisenberg, P.W. Kincade, and R. Wall. 1988. B29: a member of the immunoglobulin gene superfamily exclusively expressed on betalineage cells. Proc Natl Acad Sci U S A 85:6890-6894.

39. Hombach, J., T. Tsubata, L. Leclercq, H. Stappert, and M. Reth. 1990. Molecular components of the B-cell antigen receptor complex of the IgM class. Nature 343:760-762.

40. Matsuuchi, L., M.R. Gold, A. Travis, R. Grosschedl, A.L. DeFranco, and R.B. Kelly. 1992. The membrane IgM-associated proteins MB-1 and Ig-beta are sufficient to promote surface expression of a partially functional B-cell antigen receptor in a nonlymphoid cell line. Proc Natl Acad Sci U S A 89:3404-3408.

41. Burkhardt, A.L., M. Brunswick, J.B. Bolen, and J.J. Mond. 1991. Antiimmunoglobulin stimulation of B lymphocytes activates src-related proteintyrosine kinases. Proc Natl Acad Sci U S A 88:7410-7414.

42. Kurosaki, T., M. Takata, Y. Yamanashi, T. Inazu, T. Taniguchi, T. Yamamoto, and H. Yamamura. 1994. Syk activation by the Src-family tyrosine kinase in the B cell receptor signaling. J Exp Med 179:1725-1729.

43. Minegishi, Y., E. Coustan-Smith, L. Rapalus, F. Ersoy, D. Campana, and M.E. Conley. 1999. Mutations in Igalpha (CD79a) result in a complete block in B-cell development. J Clin Invest 104:1115-1121.

44. Kitamura, D., A. Kudo, S. Schaal, W. Muller, F. Melchers, and K. Rajewsky. 1992. A critical role of lambda 5 protein in B cell development. Cell 69:823-831.

45. Novobrantseva, T.I., V.M. Martin, R. Pelanda, W. Muller, K. Rajewsky, and A. Ehlich. 1999. Rearrangement and expression of immunoglobulin light chain genes can precede heavy chain expression during normal B cell development in mice. $J$ Exp Med 189:75-88. 
46. Cheng, A.M., B. Rowley, W. Pao, A. Hayday, J.B. Bolen, and T. Pawson. 1995. Syk tyrosine kinase required for mouse viability and B-cell development. Nature 378:303-306.

47. Pappu, R., A.M. Cheng, B. Li, Q. Gong, C. Chiu, N. Griffin, M. White, B.P. Sleckman, and A.C. Chan. 1999. Requirement for B cell linker protein (BLNK) in B cell development. Science 286:1949-1954.

48. Fruman, D.A., S.B. Snapper, C.M. Yballe, L. Davidson, J.Y. Yu, F.W. Alt, and L.C. Cantley. 1999. Impaired B cell development and proliferation in absence of phosphoinositide 3-kinase p85alpha. Science 283:393-397.

49. Wienands, J., O. Larbolette, and M. Reth. 1996. Evidence for a preformed transducer complex organized by the B cell antigen receptor. Proc Natl Acad Sci US A 93:7865-7870.

50. Corcoran, A.E. 2005. Immunoglobulin locus silencing and allelic exclusion. Semin Immunol 17:141-154.

51. Roldan, E., M. Fuxa, W. Chong, D. Martinez, M. Novatchkova, M. Busslinger, and J.A. Skok. 2005. Locus 'decontraction' and centromeric recruitment contribute to allelic exclusion of the immunoglobulin heavy-chain gene. Nat Immunol 6:3141.

52. Chowdhury, D., and R. Sen. 2003. Transient IL-7/IL-7R signaling provides a mechanism for feedback inhibition of immunoglobulin heavy chain gene rearrangements. Immunity 18:229-241.

53. Krishnan, M.R., N.T. Jou, and T.N. Marion. 1996. Correlation between the amino acid position of arginine in VH-CDR3 and specificity for native DNA among autoimmune antibodies. J Immunol 157:2430-2439.

54. Reth, M., E. Petrac, P. Wiese, L. Lobel, and F.W. Alt. 1987. Activation of V kappa gene rearrangement in pre-B cells follows the expression of membranebound immunoglobulin heavy chains. Embo J 6:3299-3305.

55. Lewis, S., N. Rosenberg, F. Alt, and D. Baltimore. 1982. Continuing kappa-gene rearrangement in a cell line transformed by Abelson murine leukemia virus. Cell 30:807-816.

56. Hieter, P.A., S.J. Korsmeyer, T.A. Waldmann, and P. Leder. 1981. Human immunoglobulin kappa light-chain genes are deleted or rearranged in lambdaproducing B cells. Nature 290:368-372.

57. Alt, F.W., V. Enea, A.L. Bothwell, and D. Baltimore. 1980. Activity of multiple light chain genes in murine myeloma cells producing a single, functional light chain. Cell 21:1-12.

58. McGuire, K.L., and E.S. Vitetta. 1981. kappa/lambda Shifts do not occur during maturation of murine B cells. J Immunol 127:1670-1673.

59. Hertz, M., and D. Nemazee. 1997. BCR ligation induces receptor editing in IgM+IgD- bone marrow B cells in vitro. Immunity 6:429-436.

60. Schifferli, J.A., Y.C. Ng, and D.K. Peters. 1986. The role of complement and its receptor in the elimination of immune complexes. N Engl J Med 315:488-495.

61. Ravetch, J.V., and R.A. Clynes. 1998. Divergent roles for Fc receptors and complement in vivo. Annu Rev Immunol 16:421-432.

62. Beale, D., and A. Feinstein. 1976. Structure and function of the constant regions of immunoglobulins. Q Rev Biophys 9:135-180. 
63. Capra, J.D., and J.M. Kehoe. 1975. Hypervariable regions, idiotypy, and the antibody-combining site. Adv Immunol 20:1-40.

64. Davies, D.R., E.A. Padlan, and D.M. Segal. 1975. Three-dimensional structure of immunoglobulins. Annu Rev Biochem 44:639-667.

65. Shimizu, A., N. Takahashi, Y. Yaoita, and T. Honjo. 1982. Organization of the constant-region gene family of the mouse immunoglobulin heavy chain. Cell 28:499-506.

66. Padlan, E.A. 1994. Anatomy of the antibody molecule. Mol Immunol 31:169-217.

67. Saul, F.A., L.M. Amzel, and R.J. Poljak. 1978. Preliminary refinement and structural analysis of the Fab fragment from human immunoglobulin new at $2.0 \mathrm{~A}$ resolution. J Biol Chem 253:585-597.

68. Deisenhofer, J. 1981. Crystallographic refinement and atomic models of a human Fc fragment and its complex with fragment B of protein A from Staphylococcus aureus at 2.9- and 2.8-A resolution. Biochemistry 20:2361-2370.

69. Davis, A.C., K.H. Roux, J. Pursey, and M.J. Shulman. 1989. Intermolecular disulfide bonding in IgM: effects of replacing cysteine residues in the mu heavy chain. Embo J 8:2519-2526.

70. Borsos, T., and H.J. Rapp. 1965. Complement fixation on cell surfaces by 19S and 7S antibodies. Science 150:505-506.

71. Sitia, R., C. Alberini, R. Biassoni, A. Rubartelli, S. DeAmbrosis, and D. Vismara. 1988. The control of membrane and secreted heavy chain biosynthesis varies in different immunoglobulin isotypes produced by a monoclonal B cell lymphoma. Mol Immunol 25:189-197.

72. Calame, K.L. 1985. Mechanisms that regulate immunoglobulin gene expression. Annu Rev Immunol 3:159-195.

73. Sitia, R., M. Neuberger, C. Alberini, P. Bet, A. Fra, C. Valetti, G. Williams, and C. Milstein. 1990. Developmental regulation of IgM secretion: the role of the carboxy-terminal cysteine. Cell 60:781-790.

74. Davis, A.C., C. Collins, and M.J. Shulman. 1989. Differential glycosylation of polymeric and monomeric IgM. Mol Immunol 26:147-152.

75. Brouns, G.S., E. de Vries, and J. Borst. 1995. Assembly and intracellular transport of the human B cell antigen receptor complex. Int Immunol 7:359-368.

76. Schweizer, A., J.A. Fransen, T. Bachi, L. Ginsel, and H.P. Hauri. 1988. Identification, by a monoclonal antibody, of a $53-\mathrm{kD}$ protein associated with a tubulo-vesicular compartment at the cis-side of the Golgi apparatus. J Cell Biol 107:1643-1653.

77. Griffiths, G., and K. Simons. 1986. The trans Golgi network: sorting at the exit site of the Golgi complex. Science 234:438-443.

78. Randall, T.D., J.W. Brewer, and R.B. Corley. 1992. Direct evidence that J chain regulates the polymeric structure of IgM in antibody-secreting B cells. $J$ Biol Chem 267:18002-18007.

79. Frutiger, S., G.J. Hughes, N. Paquet, R. Luthy, and J.C. Jaton. 1992. Disulfide bond assignment in human $\mathrm{J}$ chain and its covalent pairing with immunoglobulin M. Biochemistry 31:12643-12647.

80. Randall, T.D., L.B. King, and R.B. Corley. 1990. The biological effects of IgM hexamer formation. Eur J Immunol 20:1971-1979. 
81. Chuang, P.D., and S.L. Morrison. 1997. Elimination of N-linked glycosylation sites from the human IgA1 constant region: effects on structure and function. $J$ Immunol 158:724-732.

82. Hughey, C.T., J.W. Brewer, A.D. Colosia, W.F. Rosse, and R.B. Corley. 1998. Production of IgM hexamers by normal and autoimmune B cells: implications for the physiologic role of hexameric IgM. J Immunol 161:4091-4097.

83. Lamson, G., and M.E. Koshland. 1984. Changes in J chain and mu chain RNA expression as a function of B cell differentiation. J Exp Med 160:877-892.

84. Webb, C.F., C. Das, S. Eaton, K. Calame, and P.W. Tucker. 1991. Novel proteinDNA interactions associated with increased immunoglobulin transcription in response to antigen plus interleukin-5. Mol Cell Biol 11:5197-5205.

85. Kornfeld, R., and S. Kornfeld. 1985. Assembly of asparagine-linked oligosaccharides. Annu Rev Biochem 54:631-664.

86. Hubbard, S.C., and R.J. Ivatt. 1981. Synthesis and processing of asparaginelinked oligosaccharides. Annu Rev Biochem 50:555-583.

87. Rademacher, T.W., R.B. Parekh, and R.A. Dwek. 1988. Glycobiology. Annu Rev Biochem 57:785-838.

88. Bischoff, J., L. Liscum, and R. Kornfeld. 1986. The use of 1deoxymannojirimycin to evaluate the role of various alpha-mannosidases in oligosaccharide processing in intact cells. J Biol Chem 261:4766-4774.

89. Parodi, A.J. 2000. Role of N-oligosaccharide endoplasmic reticulum processing reactions in glycoprotein folding and degradation. Biochem J 348 Pt 1:1-13.

90. Taylor, S.C., A.D. Ferguson, J.J. Bergeron, and D.Y. Thomas. 2004. The ER protein folding sensor UDP-glucose glycoprotein-glucosyltransferase modifies substrates distant to local changes in glycoprotein conformation. Nat Struct Mol Biol 11:128-134.

91. Williams, D.B. 2006. Beyond lectins: the calnexin/calreticulin chaperone system of the endoplasmic reticulum. J Cell Sci 119:615-623.

92. Frickel, E.M., R. Riek, I. Jelesarov, A. Helenius, K. Wuthrich, and L. Ellgaard. 2002. TROSY-NMR reveals interaction between ERp57 and the tip of the calreticulin P-domain. Proc Natl Acad Sci U S A 99:1954-1959.

93. Hosokawa, N., I. Wada, Y. Natsuka, and K. Nagata. 2006. EDEM accelerates ERAD by preventing aberrant dimer formation of misfolded alphal-antitrypsin. Genes Cells 11:465-476.

94. Helenius, A., and M. Aebi. 2001. Intracellular functions of N-linked glycans. Science 291:2364-2369.

95. Flynn, G.C., J. Pohl, M.T. Flocco, and J.E. Rothman. 1991. Peptide-binding specificity of the molecular chaperone BiP. Nature 353:726-730.

96. Gething, M.J. 1999. Role and regulation of the ER chaperone BiP. Semin Cell Dev Biol 10:465-472.

97. Shen, Y., and L.M. Hendershot. 2005. ERdj3, a stress-inducible endoplasmic reticulum DnaJ homologue, serves as a cofactor for BiP's interactions with unfolded substrates. Mol Biol Cell 16:40-50.

98. Forster, M.L., K. Sivick, Y.N. Park, P. Arvan, W.I. Lencer, and B. Tsai. 2006. Protein disulfide isomerase-like proteins play opposing roles during retrotranslocation. J Cell Biol 173:853-859. 
99. Anelli, T., S. Ceppi, L. Bergamelli, M. Cortini, S. Masciarelli, C. Valetti, and R. Sitia. 2007. Sequential steps and checkpoints in the early exocytic compartment during secretory IgM biogenesis. Embo J 26:4177-4188.

100. Mattioli, L., T. Anelli, C. Fagioli, C. Tacchetti, R. Sitia, and C. Valetti. 2006. ER storage diseases: a role for ERGIC-53 in controlling the formation and shape of Russell bodies. J Cell Sci 119:2532-2541.

101. Kozutsumi, Y., M. Segal, K. Normington, M.J. Gething, and J. Sambrook. 1988. The presence of malfolded proteins in the endoplasmic reticulum signals the induction of glucose-regulated proteins. Nature 332:462-464.

102. Ellgaard, L., M. Molinari, and A. Helenius. 1999. Setting the standards: quality control in the secretory pathway. Science 286:1882-1888.

103. Ron, D., and P. Walter. 2007. Signal integration in the endoplasmic reticulum unfolded protein response. Nat Rev Mol Cell Biol 8:519-529.

104. Hirao, K., Y. Natsuka, T. Tamura, I. Wada, D. Morito, S. Natsuka, P. Romero, B. Sleno, L.O. Tremblay, A. Herscovics, K. Nagata, and N. Hosokawa. 2006.

EDEM3, a soluble EDEM homolog, enhances glycoprotein endoplasmic reticulum-associated degradation and mannose trimming. J Biol Chem 281:96509658.

105. Hebert, D.N., and M. Molinari. 2007. In and out of the ER: protein folding, quality control, degradation, and related human diseases. Physiol Rev 87:13771408.

106. Valetti, C., C.E. Grossi, C. Milstein, and R. Sitia. 1991. Russell bodies: a general response of secretory cells to synthesis of a mutant immunoglobulin which can neither exit from, nor be degraded in, the endoplasmic reticulum. J Cell Biol 115:983-994.

107. Wardemann, H., S. Yurasov, A. Schaefer, J.W. Young, E. Meffre, and M.C. Nussenzweig. 2003. Predominant autoantibody production by early human B cell precursors. Science 301:1374-1377.

108. Nemazee, D., and K. Buerki. 1989. Clonal deletion of autoreactive B lymphocytes in bone marrow chimeras. Proc Natl Acad Sci U S A 86:8039-8043.

109. Nemazee, D.A., and K. Burki. 1989. Clonal deletion of B lymphocytes in a transgenic mouse bearing anti-MHC class I antibody genes. Nature 337:562-566.

110. Hartley, S.B., J. Crosbie, R. Brink, A.B. Kantor, A. Basten, and C.C. Goodnow. 1991. Elimination from peripheral lymphoid tissues of self-reactive B lymphocytes recognizing membrane-bound antigens. Nature 353:765-769.

111. Hartley, S.B., M.P. Cooke, D.A. Fulcher, A.W. Harris, S. Cory, A. Basten, and C.C. Goodnow. 1993. Elimination of self-reactive B lymphocytes proceeds in two stages: arrested development and cell death. Cell 72:325-335.

112. Roark, J.H., C.L. Kuntz, K.A. Nguyen, L. Mandik, M. Cattermole, and J. Erikson. 1995. B cell selection and allelic exclusion of an anti-DNA Ig transgene in MRLlpr/lpr mice. J Immunol 154:4444-4455.

113. Erikson, J., M.Z. Radic, S.A. Camper, R.R. Hardy, C. Carmack, and M. Weigert. 1991. Expression of anti-DNA immunoglobulin transgenes in non-autoimmune mice. Nature 349:331-334. 
114. Shlomchik, M.J., A.H. Aucoin, D.S. Pisetsky, and M.G. Weigert. 1987. Structure and function of anti-DNA autoantibodies derived from a single autoimmune mouse. Proc Natl Acad Sci U S A 84:9150-9154.

115. Radic, M.Z., J. Mackle, J. Erikson, C. Mol, W.F. Anderson, and M. Weigert. 1993. Residues that mediate DNA binding of autoimmune antibodies. J Immunol 150:4966-4977.

116. Li, H., Y. Jiang, E.L. Prak, M. Radic, and M. Weigert. 2001. Editors and editing of anti-DNA receptors. Immunity 15:947-957.

117. Gay, D., T. Saunders, S. Camper, and M. Weigert. 1993. Receptor editing: an approach by autoreactive B cells to escape tolerance. J Exp Med 177:999-1008.

118. Tiegs, S.L., D.M. Russell, and D. Nemazee. 1993. Receptor editing in selfreactive bone marrow B cells. J Exp Med 177:1009-1020.

119. Sekiguchi, D.R., R.A. Eisenberg, and M. Weigert. 2003. Secondary heavy chain rearrangement: a mechanism for generating anti-double-stranded DNA B cells. $J$ Exp Med 197:27-39.

120. Radic, M.Z., M.A. Mascelli, J. Erikson, H. Shan, and M. Weigert. 1991. Ig H and L chain contributions to autoimmune specificities. J Immunol 146:176-182.

121. Ibrahim, S.M., M. Weigert, C. Basu, J. Erikson, and M.Z. Radic. 1995. Light chain contribution to specificity in anti-DNA antibodies. J Immunol 155:32233233.

122. Radic, M.Z., J. Erikson, S. Litwin, and M. Weigert. 1993. B lymphocytes may escape tolerance by revising their antigen receptors. J Exp Med 177:1165-1173.

123. Sandel, P.C., and J.G. Monroe. 1999. Negative selection of immature B cells by receptor editing or deletion is determined by site of antigen encounter. Immunity 10:289-299.

124. Prak, E.L., M. Trounstine, D. Huszar, and M. Weigert. 1994. Light chain editing in kappa-deficient animals: a potential mechanism of B cell tolerance. J Exp Med 180:1805-1815.

125. Chen, C., M.Z. Radic, J. Erikson, S.A. Camper, S. Litwin, R.R. Hardy, and M. Weigert. 1994. Deletion and editing of B cells that express antibodies to DNA. $J$ Immunol 152:1970-1982.

126. Chen, C., Z. Nagy, E.L. Prak, and M. Weigert. 1995. Immunoglobulin heavy chain gene replacement: a mechanism of receptor editing. Immunity 3:747-755.

127. Witsch, E.J., H. Cao, H. Fukuyama, and M. Weigert. 2006. Light chain editing generates polyreactive antibodies in chronic graft-versus-host reaction. $J$ Exp Med 203:1761-1772.

128. Li, Y., Y. Louzoun, and M. Weigert. 2004. Editing anti-DNA B cells by Vlambdax. J Exp Med 199:337-346.

129. Gerdes, T., and M. Wabl. 2004. Autoreactivity and allelic inclusion in a B cell nuclear transfer mouse. Nat Immunol 5:1282-1287.

130. Hochedlinger, K., and R. Jaenisch. 2002. Monoclonal mice generated by nuclear transfer from mature B and T donor cells. Nature 415:1035-1038.

131. Casellas, R., Q. Zhang, N.Y. Zheng, M.D. Mathias, K. Smith, and P.C. Wilson. 2007. Igkappa allelic inclusion is a consequence of receptor editing. $J$ Exp Med 204:153-160. 
132. Azulay-Debby, H., and D. Melamed. 2007. B cell receptor editing in tolerance and autoimmunity. Front Biosci 12:2136-2147.

133. Witsch, E.J., and E. Bettelheim. 2008. Allelic and Isotypic Light Chain Inclusion in Peripheral B Cells from Anti-DNA Antibody Transgenic C57BL/6 and BALB/c Mice. J Immunol 180:3708-3718.

134. Kouskoff, V., G. Lacaud, K. Pape, M. Retter, and D. Nemazee. 2000. B cell receptor expression level determines the fate of developing B lymphocytes: receptor editing versus selection. Proc Natl Acad Sci U S A 97:7435-7439.

135. Lam, K.P., R. Kuhn, and K. Rajewsky. 1997. In vivo ablation of surface immunoglobulin on mature B cells by inducible gene targeting results in rapid cell death. Cell 90:1073-1083.

136. Radic, M.Z., S.M. Ibrahim, J. Rauch, S.A. Camper, and M. Weigert. 1995. Constitutive secretion of transgene-encoded IgG2b autoantibodies leads to symptoms of autoimmune disease. J Immunol 155:3213-3222.

137. Tze, L.E., B.R. Schram, K.P. Lam, K.A. Hogquist, K.L. Hippen, J. Liu, S.A. Shinton, K.L. Otipoby, P.R. Rodine, A.L. Vegoe, M. Kraus, R.R. Hardy, M.S. Schlissel, K. Rajewsky, and T.W. Behrens. 2005. Basal immunoglobulin signaling actively maintains developmental stage in immature B cells. PLoS Biol 3:e82.

138. Shaffer, A.L., and M.S. Schlissel. 1997. A truncated heavy chain protein relieves the requirement for surrogate light chains in early B cell development. J Immunol 159:1265-1275.

139. Lang, J., M. Jackson, L. Teyton, A. Brunmark, K. Kane, and D. Nemazee. 1996. $B$ cells are exquisitely sensitive to central tolerance and receptor editing induced by ultralow affinity, membrane-bound antigen. $J$ Exp Med 184:1685-1697.

140. Gaudin, E., Y. Hao, M.M. Rosado, R. Chaby, R. Girard, and A.A. Freitas. 2004. Positive selection of B cells expressing low densities of self-reactive BCRs. $J$ Exp Med 199:843-853.

141. Loder, F., B. Mutschler, R.J. Ray, C.J. Paige, P. Sideras, R. Torres, M.C. Lamers, and R. Carsetti. 1999. B cell development in the spleen takes place in discrete steps and is determined by the quality of B cell receptor-derived signals. $J$ Exp Med 190:75-89.

142. Bannish, G., E.M. Fuentes-Panana, J.C. Cambier, W.S. Pear, and J.G. Monroe. 2001. Ligand-independent signaling functions for the B lymphocyte antigen receptor and their role in positive selection during B lymphopoiesis. J Exp Med 194:1583-1596.

143. Strohal, R., A. Helmberg, G. Kroemer, and R. Kofler. 1989. Mouse Vk gene classification by nucleic acid sequence similarity. Immunogenetics 30:475-493.

144. Levine, M.H., A.M. Haberman, D.B. Sant'Angelo, L.G. Hannum, M.P. Cancro, C.A. Janeway, Jr., and M.J. Shlomchik. 2000. A B-cell receptor-specific selection step governs immature to mature B cell differentiation. Proc Natl Acad Sci US A 97:2743-2748.

145. Cyster, J.G., J.I. Healy, K. Kishihara, T.W. Mak, M.L. Thomas, and C.C. Goodnow. 1996. Regulation of B-lymphocyte negative and positive selection by tyrosine phosphatase CD45. Nature 381:325-328. 
146. Wang, H., and S.H. Clarke. 2003. Evidence for a ligand-mediated positive selection signal in differentiation to a mature B cell. J Immunol 171:6381-6388.

147. Oliver, A.M., F. Martin, and J.F. Kearney. 1999. IgMhighCD21 high lymphocytes enriched in the splenic marginal zone generate effector cells more rapidly than the bulk of follicular B cells. J Immunol 162:7198-7207.

148. Timens, W., A. Boes, T. Rozeboom-Uiterwijk, and S. Poppema. 1989. Immaturity of the human splenic marginal zone in infancy. Possible contribution to the deficient infant immune response. J Immunol 143:3200-3206.

149. Garcia de Vinuesa, C., P. O'Leary, D.M. Sze, K.M. Toellner, and I.C. MacLennan. 1999. T-independent type 2 antigens induce B cell proliferation in multiple splenic sites, but exponential growth is confined to extrafollicular foci. Eur J Immunol 29:1314-1323.

150. Gunn, K.E., and J.W. Brewer. 2006. Evidence that marginal zone B cells possess an enhanced secretory apparatus and exhibit superior secretory activity. $J$ Immunol 177:3791-3798.

151. Oliver, A.M., F. Martin, G.L. Gartland, R.H. Carter, and J.F. Kearney. 1997. Marginal zone B cells exhibit unique activation, proliferative and immunoglobulin secretory responses. Eur J Immunol 27:2366-2374.

152. Saito, T., S. Chiba, M. Ichikawa, A. Kunisato, T. Asai, K. Shimizu, T. Yamaguchi, G. Yamamoto, S. Seo, K. Kumano, E. Nakagami-Yamaguchi, Y. Hamada, S. Aizawa, and H. Hirai. 2003. Notch2 is preferentially expressed in mature $\mathrm{B}$ cells and indispensable for marginal zone B lineage development. Immunity 18:675-685.

153. Tanigaki, K., H. Han, N. Yamamoto, K. Tashiro, M. Ikegawa, K. Kuroda, A. Suzuki, T. Nakano, and T. Honjo. 2002. Notch-RBP-J signaling is involved in cell fate determination of marginal zone B cells. Nat Immunol 3:443-450.

154. Quong, M.W., A. Martensson, A.W. Langerak, R.R. Rivera, D. Nemazee, and C. Murre. 2004. Receptor editing and marginal zone B cell development are regulated by the helix-loop-helix protein, E2A. J Exp Med 199:1101-1112.

155. Cariappa, A., M. Tang, C. Parng, E. Nebelitskiy, M. Carroll, K. Georgopoulos, and S. Pillai. 2001. The follicular versus marginal zone B lymphocyte cell fate decision is regulated by Aiolos, Btk, and CD21. Immunity 14:603-615.

156. Fruehling, S., and R. Longnecker. 1997. The immunoreceptor tyrosine-based activation motif of Epstein-Barr virus LMP2A is essential for blocking BCRmediated signal transduction. Virology 235:241-251.

157. Miller, C.L., A.L. Burkhardt, J.H. Lee, B. Stealey, R. Longnecker, J.B. Bolen, and E. Kieff. 1995. Integral membrane protein 2 of Epstein-Barr virus regulates reactivation from latency through dominant negative effects on protein-tyrosine kinases. Immunity 2:155-166.

158. Casola, S., K.L. Otipoby, M. Alimzhanov, S. Humme, N. Uyttersprot, J.L. Kutok, M.C. Carroll, and K. Rajewsky. 2004. B cell receptor signal strength determines B cell fate. Nat Immunol 5:317-327.

159. Kanayama, N., M. Cascalho, and H. Ohmori. 2005. Analysis of marginal zone B cell development in the mouse with limited B cell diversity: role of the antigen receptor signals in the recruitment of B cells to the marginal zone. J Immunol 174:1438-1445. 
160. Martin, F., and J.F. Kearney. 2002. Marginal-zone B cells. Nat Rev Immunol 2:323-335.

161. Klein, U., K. Rajewsky, and R. Kuppers. 1998. Human immunoglobulin (Ig) $\mathrm{M}+\mathrm{IgD}+$ peripheral blood B cells expressing the CD27 cell surface antigen carry somatically mutated variable region genes: $\mathrm{CD} 27$ as a general marker for somatically mutated (memory) B cells. $J$ Exp Med 188:1679-1689.

162. Yurasov, S., H. Wardemann, J. Hammersen, M. Tsuiji, E. Meffre, V. Pascual, and M.C. Nussenzweig. 2005. Defective B cell tolerance checkpoints in systemic lupus erythematosus. $J$ Exp Med 201:703-711.

163. Enzler, T., G. Bonizzi, G.J. Silverman, D.C. Otero, G.F. Widhopf, A. AnzelonMills, R.C. Rickert, and M. Karin. 2006. Alternative and classical NF-kappa B signaling retain autoreactive $\mathrm{B}$ cells in the splenic marginal zone and result in lupus-like disease. Immunity 25:403-415.

164. ter Borg, E.J., G. Horst, E.J. Hummel, P.C. Limburg, and C.G. Kallenberg. 1990. Measurement of increases in anti-double-stranded DNA antibody levels as a predictor of disease exacerbation in systemic lupus erythematosus. A long-term, prospective study. Arthritis Rheum 33:634-643.

165. Napirei, M., H. Karsunky, B. Zevnik, H. Stephan, H.G. Mannherz, and T. Moroy. 2000. Features of systemic lupus erythematosus in Dnase1-deficient mice. Nat Genet 25:177-181.

166. Kramers, C., M.N. Hylkema, M.C. van Bruggen, R. van de Lagemaat, H.B. Dijkman, K.J. Assmann, R.J. Smeenk, and J.H. Berden. 1994. Anti-nucleosome antibodies complexed to nucleosomal antigens show anti-DNA reactivity and bind to rat glomerular basement membrane in vivo. J Clin Invest 94:568-577.

167. Pisetsky, D.S., and N. Jiang. 2006. The generation of extracellular DNA in SLE: the role of death and sex. Scand J Immunol 64:200-204.

168. Yasutomo, K., T. Horiuchi, S. Kagami, H. Tsukamoto, C. Hashimura, M. Urushihara, and Y. Kuroda. 2001. Mutation of DNASE1 in people with systemic lupus erythematosus. Nat Genet 28:313-314.

169. von Muhlen, C.A., and E.M. Tan. 1995. Autoantibodies in the diagnosis of systemic rheumatic diseases. Semin Arthritis Rheum 24:323-358.

170. Cocca, B.A., S.N. Seal, P. D'Agnillo, Y.M. Mueller, P.D. Katsikis, J. Rauch, M. Weigert, and M.Z. Radic. 2001. Structural basis for autoantibody recognition of phosphatidylserine-beta 2 glycoprotein I and apoptotic cells. Proc Natl Acad Sci US A 98:13826-13831.

171. van Es, J.H., F.H. Gmelig Meyling, W.R. van de Akker, H. Aanstoot, R.H. Derksen, and T. Logtenberg. 1991. Somatic mutations in the variable regions of a human IgG anti-double-stranded DNA autoantibody suggest a role for antigen in the induction of systemic lupus erythematosus. $J$ Exp Med 173:461-470.

172. Radic, M.Z., and M. Weigert. 1994. Genetic and structural evidence for antigen selection of anti-DNA antibodies. Anпи Rev Immunol 12:487-520.

173. Kyle, R.A., T.M. Therneau, S.V. Rajkumar, D.R. Larson, M.F. Plevak, J.R. Offord, A. Dispenzieri, J.A. Katzmann, and L.J. Melton, 3rd. 2006. Prevalence of monoclonal gammopathy of undetermined significance. N Engl J Med 354:13621369. 
174. Tedder, T.F., S. Sato, J.C. Poe, and M. Fujimoto. 2000. CD19 and CD22 regulate a B lymphocyte signal transduction pathway that contributes to autoimmunity. Keio J Med 49:1-13.

175. Sato, S., N. Ono, D.A. Steeber, D.S. Pisetsky, and T.F. Tedder. 1996. CD19 regulates B lymphocyte signaling thresholds critical for the development of B-1 lineage cells and autoimmunity. J Immunol 157:4371-4378.

176. Asano, N., M. Fujimoto, N. Yazawa, S. Shirasawa, M. Hasegawa, H. Okochi, K. Tamaki, T.F. Tedder, and S. Sato. 2004. B Lymphocyte signaling established by the CD19/CD22 loop regulates autoimmunity in the tight-skin mouse. Am J Pathol 165:641-650.

177. Tedder, T.F., A. Baras, and Y. Xiu. 2006. Fcgamma receptor-dependent effector mechanisms regulate CD19 and CD20 antibody immunotherapies for B lymphocyte malignancies and autoimmunity. Springer Semin Immunopathol 28:351-364.

178. Zhang, J., A.K. Somani, and K.A. Siminovitch. 2000. Roles of the SHP-1 tyrosine phosphatase in the negative regulation of cell signalling. Semin Immunol 12:361378.

179. Dziarski, R. 1988. Autoimmunity: polyclonal activation or antigen induction? Immunol Today 9:340-342.

180. Shlomchik, M., M. Mascelli, H. Shan, M.Z. Radic, D. Pisetsky, A. MarshakRothstein, and M. Weigert. 1990. Anti-DNA antibodies from autoimmune mice arise by clonal expansion and somatic mutation. J Exp Med 171:265-292.

181. Huff, J.P., G. Roos, C.L. Peebles, R. Houghten, K.F. Sullivan, and E.M. Tan. 1990. Insights into native epitopes of proliferating cell nuclear antigen using recombinant DNA protein products. $J$ Exp Med 172:419-429.

182. Luger, K., A.W. Mader, R.K. Richmond, D.F. Sargent, and T.J. Richmond. 1997. Crystal structure of the nucleosome core particle at 2.8 A resolution. Nature 389:251-260.

183. Losman, M.J., T.M. Fasy, K.E. Novick, and M. Monestier. 1993. Relationships among antinuclear antibodies from autoimmune MRL mice reacting with histone H2A-H2B dimers and DNA. Int Immunol 5:513-523.

184. Hardin, J.A., and J.O. Thomas. 1983. Antibodies to histones in systemic lupus erythematosus: localization of prominent autoantigens on histones H1 and H2B. Proc Natl Acad Sci U S A 80:7410-7414.

185. Koutouzov, S., A. Cabrespines, Z. Amoura, H. Chabre, C. Lotton, and J.F. Bach. 1996. Binding of nucleosomes to a cell surface receptor: redistribution and endocytosis in the presence of lupus antibodies. Eur J Immunol 26:472-486.

186. Cabrespines, A., D. Laderach, C. Lebosse, J.F. Bach, and S. Koutouzov. 1998. Isolation and characterization of apoptotic nucleosomes, free and complexed with lupus autoantibody generated during hybridoma B-cell apoptosis. J Autoimmun 11:19-27.

187. Radic, M., T. Marion, and M. Monestier. 2004. Nucleosomes are exposed at the cell surface in apoptosis. J Immunol 172:6692-6700.

188. Hughes, G.R., and R.A. Asherson. 1985. Atypical lupus with special reference to ANA negative lupus and lupus subsets. Adv Nephrol Necker Hosp 14:333-346. 
189. Gavanescu, I., D. Vazquez-Abad, J. McCauley, J.L. Senecal, and S. Doxsey. 1999. Centrosome proteins: a major class of autoantigens in scleroderma. J Clin Immunol 19:166-171.

190. Rodriguez, J.L., C. Gelpi, T.M. Thomson, F.J. Real, and J. Fernandez. 1982. Antigolgi complex autoantibodies in a patient with Sjogren syndrome and lymphoma. Clin Exp Immunol 49:579-586.

191. Renier, G., M.C. Rousselet, F. Carrere, A. Croue, C. Andre, F. Oksman, A. Chevailler, and D. Hurez. 1994. Golgi autoantibodies and autoantigens. $J$ Autoimmun 7:133-143.

192. Gentric, A., M. Blaschek, C. Julien, J. Jouquan, Y. Pennec, J.M. Berthelot, D. Mottier, R. Casburn-Budd, and P. Youinou. 1991. Nonorgan-specific autoantibodies in individuals infected with type 1 human immunodeficiency virus. Clin Immunol Immunopathol 59:487-494.

193. Mayet, W.J., E. Hermann, E. Csernok, A. Knuth, T. Poralla, W.L. Gross, and K.H. Meyer zum Buschenfelde. 1991. A human renal cancer line as a new antigen source for the detection of antibodies to cytoplasmic and nuclear antigens in sera of patients with Wegener's granulomatosis. J Immunol Methods 143:57-68.

194. Huidbuchel, E., M. Blaschek, J.M. Seigneurin, A. Lamour, J.M. Berthelot, and P. Youinou. 1991. Anti-organelle and anti-cytoskeletal autoantibodies in the serum of Epstein-Barr virus-infected patients. Ann Med Interne (Paris) 142:343-346.

195. Funaki, T., T. Fujiwara, H.S. Hong, Y. Misumi, M. Nishioka, and Y. Ikehara. 1996. Identification and characterization of a 230-kDa Golgi-associated protein recognized by autoantibodies from a patient with HBV hepatitis. Cell Struct Funct 21:63-72.

196. Kooy, J., B.H. Toh, and P.A. Gleeson. 1994. Heterogeneity of human anti-Golgi auto-antibodies: reactivity with components from 35 to $260 \mathrm{kDa}$. Immunol Cell Biol 72:123-127.

197. Nozawa, K., M.J. Fritzler, C.A. von Muhlen, and E.K. Chan. 2004. Giantin is the major Golgi autoantigen in human anti-Golgi complex sera. Arthritis Res Ther 6:R95-102.

198. Andrade, L.E., E.K. Chan, C.L. Peebles, and E.M. Tan. 1996. Two major autoantigen-antibody systems of the mitotic spindle apparatus. Arthritis Rheum 39:1643-1653.

199. Konstantinov, K., A. von Mikecz, D. Buchwald, J. Jones, L. Gerace, and E.M. Tan. 1996. Autoantibodies to nuclear envelope antigens in chronic fatigue syndrome. J Clin Invest 98:1888-1896.

200. von Muhlen, C.A., E.K. Chan, C.L. Peebles, H. Imai, K. Kiyosawa, and E.M. Tan. 1995. Non-muscle myosin as target antigen for human autoantibodies in patients with hepatitis C virus-associated chronic liver diseases. Clin Exp Immunol 100:67-74.

201. Chan, E.K., J.C. Hamel, J.P. Buyon, and E.M. Tan. 1991. Molecular definition and sequence motifs of the $52-\mathrm{kD}$ component of human SS-A/Ro autoantigen. $J$ Clin Invest 87:68-76.

202. Chou, C.H., and W.H. Reeves. 1992. Recognition of multiple epitopes in the coiled-coil domain of lamin B by human autoantibodies. Mol Immunol 29:10551064. 
203. Doyle, H.A., and M.J. Mamula. 2001. Post-translational protein modifications in antigen recognition and autoimmunity. Trends Immunol 22:443-449.

204. Baeten, D., I. Peene, A. Union, L. Meheus, M. Sebbag, G. Serre, E.M. Veys, and F. De Keyser. 2001. Specific presence of intracellular citrullinated proteins in rheumatoid arthritis synovium: relevance to antifilaggrin autoantibodies. Arthritis Rheum 44:2255-2262.

205. Utz, P.J., T.J. Gensler, and P. Anderson. 2000. Death, autoantigen modifications, and tolerance. Arthritis Res 2:101-114.

206. Mevorach, D. 1999. The immune response to apoptotic cells. Ann N Y Acad Sci 887:191-198.

207. Cooke, M.S., N. Mistry, C. Wood, K.E. Herbert, and J. Lunec. 1997. Immunogenicity of DNA damaged by reactive oxygen species--implications for anti-DNA antibodies in lupus. Free Radic Biol Med 22:151-159.

208. Rumore, P.M., and C.R. Steinman. 1990. Endogenous circulating DNA in systemic lupus erythematosus. Occurrence as multimeric complexes bound to histone. J Clin Invest 86:69-74.

209. Casciola-Rosen, L., A. Rosen, M. Petri, and M. Schlissel. 1996. Surface blebs on apoptotic cells are sites of enhanced procoagulant activity: implications for coagulation events and antigenic spread in systemic lupus erythematosus. Proc Natl Acad Sci U S A 93:1624-1629.

210. Seitz, H.M., T.D. Camenisch, G. Lemke, H.S. Earp, and G.K. Matsushima. 2007. Macrophages and dendritic cells use different Axl/Mertk/Tyro3 receptors in clearance of apoptotic cells. J Immunol 178:5635-5642.

211. Gallucci, S., M. Lolkema, and P. Matzinger. 1999. Natural adjuvants: endogenous activators of dendritic cells. Nat Med 5:1249-1255.

212. Jacob, C.O., L. Pricop, C. Putterman, M.N. Koss, Y. Liu, M. Kollaros, S.A. Bixler, C.M. Ambrose, M.L. Scott, and W. Stohl. 2006. Paucity of clinical disease despite serological autoimmunity and kidney pathology in lupus-prone New Zealand mixed 2328 mice deficient in BAFF. J Immunol 177:2671-2680.

213. Izui, S., P.H. Lambert, and P.A. Miescher. 1977. Failure to detect circulating DNA--anti-DNA complexes by four radioimmunological methods in patients with systemic lupus erythematosus. Clin Exp Immunol 30:384-392.

214. Woodroffe, A.J., J.M. Nozza, K.D. Muller, S.L. McIntosh, G.M. Hale, and A.R. Clarkson. 1988. Circulating immune complexes in systemic lupus erythematosus: a reappraisal of the solid phase $\mathrm{C} 1 \mathrm{q}$ radioimmunoassay. J Clin Lab Immunol 26:57.

215. Comenzo, R.L. 2007. Current and emerging views and treatments of systemic immunoglobulin light-chain (Al) amyloidosis. Contrib Nephrol 153:195-210.

216. Lin, J., G.S. Markowitz, A.M. Valeri, N. Kambham, W.H. Sherman, G.B. Appel, and V.D. D'Agati. 2001. Renal monoclonal immunoglobulin deposition disease: the disease spectrum. J Am Soc Nephrol 12:1482-1492.

217. Nemazee, D. 2000. Receptor selection in B and T lymphocytes. Annu Rev Immunol 18:19-51.

218. Radic, M.Z., and M. Zouali. 1996. Receptor editing, immune diversification, and self-tolerance. Immunity 5:505-511. 
219. Prak, E.L., and M. Weigert. 1995. Light chain replacement: a new model for antibody gene rearrangement. J Exp Med 182:541-548.

220. Chen, C., Z. Nagy, M.Z. Radic, R.R. Hardy, D. Huszar, S.A. Camper, and M. Weigert. 1995. The site and stage of anti-DNA B-cell deletion. Nature 373:252255.

221. Xu, H., H. Li, E. Suri-Payer, R.R. Hardy, and M. Weigert. 1998. Regulation of anti-DNA B cells in recombination-activating gene-deficient mice. J Exp Med 188:1247-1254.

222. Retter, M.W., and D. Nemazee. 1998. Receptor editing occurs frequently during normal B cell development. J Exp Med 188:1231-1238.

223. Brard, F., M. Shannon, E.L. Prak, S. Litwin, and M. Weigert. 1999. Somatic mutation and light chain rearrangement generate autoimmunity in anti-singlestranded DNA transgenic MRL/lpr mice. J Exp Med 190:691-704.

224. Sekiguchi, D.R., L. Yunk, D. Gary, D. Charan, B. Srivastava, D. Allman, M.G. Weigert, and E.T. Prak. 2006. Development and selection of edited B cells in B6.56R mice. J Immunol 176:6879-6887.

225. Seal, S.N., M. Monestier, and M.Z. Radic. 2000. Diverse roles for the third complementarity determining region of the heavy chain (H3) in the binding of immunoglobulin Fv fragments to DNA, nucleosomes and cardiolipin. Eur $J$ Immunol 30:3432-3440.

226. Neeli, I., M.M. Richardson, S.N. Khan, D. Nicolo, M. Monestier, and M.Z. Radic. 2007. Divergent members of a single autoreactive B cell clone retain specificity for apoptotic blebs. Mol Immunol 44:1924-1931.

227. Li, Y., H. Li, and M. Weigert. 2002. Autoreactive B cells in the marginal zone that express dual receptors. J Exp Med 195:181-188.

228. Doyle, C.M., J. Han, M.G. Weigert, and E.T. Prak. 2006. Consequences of receptor editing at the lambda locus: multireactivity and light chain secretion. Proc Natl Acad Sci U S A 103:11264-11269.

229. Liu, S., M.G. Velez, J. Humann, S. Rowland, F.J. Conrad, R. Halverson, R.M. Torres, and R. Pelanda. 2005. Receptor editing can lead to allelic inclusion and development of $\mathrm{B}$ cells that retain antibodies reacting with high avidity autoantigens. J Immunol 175:5067-5076.

230. Huang, H., J.F. Kearney, M.J. Grusby, C. Benoist, and D. Mathis. 2006. Induction of tolerance in arthritogenic B cells with receptors of differing affinity for selfantigen. Proc Natl Acad Sci U S A 103:3734-3739.

231. Cocca, B.A., A.M. Cline, and M.Z. Radic. 2002. Blebs and apoptotic bodies are B cell autoantigens. J Immunol 169:159-166.

232. Chen, C., H. Li, Q. Tian, M. Beardall, Y. Xu, N. Casanova, and M. Weigert. 2006. Selection of anti-double-stranded DNA B cells in autoimmune MRL-lpr/lpr mice. J Immunol 176:5183-5190.

233. Fukuyama, H., F. Nimmerjahn, and J.V. Ravetch. 2005. The inhibitory Fcgamma receptor modulates autoimmunity by limiting the accumulation of immunoglobulin G+ anti-DNA plasma cells. Nat Immunol 6:99-106.

234. Cocca, B.A., S.N. Seal, and M.Z. Radic. 1999. Tandem affinity tags for the purification of bivalent anti-DNA single-chain Fv expressed in Escherichia coli. Protein Expr Purif 17:290-298. 
235. Mandik-Nayak, L., S. Seo, A. Eaton-Bassiri, D. Allman, R.R. Hardy, and J. Erikson. 2000. Functional consequences of the developmental arrest and follicular exclusion of anti-double-stranded DNA B cells. J Immunol 164:1161-1168.

236. Liu, X., and T. Manser. 2005. Antinuclear antigen B cells that down-regulate surface B cell receptor during development to mature, follicular phenotype do not display features of anergy in vitro. $J$ Immunol 174:4505-4515.

237. Guloglu, F.B., and C.A. Roman. 2006. Precursor B cell receptor signaling activity can be uncoupled from surface expression. J Immunol 176:6862-6872.

238. Mielenz, D., A. Ruschel, C. Vettermann, and H.M. Jack. 2003. Immunoglobulin mu heavy chains do not mediate tyrosine phosphorylation of Ig alpha from the ER-cis-Golgi. J Immunol 171:3091-3101.

239. Ferry, H., M. Jones, D.J. Vaux, I.S. Roberts, and R.J. Cornall. 2003. The cellular location of self-antigen determines the positive and negative selection of autoreactive B cells. J Exp Med 198:1415-1425.

240. Kopito, R.R., and R. Sitia. 2000. Aggresomes and Russell bodies. Symptoms of cellular indigestion? EMBO Rep 1:225-231.

241. Skalet, A.H., J.A. Isler, L.B. King, H.P. Harding, D. Ron, and J.G. Monroe. 2005. Rapid B cell receptor-induced unfolded protein response in nonsecretory B cells correlates with pro- versus antiapoptotic cell fate. J Biol Chem 280:39762-39771.

242. Lopes-Carvalho, T., J. Foote, and J.F. Kearney. 2005. Marginal zone B cells in lymphocyte activation and regulation. Curr Opin Immunol 17:244-250.

243. Brewer, J.W., T.D. Randall, R.M. Parkhouse, and R.B. Corley. 1994. Mechanism and subcellular localization of secretory IgM polymer assembly. $J$ Biol Chem 269:17338-17348.

244. Koshland, M.E. 1985. The coming of age of the immunoglobulin J chain. Annu Rev Immunol 3:425-453.

245. Niles, M.J., L. Matsuuchi, and M.E. Koshland. 1995. Polymer IgM assembly and secretion in lymphoid and nonlymphoid cell lines: evidence that $\mathrm{J}$ chain is required for pentamer IgM synthesis. Proc Natl Acad Sci U S A 92:2884-2888.

246. Wiersma, E.J., and M.J. Shulman. 1995. Assembly of IgM. Role of disulfide bonding and noncovalent interactions. J Immunol 154:5265-5272.

247. Anderson, D.R., P.H. Atkinson, and W.J. Grimes. 1985. Major carbohydrate structures at five glycosylation sites on murine $\operatorname{IgM}$ determined by high resolution 1H-NMR spectroscopy. Arch Biochem Biophys 243:605-618.

248. Hendershot, L., D. Bole, G. Kohler, and J.F. Kearney. 1987. Assembly and secretion of heavy chains that do not associate posttranslationally with immunoglobulin heavy chain-binding protein. J Cell Biol 104:761-767.

249. Hendershot, L.M. 1990. Immunoglobulin heavy chain and binding protein complexes are dissociated in vivo by light chain addition. J Cell Biol 111:829837.

250. Shachar, I., R. Amitay, E. Rabinovich, J. Haimovich, and S. Bar-Nun. 1992. Polymerization of secretory IgM in B lymphocytes is prevented by a prior targeting to a degradation pathway. J Biol Chem 267:24241-24247.

251. Ruddock, L.W., and M. Molinari. 2006. N-glycan processing in ER quality control. J Cell Sci 119:4373-4380. 
252. Ma, Y., and L.M. Hendershot. 2004. ER chaperone functions during normal and stress conditions. J Chem Neuroanat 28:51-65.

253. Schweitzer, P.A., S.E. Taylor, and L.D. Shultz. 1991. Synthesis of abnormal immunoglobulins by hybridomas from autoimmune "viable motheaten" mutant mice. J Cell Biol 114:35-43.

254. Obeng, E.A., L.M. Carlson, D.M. Gutman, W.J. Harrington, Jr., K.P. Lee, and L.H. Boise. 2006. Proteasome inhibitors induce a terminal unfolded protein response in multiple myeloma cells. Blood 107:4907-4916.

255. Kopito, R.R. 2000. Aggresomes, inclusion bodies and protein aggregation. Trends Cell Biol 10:524-530.

256. Khan, S.N., E.J. Witsch, N.G. Goodman, A.K. Panigrahi, C. Chen, Y. Jiang, A.M. Cline, J. Erikson, M. Weigert, E.T. Prak, and M. Radic. 2008. Editing and escape from editing in anti-DNA B cells. Proc Natl Acad Sci U S A 105:3861-3866.

257. Neeli, I., S.N. Khan, and M. Radic. 2008. Histone deimination as a response to inflammatory stimuli in neutrophils. J Immunol 180:1895-1902.

258. Bergman, Y., J. Haimovich, and F. Melchers. 1977. An IgM-producing tumor with biochemical characteristics of a small B lymphocyte. Eur J Immunol 7:574579.

259. Bergman, Y., and J. Haimovich. 1977. Characterization of a carcinogen-induced murine B lymphocyte cell line of C3H/eB origin. Eur J Immunol 7:413-417.

260. Linstedt, A.D., and H.P. Hauri. 1993. Giantin, a novel conserved Golgi membrane protein containing a cytoplasmic domain of at least $350 \mathrm{kDa}$. Mol Biol Cell 4:679693.

261. Yoshimura, S.I., N. Nakamura, F.A. Barr, Y. Misumi, Y. Ikehara, H. Ohno, M. Sakaguchi, and K. Mihara. 2001. Direct targeting of cis-Golgi matrix proteins to the Golgi apparatus. $J$ Cell Sci 114:4105-4115.

262. Nakamura, N., C. Rabouille, R. Watson, T. Nilsson, N. Hui, P. Slusarewicz, T.E. Kreis, and G. Warren. 1995. Characterization of a cis-Golgi matrix protein, GM130. J Cell Biol 131:1715-1726.

263. Sato, K., and A. Nakano. 2007. Mechanisms of COPII vesicle formation and protein sorting. FEBS Lett 581:2076-2082.

264. Presley, J.F., T.H. Ward, A.C. Pfeifer, E.D. Siggia, R.D. Phair, and J. LippincottSchwartz. 2002. Dissection of COPI and Arfl dynamics in vivo and role in Golgi membrane transport. Nature 417:187-193.

265. Guillen, E., C. Abeijon, and C.B. Hirschberg. 1998. Mammalian Golgi apparatus UDP-N-acetylglucosamine transporter: molecular cloning by phenotypic correction of a yeast mutant. Proc Natl Acad Sci U S A 95:7888-7892.

266. Tulsiani, D.R., S.C. Hubbard, P.W. Robbins, and O. Touster. 1982. alpha-DMannosidases of rat liver Golgi membranes. Mannosidase II is the GlcNAcMAN5-cleaving enzyme in glycoprotein biosynthesis and mannosidases Ia and IB are the enzymes converting Man9 precursors to Man5 intermediates. $J$ Biol Chem 257:3660-3668.

267. Moremen, K.W. 2002. Golgi alpha-mannosidase II deficiency in vertebrate systems: implications for asparagine-linked oligosaccharide processing in mammals. Biochim Biophys Acta 1573:225-235. 
268. Dinter, A., and E.G. Berger. 1998. Golgi-disturbing agents. Histochem Cell Biol 109:571-590.

269. Ragnarson, B., L. Bengtsson, and A. Haegerstrand. 1992. Labeling with fluorescent carbocyanine dyes of cultured endothelial and smooth muscle cells by growth in dye-containing medium. Histochemistry 97:329-333.

270. Kehry, M., S. Ewald, R. Douglas, C. Sibley, W. Raschke, D. Fambrough, and L. Hood. 1980. The immunoglobulin mu chains of membrane-bound and secreted IgM molecules differ in their C-terminal segments. Cell 21:393-406.

271. Foy, S.P., and L. Matsuuchi. 2001. Association of B lymphocyte antigen receptor polypeptides with multiple chaperone proteins. Immunol Lett 78:149-160.

272. Meunier, L., Y.K. Usherwood, K.T. Chung, and L.M. Hendershot. 2002. A subset of chaperones and folding enzymes form multiprotein complexes in endoplasmic reticulum to bind nascent proteins. Mol Biol Cell 13:4456-4469.

273. Herscovics, A. 1999. Importance of glycosidases in mammalian glycoprotein biosynthesis. Biochim Biophys Acta 1473:96-107.

274. Maley, F., R.B. Trimble, A.L. Tarentino, and T.H. Plummer, Jr. 1989. Characterization of glycoproteins and their associated oligosaccharides through the use of endoglycosidases. Anal Biochem 180:195-204.

275. Shultz, L.D., D.R. Coman, B.L. Lyons, C.L. Sidman, and S. Taylor. 1987. Development of plasmacytoid cells with Russell bodies in autoimmune "viable motheaten" mice. Am J Pathol 127:38-50.

276. Corboy, M.J., P.J. Thomas, and W.C. Wigley. 2005. Aggresome formation. Methods Mol Biol 301:305-327.

277. Reddy, P., A. Sparvoli, C. Fagioli, G. Fassina, and R. Sitia. 1996. Formation of reversible disulfide bonds with the protein matrix of the endoplasmic reticulum correlates with the retention of unassembled Ig light chains. Embo J 15:20772085.

278. Fra, A.M., C. Fagioli, D. Finazzi, R. Sitia, and C.M. Alberini. 1993. Quality control of ER synthesized proteins: an exposed thiol group as a three-way switch mediating assembly, retention and degradation. Embo $J$ 12:4755-4761.

279. Reddy, P.S., and R.B. Corley. 1998. Assembly, sorting, and exit of oligomeric proteins from the endoplasmic reticulum. Bioessays 20:546-554.

280. Vuillier, F., G. Dumas, C. Magnac, M.C. Prevost, A.I. Lalanne, P. Oppezzo, E. Melanitou, G. Dighiero, and B. Payelle-Brogard. 2005. Lower levels of surface Bcell-receptor expression in chronic lymphocytic leukemia are associated with glycosylation and folding defects of the mu and CD79a chains. Blood 105:29332940.

281. Bouwens, L., P. De Bleser, K. Vanderkerken, B. Geerts, and E. Wisse. 1992. Liver cell heterogeneity: functions of non-parenchymal cells. Enzyme 46:155-168.

282. Koffler, D., P.H. Schur, and H.G. Kunkel. 1967. Immunological studies concerning the nephritis of systemic lupus erythematosus. $J$ Exp Med 126:607624.

283. Raz, E., M. Brezis, E. Rosenmann, and D. Eilat. 1989. Anti-DNA antibodies bind directly to renal antigens and induce kidney dysfunction in the isolated perfused rat kidney. J Immunol 142:3076-3082. 
284. Ehrenstein, M.R., D.R. Katz, M.H. Griffiths, L. Papadaki, T.H. Winkler, J.R. Kalden, and D.A. Isenberg. 1995. Human IgG anti-DNA antibodies deposit in kidneys and induce proteinuria in SCID mice. Kidney Int 48:705-711.

285. Mason, L.J., C.T. Ravirajan, A. Rahman, C. Putterman, and D.A. Isenberg. 2004. Is alpha-actinin a target for pathogenic anti-DNA antibodies in lupus nephritis? Arthritis Rheum 50:866-870.

286. Zhao, Z., E. Weinstein, M. Tuzova, A. Davidson, P. Mundel, P. Marambio, and C. Putterman. 2005. Cross-reactivity of human lupus anti-DNA antibodies with alpha-actinin and nephritogenic potential. Arthritis Rheum 52:522-530.

287. Gallo, G., M. Picken, J. Buxbaum, and B. Frangione. 1989. The spectrum of monoclonal immunoglobulin deposition disease associated with immunocytic dyscrasias. Semin Hematol 26:234-245.

288. Karras, A., L.H. Noel, D. Droz, D. Delansorne, J.P. Saint-Andre, P. Aucouturier, M.A. Alyanakian, J.P. Grunfeld, and P. Lesavre. 2002. Renal involvement in monoclonal (type I) cryoglobulinemia: two cases associated with IgG3 kappa cryoglobulin. Am J Kidney Dis 40:1091-1096.

289. Bridoux, F., V. Hugue, O. Coldefy, J.M. Goujon, M. Bauwens, A. Sechet, J.L. Preud'Homme, and G. Touchard. 2002. Fibrillary glomerulonephritis and immunotactoid (microtubular) glomerulopathy are associated with distinct immunologic features. Kidney Int 62:1764-1775.

290. Papo, T., L. Musset, T. Bardin, B. Bucki, C. Jorgensen, E. Dion, A. Quillard, C. de Gennes, J. Sany, P. Godeau, and J.C. Piette. 1996. Cryocrystalglobulinemia as a cause of systemic vasculopathy and widespread erosive arthropathy. Arthritis Rheum 39:335-340.

291. Ball, N.J., W. Wickert, L.H. Marx, and J.F. Thaell. 1993. Crystalglobulinemia syndrome. A manifestation of multiple myeloma. Cancer 71:1231-1234.

292. Fields, M.L., and J. Erikson. 2003. The regulation of lupus-associated autoantibodies: immunoglobulin transgenic models. Curr Opin Immunol 15:709717.

293. Brink, R., C.C. Goodnow, J. Crosbie, E. Adams, J. Eris, D.Y. Mason, S.B. Hartley, and A. Basten. 1992. Immunoglobulin M and D antigen receptors are both capable of mediating B lymphocyte activation, deletion, or anergy after interaction with specific antigen. $J$ Exp Med 176:991-1005.

294. Chen, C., E.L. Prak, and M. Weigert. 1997. Editing disease-associated autoantibodies. Immunity 6:97-105.

295. Gething, M.J., K. McCammon, and J. Sambrook. 1986. Expression of wild-type and mutant forms of influenza hemagglutinin: the role of folding in intracellular transport. Cell 46:939-950.

296. Dul, J.L., S. Aviel, J. Melnick, and Y. Argon. 1996. Ig light chains are secreted predominantly as monomers. J Immunol 157:2969-2975.

297. Storrie, B., and W. Yang. 1998. Dynamics of the interphase mammalian Golgi complex as revealed through drugs producing reversible Golgi disassembly. Biochim Biophys Acta 1404:127-137.

298. Rabouille, C., N. Hui, F. Hunte, R. Kieckbusch, E.G. Berger, G. Warren, and T. Nilsson. 1995. Mapping the distribution of Golgi enzymes involved in the construction of complex oligosaccharides. J Cell Sci 108 ( Pt 4):1617-1627. 
299. Nozawa, K., C.A. Casiano, J.C. Hamel, C. Molinaro, M.J. Fritzler, and E.K. Chan. 2002. Fragmentation of Golgi complex and Golgi autoantigens during apoptosis and necrosis. Arthritis Res 4:R3.

300. Short, B., A. Haas, and F.A. Barr. 2005. Golgins and GTPases, giving identity and structure to the Golgi apparatus. Biochim Biophys Acta 1744:383-395.

301. Prehm, P. 1984. Hyaluronate is synthesized at plasma membranes. Biochem J 220:597-600.

302. Holmes, M.W., M.T. Bayliss, and H. Muir. 1988. Hyaluronic acid in human articular cartilage. Age-related changes in content and size. Biochem J 250:435441.

303. Hauri, H.P., O. Nufer, L. Breuza, H.B. Tekaya, and L. Liang. 2002. Lectins and protein traffic early in the secretory pathway. Biochem Soc Symp:73-82.

304. Cattaneo, A., and M.S. Neuberger. 1987. Polymeric immunoglobulin M is secreted by transfectants of non-lymphoid cells in the absence of immunoglobulin J chain. Embo J 6:2753-2758.

305. Erlandsson, L., K. Andersson, M. Sigvardsson, N. Lycke, and T. Leanderson. 1998. Mice with an inactivated joining chain locus have perturbed IgM secretion. Eur J Immunol 28:2355-2365.

306. Kaminski, M.S., K. Kitamura, D.G. Maloney, and R. Levy. 1987. Idiotype vaccination against murine $\mathrm{B}$ cell lymphoma. Inhibition of tumor immunity by free idiotype protein. J Immunol 138:1289-1296.

307. Maloney, D.G., M.S. Kaminski, D. Burowski, J. Haimovich, and R. Levy. 1985. Monoclonal anti-idiotype antibodies against the murine B cell lymphoma 38C13: characterization and use as probes for the biology of the tumor in vivo and in vitro. Hybridoma 4:191-209.

308. Cohen, S., J. Haimovich, and N. Hollander. 2003. Anti-idiotype x anti-LFA-1 bispecific antibodies inhibit metastasis of B cell lymphoma. J Immunol 170:26952701.

309. Avin, E., J. Haimovich, and N. Hollander. 2004. Anti-idiotype x anti-CD44 bispecific antibodies inhibit invasion of lymphoid organs by B cell lymphoma. $J$ Immunol 173:4736-4743.

310. Wipke, B.T., Z. Wang, J. Kim, T.J. McCarthy, and P.M. Allen. 2002. Dynamic visualization of a joint-specific autoimmune response through positron emission tomography. Nat Immunol 3:366-372. 


\section{VITA}

Salar Khan was born in Coimbatore, India, on May 20, 1974. He finished his high school in 1991 and joined Madras Veterinary College, where he received a Bachelor of Veterinary Science when he graduated in 1997. He completed his Master of Veterinary Science (Veterinary Microbiology) in 2000. He worked as a Senior Research Fellow at the Veterinary College and Research Institute, Namakkal, India. Salar enrolled in the Department of Molecular Sciences at the University of Tennessee Health Science Center in fall 2002. He graduated from UTHSC with a Ph.D. in Molecular Sciences in May 2008. 